\title{
Synthesis and Use of New Substituted 1,3,5-Hexatrienes in Studying Thermally Induced $6 \pi$-Electrocyclizations
}

\author{
Hans Wolf Sünnemann, ${ }^{[a]}$ Martin G. Banwell, ${ }^{[b]}$ and Armin de Meijere* ${ }^{*[a]}$
}

\author{
Dedicated to Professor Miguel Yus on the occasion of his 60th birthday
}

Keywords: Pericyclic Reactions / Regioselectivity / Palladium catalysis / Cross coupling / Oligocyclic systems

\begin{abstract}
An acyclic, two heterocyclic, and two bicyclic alkenylstannanes, 3, 4a, 4b, 8 and $\mathbf{1 1}$, respectively, were synthesized in yields ranging from 43 to $97 \%$, and each was subjected to a sequence of Stille and Heck couplings with 2-bromocyclohexenyl triflate (13) and alkyl (tert-butyl and methyl) acrylate to furnish seven new $1,3,5$-hexatrienes 19, 20, 21, 22- $t \mathrm{Bu}$, 22-Me, 23 and 43, respectively, in 58-84\% yields. For the alkenylstannanes $\mathbf{4 a}, \mathbf{b}, \mathbf{8}$ and $\mathbf{1 1}$, customized combinations of catalysts had to be used. The Stille-Heck sequence involving 13, 3 and tert-butyl acrylate could be performed in a one-pot mode and proceeded in $75 \%$ yield. The hexatrienes were heated in decalin solutions so as to effect $6 \pi$-electrocyclization. Temperatures and reaction times were optimized individually. The hexatrienes 29, 31 and $\mathbf{3 6}$ gave the bi- and tri-
\end{abstract}

cyclic cyclohexadienes 28, 30 and 34, incorporating allylic alcohol and allyl ether termini, by $6 \pi$-electrocyclization and subsequent $[1,5]$-hydrogen shift, as single products in good yields (85-93\%). In contrast, the hexatrienes 19, 20, 21 and 39 furnished mixtures of the initial electrocyclization products 26, 32, 37 and 40 as well as the products of a subsequent [1,5]-hydrogen shift 27, 33, 38 and 41, respectively. The tricyclic hexatrienes 22- $t \mathrm{Bu}, 22-\mathrm{Me}$ and 23 bearing alkyl (tert-butyl, methyl) acrylate termini also selectively gave the tetracyclic dienes 48- $t \mathrm{Bu}, \mathbf{4 8}-\mathrm{Me}$ and 50 in $71-77 \%$ yields by electrocyclizations and subsequent hydrogen shifts.

( Wiley-VCH Verlag GmbH \& Co. KGaA, 69451 Weinheim, Germany, 2007)

\section{Introduction}

Although the Diels-Alder reaction remains unsurpassed in its scope and versatility as a method for accessing sixmembered carbocycles, ${ }^{[1]}$ the more recently developed thermal $6 \pi$-electrocyclizations of $1,3,5$-hexatrienes ${ }^{[2]}$ have come to complement such [4+2] cycloadditions in terms of functionality in and substitution patterns on the rings. ${ }^{[3]}$ Especially since modern metal-catalyzed cross-coupling methodology $^{[4]}$ has made variously substituted 1,3,5-hexatrienes readily accessible, ${ }^{[3,5]}$ their thermal $6 \pi$-electrocyclizations have become a feasible stereoselective approach to oligosubstituted cyclohexa-1,3-dienes. ${ }^{[3,6]}$ However, when ringannelated cyclohexadienes are formed, the initial products were frequently found to undergo subsequent [1,5]-hydrogen shifts so that mixtures of products were obtained. In order to establish whether this sigmatropic process can be

[a] Institut für Organische und Biomolekulare Chemie der GeorgAugust-Universität Göttingen,

Tammannstrasse 2, 37077 Göttingen, Germany Fax: +49-551-399475

E-mail: Armin.deMeijere@chemie.uni-goettingen.de

[b] Research School of Chemistry, Building 35, Australian National University, Canberra ACT0200, Australia

$\square$ Supporting information for this article is available on the WWW under http://www.eurjoc.org or from the author. retarded or accelerated and the selectivity thus enhanced by a proper choice of substituents, we set out to prepare variously unsymmetrically substituted 1,3,5-hexatrienes using previously established ${ }^{[6 b, 7]}$ Stille-Heck cross-coupling sequences, then undertake various functional group transformations, and study the outcome of the thermal rearrangements of the compounds so formed.

\section{Results and Discussion}

\section{Preparation of Substrates}

For the preparation of a set of diverse 1,3,5-hexatrienes by Stille-Heck cross-coupling sequences, a variety of alkenylstannanes had to be synthesized. The new acyclic (trialkylsilylethenyl)stannane $\mathbf{3}$ was prepared by a rhodium-catalyzed hydrostannylation ${ }^{[8]}$ of (tert-butyldimethylsilyl)acetylene (2). ${ }^{[9]}$ It is noteworthy that at $22{ }^{\circ} \mathrm{C}$ this hydrostannylation led to a 1:1 mixture of 3 and its $(Z)$ isomer as well as a trace of the 1,1-disubstituted isomer. However, when this reaction was carried out at $60^{\circ} \mathrm{C}$, the desired alkene 3 was the main product $(57 \%)$ and accompanied by only traces of the isomers just mentioned (Scheme 1). 


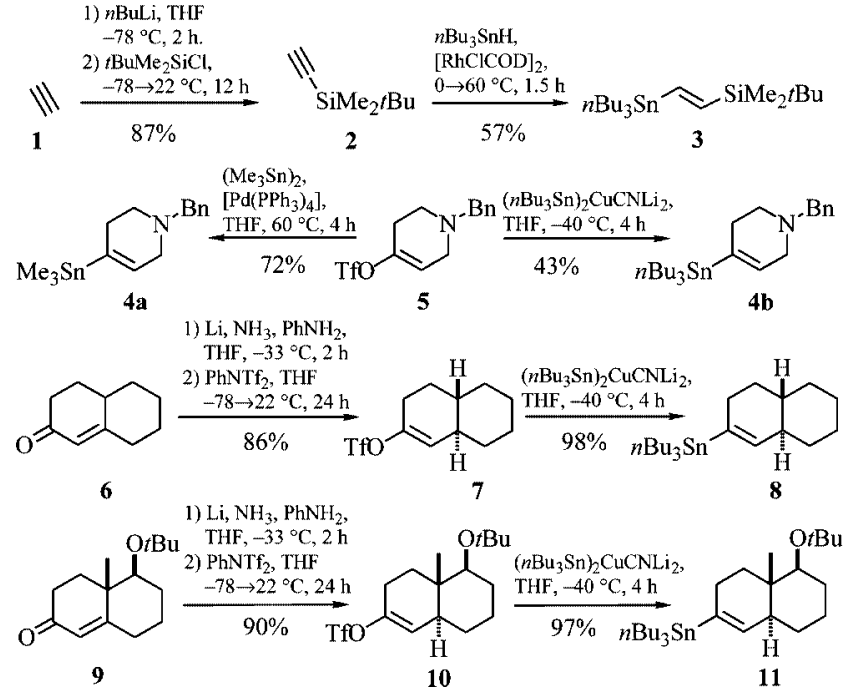

Scheme 1. Synthesis of various alkenylstannanes to be applied in Stille-Heck coupling sequences.

The 4-azacyclohexenylstannanes $\mathbf{4 a , b}$ were synthesized from $N$-benzylpiperidinone via the enol triflate $\mathbf{5}$ which was prepared according to a standard protocol. ${ }^{[10]}$ The tributylstannane $\mathbf{4 b}$ was obtained from $\mathbf{5}$ and dilithium cyanobis(tributylstannyl)cuprate in $43 \%$ yield ${ }^{[11]}$ whereas the corresponding trimethyl(alkenyl)stannane 4a was prepared by a palladium-catalyzed transfer of a trimethyltin group from hexamethylditin to $\mathbf{5}$, and this conversion proceeded with a significantly higher yield of $72 \%$. Because decalin-2ones preferably form the undesired enolate regioisomers, the targeted bicyclo[4.4.0]decenylstannanes $\mathbf{8}$ and $\mathbf{1 1}$ had to be prepared from the $\alpha, \beta$-unsaturated bicyclo[4.4.0]dec-1en-3-ones 6 and 9. Reductive enolate formation using lithium dissolved in liquid ammonia, and trapping with $\mathrm{N}, \mathrm{N}$ bis(trifluoromethylsulfonyl)aniline furnished the trans-annelated bicyclo[4.4.0]dec-2-enol triflates $\mathbf{7}$ and $\mathbf{1 0}$ with complete diastereoselectivity. ${ }^{[12]}$ The latter could be transformed into the corresponding (bicyclodecenyl)tributylstannanes $\mathbf{8}$ and $\mathbf{1 1}$ in 98 and $97 \%$ yields, respectively, by treatment with dilithium cyanobis(tributylstannyl)cuprate. It is noteworthy that the reductive enolization of the corresponding indenone derivatives yielded lithium enolates with the same high diastereoselectivities, but furnished the cis-annelated bicycles (Scheme 1). ${ }^{[\mathrm{a}]}$

2-Tris(n-butylstannyl)-1,4-dioxine (25) was prepared from dioxine and tributyltin chloride according to a literature procedure. ${ }^{[13]}$

The yield associated with the conversion of 2-bromocyclohexanone (12) into 2-bromocyclohexenyl triflate (13) as described previously, ${ }^{[6 b]}$ was significantly improved by employing potassium bis(trimethylsilyl)amide (KHMDS) and trapping the regioselectively formed potassium enolate with trifluoromethanesulfonic anhydride. By such means the target compound 13 was obtained as the sole product in $94 \%$ yield (Scheme 2).

The Stille coupling ${ }^{[14]}$ of compound $\mathbf{1 3}$ with the relevant acyclic (trialkylsilylethenyl)stannane $\mathbf{3}$ was effected using $\left[\mathrm{Pd}\left(\mathrm{PPh}_{3}\right)_{4}\right]$ as catalyst and occurred with a high degree of chemoselectivity for the triflate leaving group to give the intermediate bromodiene $\mathbf{1 4}$ in $80 \%$ yield (Scheme 2). With $\left[\mathrm{Pd}_{2}(\mathrm{dba})_{3}\right]$ in the presence of $\mathrm{LiCl}^{[15]}$ in DMF, the twofold

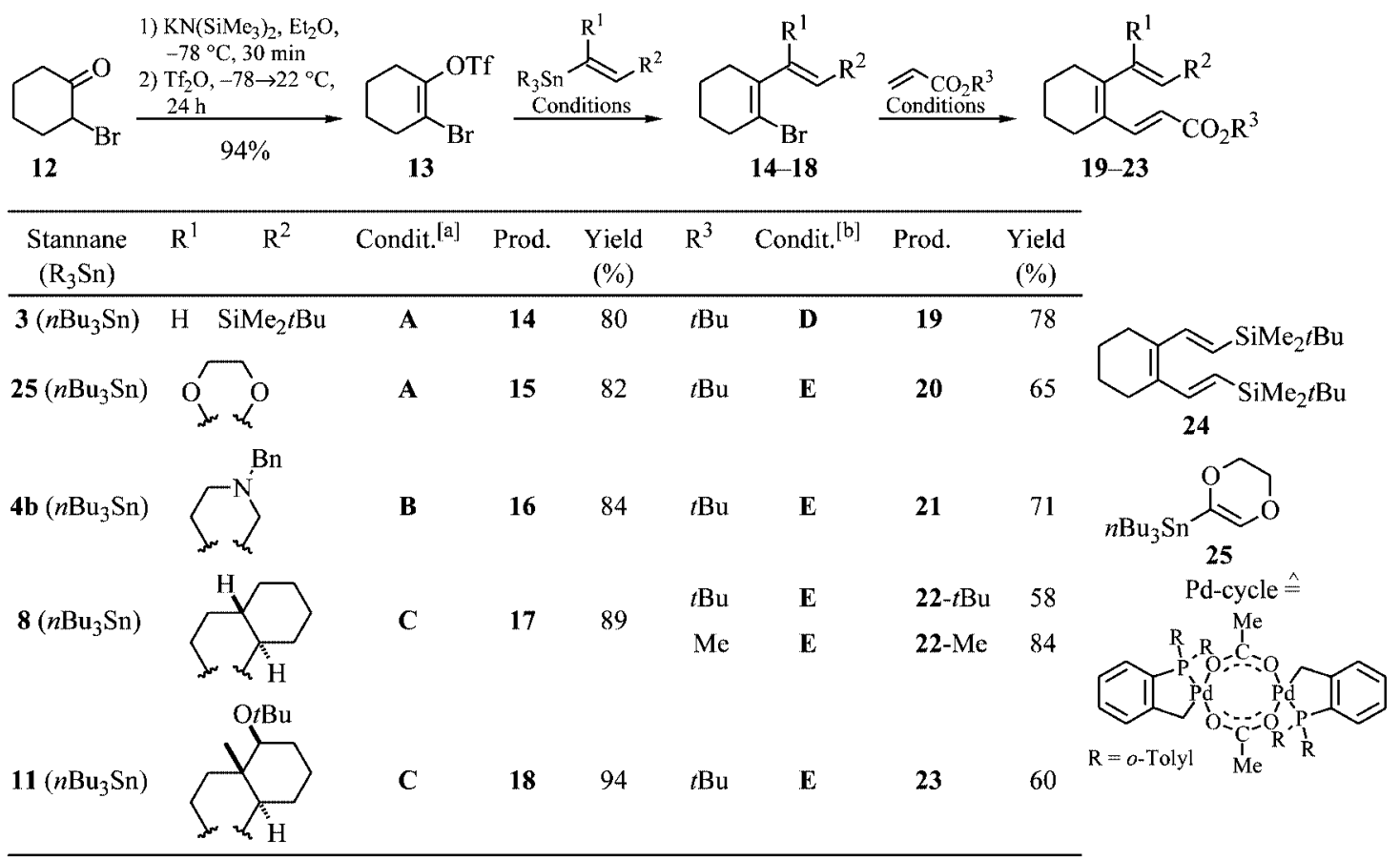

[a] A: [Pd(PPh $)_{4}$ ], LiCl, DMF, $90^{\circ} \mathrm{C}, 10 \mathrm{~h} ; \mathbf{B : ~} \mathrm{Pd}_{2}(\mathrm{dba})_{3}, \mathrm{Cul}, \mathrm{LiCl}, \mathrm{NMP}, 65^{\circ} \mathrm{C}, 5 \mathrm{~h} ; \mathrm{C}^{\circ} \mathrm{Pd}_{2}(\mathrm{dba})_{3}, \mathrm{CuI}, \mathrm{AsPh}, \mathrm{LiCl} ; \mathrm{NMP}, 65^{\circ} \mathrm{C}, 5 \mathrm{~h} .[\mathrm{b}$ ]

D: $\mathrm{Pd}(\mathrm{OAc})_{2}, \mathrm{PPh}_{3}, \mathrm{DMF}, 90^{\circ} \mathrm{C}, 10 \mathrm{~h}$; E: Pd-cycle, $(n \mathrm{Bu})_{4} \mathrm{NOAc}, \mathrm{DMF}, \mathrm{H}_{2} \mathrm{O}, 105^{\circ} \mathrm{C}, 8 \mathrm{~h}$.

Scheme 2. Stille-Heck coupling sequences used to access unsymmetrically 1,6-disubstituted 1,3,5-hexatrienes. 
coupling product $\mathbf{2 4}$ was obtained along with the desired bromodiene 14 in a ratio of $1: 1$. With $\mathrm{CuI}$ and $\mathrm{AsPh}_{3}$ as additives, the catalytic activity towards the reaction with the bromine leaving group was further increased, and the ratio of $\mathbf{2 4 / 1 4}$ increased to $4: 3$.

The subsequent Heck reaction of diene 14 with tert-butyl acrylate was brought about under conventional conditions using $\mathrm{Pd}(\mathrm{OAc})_{2}$ and $\mathrm{PPh}_{3}$ and so provided the unsymmetrically 1,5-disubstituted 1,2-dialkenylcyclohexene 19 in 78\% yield. This Stille-Heck sequence could also be performed in a one-pot procedure to give product 19 in an overall yield of $75 \%$ from 13 which constitutes a real increase in efficiency. Clearly, the rather simple catalytic system derived from $\mathrm{Pd}(\mathrm{OAc})_{2}$ and $\mathrm{PPh}_{3}$ is not retarded by the presence of $\left[\mathrm{Pd}\left(\mathrm{PPh}_{3}\right)_{4}\right]$ and vice versa. As proved by an X-ray structure analysis, the 1,3,5-hexatriene system in $\mathbf{1 9}$ adopts an $s$-trans,s-trans conformation. ${ }^{[16]}$

The dioxinylstannane $\mathbf{2 5}$ was also smoothly coupled with the bromocyclohexenyl triflate 13 using $\left[\mathrm{Pd}\left(\mathrm{PPh}_{3}\right)_{4}\right]$ as a catalyst and so gave the bicyclic bromodiene $\mathbf{1 5}$ in $82 \%$ yield. An attempted Heck coupling with tert-butyl acrylate employing $\mathrm{Pd}(\mathrm{OAc})_{2}$ and $\mathrm{PPh}_{3}$ as a precatalyst gave only low yields $(<20 \%)$ of the bicyclic hexatriene 20 . In bromodienes of type 15, the alkenyl bromide moiety is sterically more encumbered than that associated with compounds of type 14. In order to compensate for the resulting lower reactivity, the palladacycle prepared by heating palladium acetate with tris( $o$-tolyl)phosphane, ${ }^{[17]}$ and a higher temperature were employed for the Heck reaction of $\mathbf{1 5}$ with tertbutyl acrylate. Through such modifications, the 1,3,5-hexatriene 20 was obtained in $65 \%$ yield although full consumption of substrate $\mathbf{1 5}$ required addition of the palladacycle in two portions (Scheme 2). ${ }^{[18]}$

The 4-azacyclohexenylstannane $\mathbf{4 b}$ was not readily coupled with the cyclohexenyl triflate 13 when $\left[\mathrm{Pd}\left(\mathrm{PPh}_{3}\right)_{4}\right]$ was used as catalyst. A much better yield $(84 \%)$ of the bromodiene 16 was achieved with $\left[\mathrm{Pd}_{2}(\mathrm{dba})_{3}\right]$ and $\mathrm{CuI} .^{\left[{ }^{19]}\right.}$ It is noteworthy that the addition of $\mathrm{AsPh}_{3}$ to the catalyst cocktail had no significant influence on the yield. The attempted coupling of the same substrate with the trimethylstannyl derivative $\mathbf{4 a}$ led to low yields regardless of the catalyst. The Heck coupling of compound $\mathbf{1 6}$ with tert-butyl acrylate gave product $\mathbf{2 1}$ in $71 \%$ yield although, once again, the palladacycle had to be added in two portions.

Stille coupling of the bicyclodecenylstannane $\mathbf{8}$ with the bromoenol triflate $\mathbf{1 3}$ was achieved with the precatalyst system consisting of $\left[\mathrm{Pd}_{2}(\mathrm{dba})_{3}\right], \mathrm{AsPh}_{3}$ and $\mathrm{CuI}$ in $\mathrm{DMF}$ and so furnished the tricyclic bromodiene $\mathbf{1 7}$ in 76\% yield. Employing NMP instead of DMF, provided $\mathbf{1 7}$ in $89 \%$ yield, while product 18 was obtained from substrate $\mathbf{1 3}$ and the bicyclodecenylstannane 11 in an even higher yield of $94 \%$.

Heck coupling of the bromodiene 17 with tert-butyl acrylate required two portions of 4 mol- $\%$ each of the palladacycle to furnish the tricyclic hexatriene $22-t \mathrm{Bu}$ in $58 \%$ yield. To achieve reasonable yields, a solvent mixture consisting of MeCN, DMF and water as well as the additive tetrabutylammonium acetate serving as a base proved essential. Under the same conditions, but with two portions of $8 \mathrm{~mol}-$
$\%$ each of the precatalyst, methyl acrylate was coupled with 17 to provide $22-\mathrm{Me}$ in $84 \%$ yield. ${ }^{[20]}$

The functionalized tricyclic bromobutadiene 23, upon Heck reaction with tert-butyl acrylate and with addition of two portions of $4 \mathrm{~mol}-\%$ each of the precatalyst, gave the tricyclic hexatriene 23 in $60 \%$ yield (Scheme 2). ${ }^{[18]}$

Attempts to combine the Stille and Heck coupling reactions so as to prepare the hexatrienes 22- $t \mathrm{Bu}$ and 22-Me directly could not be realized in a one pot procedure, because the Heck reaction under catalysis of the palladacycle did not proceed at all in the presence of NMP.

\section{Studies of the $6 \pi$-Electrocyclization Reactions}

The thermally induced $6 \pi$-electrocyclization reactions were conveniently carried out in the high-boiling, chemically inert solvent decalin. After completion of the reaction, the decalin could easily be removed at ambient temperature under reduced pressure.

For the cyclization of the hexatriene $\mathbf{1 9}$ incorporating an electron-withdrawing tert-butoxycarbonyl substituent, different temperatures were investigated. For example, heating the substrate at $150{ }^{\circ} \mathrm{C}$ for $11 \mathrm{~h}$, afforded a mixture of the two isomeric bicyclo[4.4.0]decadienes 26 and 27, the latter apparently arising from $\mathbf{2 6}$ by a [1,5]-hydrogen shift. However, the major component of the reaction mixture was the starting material 19. At 170 and $190^{\circ} \mathrm{C}$, the transformations were also incomplete, but at $205^{\circ} \mathrm{C}$, the triene 19 was completely consumed within $1 \mathrm{~h}$ and so provided a 1:1.9 mixture of the dienes $\mathbf{2 6}$ and $\mathbf{2 7}$ in a combined yield of $79 \%$. Extended heating of the mixture did not change this ratio significantly. The impossibility readily separating regioisomers $\mathbf{2 6}$ and $\mathbf{2 7}$ detracts from this approach to hexahydronaphthalenes.

In order to be able to extend this investigation to thermal $6 \pi$-electrocyclizations of 1,3,5-hexatrienes with significantly different steric and electronic properties, the tert-butoxycarbonyl moiety in compound $\mathbf{1 9}$ was reduced using diisobutylaluminum hydride (DIBALH) in toluene and thus yielded triene 29 incorporating an allylic alcohol residue that could be protected by its transformation into the tetrahydropyranyl ether 31. ${ }^{[21]}$

Attempts to prepare the allyl ether $\mathbf{3 1}$ directly by Heck cross coupling of the bromodiene $\mathbf{1 4}$ with the relevant partners in the presence of silver acetate or carbonate ${ }^{[22]}$ only provided impure samples of the product and only in low yield $(0-30 \%)$. Under Heck conditions without an added silver salt, a minor amount of the non-conjugated 1,3,6hexatriene was also formed.

Heating triene 31 in decalin at $205^{\circ} \mathrm{C}$ for 45 min cleanly furnished, in 93\% yield, the bicyclic diene 30 resulting from $6 \pi$-electrocyclization and a subsequent $[1,5]$-hydrogen shift (Scheme 3).

After $30 \mathrm{~min}$ at $205^{\circ} \mathrm{C}$, only a mixture of the starting material $\mathbf{3 1}$ and the diene $\mathbf{3 0}$ was observed. Since none of the primary cyclization product analogous to $\mathbf{2 6}$ could be detected, the [1,5]-hydrogen shift obviously proceeds more 


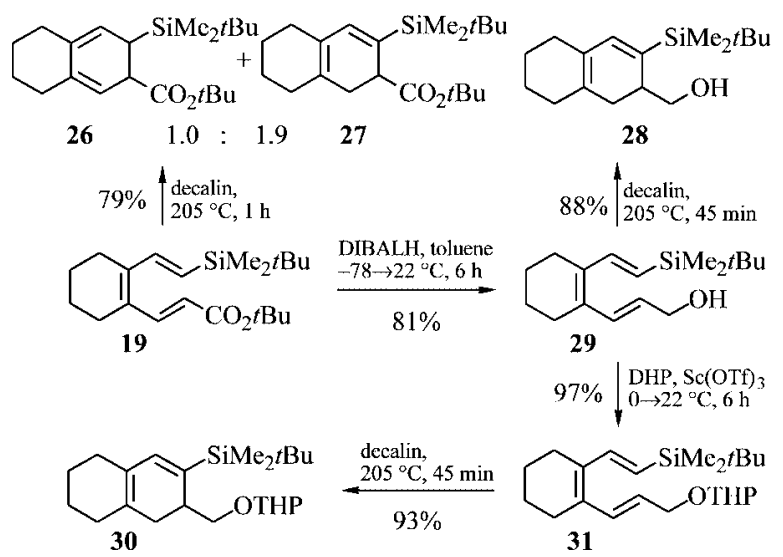

Scheme 3. Thermal $6 \pi$-electrocyclizations of the 1,2-dialkenylcyclohexene derivatives 19, 29 and 31.

rapidly than the $6 \pi$-electrocyclization, a situation that contrasts with the one involving substrate 19 . After $12 \mathrm{~h}$ at $150{ }^{\circ} \mathrm{C}$, no consumption of $\mathbf{3 1}$ was observed. The free allylic alcohol 29 could also be cleanly cyclized to give the diene 28 as a single product in $88 \%$ yield. Thus, this reaction proceeds without decomposition, even at $205^{\circ} \mathrm{C}$.

As observed for the cyclization of the hexatriene 19, upon heating the tert-butoxycarbonyl-substituted hexatriene 20 at $205^{\circ} \mathrm{C}$ for $45 \mathrm{~min}$, a 1:3.1 mixture of the $6 \pi$-electrocyclization product $\mathbf{3 2}$ and isomer $\mathbf{3 3}$ arising from a subsequent [1,5]-hydrogen shift was obtained. In contrast, triene 36 incorporating an allyl ether moiety, which was obtained from compound $\mathbf{2 0}$ by reduction with DIBAL-H ( $85 \%$ yield) and subsequent etherification with $\mathrm{MOMCl}$ (77\%), yielded only the tricyclic diene $\mathbf{3 4}$ in $86 \%$ yield upon heating at $205^{\circ} \mathrm{C}$ for $1 \mathrm{~h}$. Just as observed for congeners 29 and $\mathbf{3 1}$, the $6 \pi$-electrocyclization of compound $\mathbf{3 6}$ is followed by a rapid [1,5]-hydrogen shift (Scheme 4). Interestingly, the hexatriene $\mathbf{3 6}$ could also be transformed into isomer 34 by heating the former at the significantly lower temperature of $150{ }^{\circ} \mathrm{C}$ for $12 \mathrm{~h}$. Indeed, under such conditions the yield of the reaction could even be raised to $91 \%$. The facility with which both the electrocyclization of compound 36 and the subsequent hydrogen migration take place, must arise from the relative electron richness of the substrate although, according to literature precedents, ${ }^{[2 \mathrm{a}]}$ hexatrienes like 19 and 20 carrying electron-withdrawing substituents should cyclize more readily. As with congeners 19 and 20,

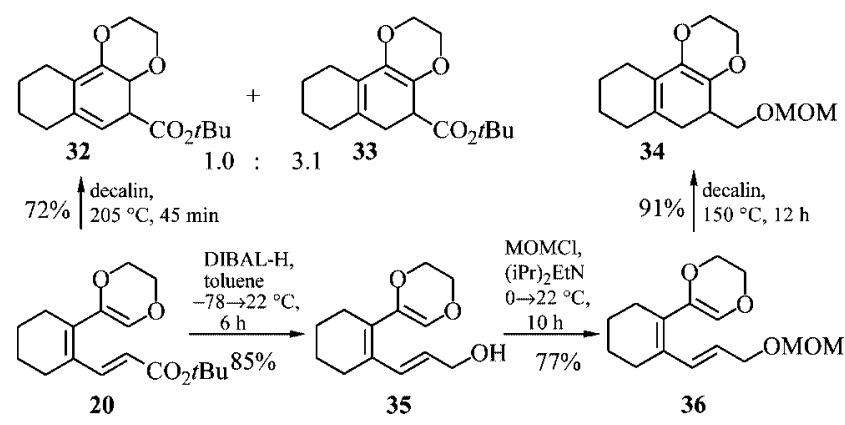

Scheme 4. Thermal $6 \pi$-electrocylizations of the heterobicyclic hexatrienes $\mathbf{2 0}$ and $\mathbf{3 6}$. the heterobicyclic hexatriene $\mathbf{2 1}$ having a tert-butoxycarbonyl substituent required heating at $205^{\circ} \mathrm{C}$ for $45 \mathrm{~min}$ to furnish, in 73\% yield, a 1:2.6 mixture of the dienes 37 and 38. Extended heating did not lead to a significant change in favor of the [1,5]-hydrogen-shifted product $\mathbf{3 8}$.

In contrast, the hexatriene 39 incorporating an allylic alcohol terminus and obtained from precursor 21 in $89 \%$ yield by reduction with DIBALH, upon heating at $205^{\circ} \mathrm{C}$ for 30 min furnished a mixture of the two regioisomeric $\mathbf{4 0}$ and 41 in a ratio of $4.3: 1$ in a total yield of $82 \%$. After $2.5 \mathrm{~h}$ at the same temperature, the ratio of the two regioisomers was $1: 2.5$, although the isomerization of compound $\mathbf{4 0}$ to congener $\mathbf{4 1}$ could not be completed through extended heating, because both products decomposed under such conditions (Scheme 5).

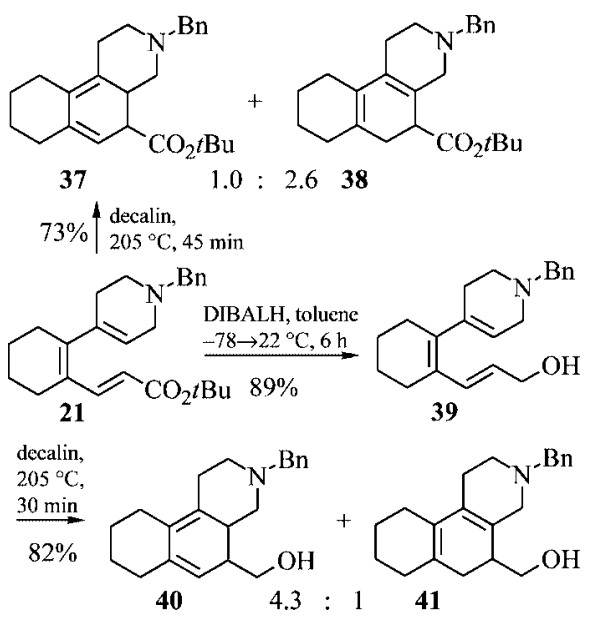

Scheme 5. Thermal $6 \pi$-cyclizations of the heterobicyclic hexatrienes 21 and 39.

The tricyclic 1,3,5-hexatriene $\mathbf{4 3}$ bearing an allylic alcohol group was prepared from $\mathbf{4 2},{ }^{[7 a]}$ as a potential precursor to the steroid framework. Upon heating compound 43 at $205^{\circ} \mathrm{C}$ for $45 \mathrm{~min}$, it reacted to give the regioisomeric tetracyclic compounds $\mathbf{4 4}$ and $\mathbf{4 5}$ in yields of 20 and $26 \%$, respectively. In contrast, the oxohexatriene 46 underwent smooth cyclization and a subsequent (formal) [1,3]-hydrogen shift to yield, exclusively and in $75 \%$ yield, the conjugated steroidal dienone 47 (Scheme 6). ${ }^{[6 \mathrm{c}]}$

The tricyclic hexatrienes $\mathbf{2 2 - t} \mathrm{Bu}, \mathbf{2 2}-\mathrm{Me}$ and particularly 23 may be considered as precursors to Baccharan-type triterpenes. ${ }^{[23]} \mathrm{In}$ decalin solution, $22-t \mathrm{Bu}$ at $205^{\circ} \mathrm{C}$ smoothly rearranged within $45 \mathrm{~min}$ to give $48-t \mathrm{Bu}$ as the sole reaction product and as a single diastereomer in $77 \%$ yield (Scheme 7). Apparently, the initial $6 \pi$-electrocyclization occurred with a high degree of outward-directed disrotational selectivity. ${ }^{[24]}$ Under the same conditions, the tricyclic hexatriene $\mathbf{2 3}$ also gave the tetracycle $\mathbf{5 0}$ as a single product in $71 \%$ yield. By employing the enantiomerically pure and readily available form of bicyclodecenylstannane $\mathbf{1 1}^{[25]}$ in the initial Stille cross coupling, the tetracyclic compound $\mathbf{5 0}$ would also be accessible in enantiomerically pure form.

Interestingly, upon heating the 1-methoxycarbonyl-substituted hexatriene $22-\mathrm{Me}$ at $205^{\circ} \mathrm{C}$ for $30 \mathrm{~min}$, a 7:1 mix- 
<smiles>CCCCOC1CCC2C=C(C3=C(/C=C/C(=O)OCC)CC4(CC3)OCCO4)CCC21C</smiles>

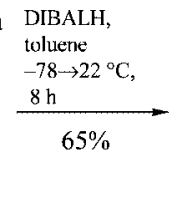<smiles>CCCCC1CCC2C=C(C3=C(/C=C/CO)CC4(CC3)OCCO4)CCC21C</smiles>

43 $46 \% \mid \begin{aligned} & \text { decalin } \\ & 210^{\circ} \mathrm{C}, 45 \mathrm{~min}\end{aligned}$

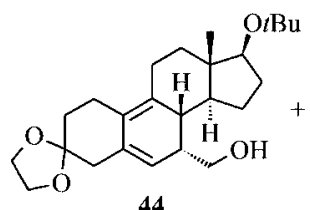<smiles>CCCCC1CCC2C3=C(CCC2(C)C1CO)C1=C(CC3)CC2(CC1)OCCO2</smiles>
$1: 1.3$<smiles>CCCCC1CCC2C=C(C3=C(/C=C/C(=O)OCC)CC(=O)CC3)CCC21C</smiles><smiles>[Z7]C1CC2=CC(=O)CCC2=C2CCC3(CC(=O)OCCCC)C(OCCCC)CCC3C21</smiles>

Scheme 6. Tricyclic 1,3,5-hexatrienes as precursors to steroidal compounds.<smiles>CCCC1=C2CCCC[C@@H]2C=C(C2=C(/C=C/C(=O)OC(C)(C)C)CCCC2)CCC1</smiles>
22- $t \mathrm{Bu}$<smiles>COC(=O)/C=C1\CCCCC1=C1CCCCC2(CCCCC2)CC1</smiles>
22-Me<smiles>CCCCOC1CCCC2(C)C=C(C3=C(/C=C/C(=O)OC(C)(C)C)CCCC3)CCC12C</smiles>
23

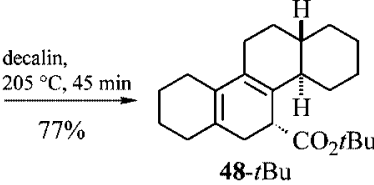

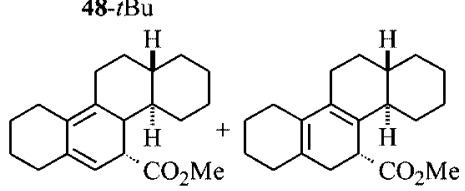

7

48-Me<smiles>CCCCC1=C2CC(C(=O)OCC)C3(C)CCC4=C2C3(CCCC1CCC)C1=C4CCCC1</smiles>

Scheme 7. Thermal rearrangement of the tricyclic hexatrienes 22$t \mathrm{Bu}, \mathbf{2 2}-\mathrm{Me}$ and 23 to tetracycles 48, 49 and $\mathbf{5 0}$.

ture of the initial $6 \pi$-electrocyclization product 49 and the [1,5]-hydrogen-shifted product 48-Me was obtained. In contrast, heating the same reaction mixture for a total of $60 \mathrm{~min}$ provided compound $48-\mathrm{Me}$ exclusively and in a yield of $69 \%$.

\section{Conclusions}

The bicyclo[4.4.0]decadienes $\mathbf{2 8}$ and $\mathbf{3 0}$ can be prepared in an efficient manner through a sequence of Stille-Heck cross couplings and subsequent thermally induced cyclization of the resulting 1,3,5-hexatrienes. They represent interesting intermediates for organic synthesis because they can be employed for the construction of various complex carbon skeletons. ${ }^{[26]}$

The Stille-Heck cross-coupling sequence also provides easy access to various unsymmetrically 1,6-disubstituted 1,3,5-hexatrienes, and further examples could be prepared through straightforward functional-group manipulations. All of those trienes underwent $6 \pi$-electrocyclizations and the primary products of such processes engaged in subsequent $[1,5]$-hydrogen shift reactions, and so provided mixtures of products. However, when the hexatrienes incorporate an allylic alcohol or allyl ether at their terminus, the electrocyclization/[1,5]-hydrogen shift sequences went to completion. Such transformations may find applications in the construction of multifunctional bi- and tricyclic skeletons that can participate in [4+2] cycloaddition reactions and thus resulting in further increase in molecular complexity. ${ }^{[26]}$

\section{Experimental Section}

General Remarks: ${ }^{1} \mathrm{H}$ NMR: Varian VXR-300 (300 MHz), Bruker AM $250(250 \mathrm{MHz})$. Chemical shifts in $\mathrm{CDCl}_{3}$ are reported as $\delta$ values relative to chloroform $(\delta=7.26 \mathrm{ppm})$ or benzene $(\delta=$ $7.20 \mathrm{ppm})$ as internal references. ${ }^{13} \mathrm{C}$ NMR: Varian VXR-300 (75.5 MHz), Bruker AW 250 (62.9 MHz). Chemical shifts in $\mathrm{CDCl}_{3}$ are reported as $\delta$ values relative to chloroform $(\delta=77.0 \mathrm{ppm})$ or benzene $(\delta=128 \mathrm{ppm})$; the multiplicities of the signals were determined by the DEPT (APT) $(62.9 \mathrm{MHz})$ technique and quoted as (+) for $\mathrm{CH}_{3}$ and $\mathrm{CH}$ groups, (-) for $\mathrm{CH}_{2}$ groups and $\left(\mathrm{C}_{\text {quat }}\right)$ for quaternary carbon atoms. IR: Bruker IFS 66. Low-resolution EI mass spectra: Finnigan MAT 95, ionizing voltage $70 \mathrm{eV}$. High-resolution mass spectra: Finnigan MAT 95; preselected ion peak matching at $R$ ca. 10000 to be within \pm 2 ppm of the exact masses. Elemental analyses: Mikroanalytisches Labor des Instituts für Organische und Biomolekulare Chemie der Universität Göttingen, Germany. Melting points are uncorrected. Solvents for extraction and chromatography were of technical grade and distilled before use. Flash chromatography (FC) was performed using Merck Kieselgel 60 (200-400 mesh). Aluminum oxide (ICN Alumina N, Super I) was obtained from ICN Biomedicals. Unless otherwise specified, aluminum oxide was deactivated with $5 \%$ water. TLC analyses were performed using Macherey-Nagel precoated plates, $0.25 \mathrm{~mm}$, Alugram Sil G/UV $\mathrm{UV}_{254}$ (I) and Merck precoated silica gel $60 \mathrm{~F}_{254}$ aluminum sheets (II). All reactions were carried out under dry nitrogen or argon in oven- and/or flame-dried glassware. Unless otherwise specified, solutions of $\mathrm{NH}_{4} \mathrm{Cl}, \mathrm{NaCl}, \mathrm{Na}_{2} \mathrm{SO}_{3}$ and $\mathrm{NaHCO}_{3}$ were saturated aqueous solutions. Benzene, decalin, toluene, THF and diethyl ether were distilled from sodium/benzophenone. Dichloromethane was distilled from $\mathrm{CaH}_{2}$. 1-Benzyl-4-trifluoromethylsulfonyloxy-1,2,3,6-tetrahydropyridine $(\mathbf{5}){ }^{[10]} \Delta^{1,9}$-octalone-2 (6), ${ }^{[27]}$ 5-tert-butoxy-4a-methyl-4,4a,5,6,7,8-hexahydro2(3H)-naphthalenone $(9),{ }^{[25]}$ tributyl(5,6-dihydro[1,4]dioxin-2-yl)stannane (25), ${ }^{[13]} N, N$-bis(trifluoromethylsulfonyl)aniline ${ }^{[28]}$ were prepared according to published procedures.

General Procedure for the Preparation of the Bicycloalkenylstannanes (GP 1): $n$-Butyllithium (2.60 equiv. of a $2.36 \mathrm{~m}$ solution in hexane) was added at $-78{ }^{\circ} \mathrm{C}$ to a solution of diisopropylamine (2.60 equiv.) in THF and the resulting mixture was stirred for $30 \mathrm{~min}$. The resulting solution was treated with tributyltin hydride ( 2.20 equiv.), and stirring was continued for $30 \mathrm{~min}$ before copper(I) cyanide (1.10 equiv.) was added in one portion. The reaction mixture was then warmed to $-50{ }^{\circ} \mathrm{C}$, and after a yellow solution had formed, this was treated with the respective enol triflate (1.00 equiv.) in THF. The resulting solution was warmed to $-25^{\circ} \mathrm{C}$, then stirred continuously at this temperature for $2 \mathrm{~h}$, before being poured into pentane and washed with $\mathrm{NH}_{3}$ solution (10\%), water 
and brine. After drying with $\mathrm{MgSO}_{4}$ and removal of the solvents under reduced pressure, the residue was dissolved in ethyl acetate, and the ensuing solution treated with silver(I) acetate (3.00 equiv.) for $2 \mathrm{~h}$ at ambient temperature, using an unsealed vessel. The reaction mixture was then filtered through Celite, the filtrate washed with water, brine and dried with $\mathrm{MgSO}_{4}$. After removal of the solvent under reduced pressure, the residue was purified by chromatography on neutral aluminum oxide (deactivated with $5 \%$ water).

General Procedure for Stille Couplings of the 2-Bromocyclohex-1enyl Triflate 13 (GP 2): A Pyrex bottle containing a magnetic stirring bar was charged with the specified solvent, the bromoenol triflate $\mathbf{1 3}$ and the specified alkenylstannane. The bottle was sealed with a septum and the solution purged, using inlet and outlet needles, with argon in an ultrasonic bath for $5 \mathrm{~min}$. The specified catalyst mixture was then added, and the resulting suspension was purged with argon for another $5 \mathrm{~min}$. After removal of the septum, the bottle was sealed with a screw cap and then heated with vigorous stirring at the specified temperature for the specified time. The reaction mixture was then poured into diethyl ether and aqueous $\mathrm{NH}_{3}$ solution $(5 \%)$. The organic layer was washed with water, and the combined aqueous layers were extracted with diethyl ether. The combined organic layers were then treated with satd. KF solution, dried with $\mathrm{MgSO}_{4}$, concentrated under reduced pressure, and the residue was purified by chromatography on silica gel.

General Procedure for Heck Reactions of Bromobutadienes with Acrylates (GP 3): A Pyrex bottle containing a magnetic stirring bar was charged with the specified solvent, the bromobutadiene and the acrylate. The bottle was sealed with a septum, and the solution purged, using inlet and outlet needles, with argon in an ultrasonic bath for $5 \mathrm{~min}$. The specified catalyst mixture was then added, and the resulting suspension was purged with argon for another $5 \mathrm{~min}$. After removal of the septum, the bottle was sealed with a screw cap and then heated with vigorous stirring at the specified temperature for the stated time. The reaction mixture was then poured into diethyl ether and water. The organic layer was repeatedly washed with water, and the combined aqueous layers were extracted with diethyl ether. The combined organic layers were then dried with $\mathrm{MgSO}_{4}$, filtered and concentrated under reduced pressure. The residue thus obtained was purified by FC on silica gel.

General Procedure for the Reduction of the tert-ButoxycarbonylSubstituted Hexatrienes to Allylic Alcohols (GP 4): A magnetically stirred solution of the respective $\alpha, \beta$-unsaturated carboxylic ester in toluene at $-78^{\circ} \mathrm{C}$ was treated dropwise with diisobutylaluminum hydride (DIBALH) (4.00-8.00 equiv., $1.00 \mathrm{M}$ in toluene), and the ensuing mixture was stirred at $-78^{\circ} \mathrm{C}$ for $1 \mathrm{~h}$. After warming to ambient temperature, it was stirred for an additional $4 \mathrm{~h}$, then poured into diethyl ether and the mixture washed with $1.00 \mathrm{M}$ $\mathrm{KHSO}_{4}$ solution and water. After extraction of the combined aqueous phases with diethyl ether, washing of the combined organic layers with satd. $\mathrm{NaHCO}_{3}$ solution, water and drying with $\mathrm{MgSO}_{4}$, the volatile components were removed under reduced pressure, and the residue so obtained was subjected to FC.

General Procedure for the Thermally Induced $6 \pi$-Electrocyclization of 1,3,5-Hexatrienes in Solution (GP 5): A thick-walled Pyrex test tube, containing a magnetic stirring bar, was charged with a solution of the respective hexatriene (1.00 equiv.) in the specified solvent. The test tube was sealed with a septum and, using inlet and outlet needles, the solution was purged with argon in an ultrasonic bath for $10 \mathrm{~min}$. The septum was then replaced by a screw cap, and the solution was heated in a prewarmed oil bath at the specified temperature for the stated time. The cooled reaction mixture was concentrated at $25^{\circ} \mathrm{C}$ and $5 \mathrm{mbar}$, and the residue so obtained subjected to FC.

(tert-Butyldimethylsilyl)acetylene (2): A saturated solution of acetylene in THF $(300 \mathrm{~mL})$ was treated carefully and at $-78^{\circ} \mathrm{C}$ over $30 \mathrm{~min}$ with $n \mathrm{BuLi}(66.0 \mathrm{~mL}$ of a $2.38 \mathrm{~m}$ solution in hexane, $156 \mathrm{mmol})$. After stirring the ensuing mixture for $1 \mathrm{~h}$, tert-butylchlorodimethylsilane $(18.1 \mathrm{~g}, 120 \mathrm{mmol})$ was added over $30 \mathrm{~min}$. The reaction mixture was then warmed to ambient temperature and stirred for a total of $24 \mathrm{~h}$. After washing with water $(50 \mathrm{~mL})$, drying with $\mathrm{MgSO}_{4}$, the volatile components were removed by distillation through a $25 \mathrm{~cm}$ Vigreux column. Further distillation of the residue without the column yielded the product 2 (14.6 g, 87\%) as a clear, colorless oil. The analytical data obtained on this material are consistent with those reported previously. ${ }^{[29]}$

(E)-tert-Butyldimethyl(2-tributylstannylvinyl)silane (3): Tributyltin hydride $(4.17 \mathrm{~g}, 14.3 \mathrm{mmol})$ was treated with chlorido(1,5-cyclooctadiene)rhodium(I) dimer $(50.0 \mathrm{mg}, 0.101 \mathrm{mmol})$ at $0{ }^{\circ} \mathrm{C}$ for $5 \mathrm{~min}$. The resulting mixture was treated with (tert-butyldimethylsilyl)acetylene (2) $(2.69 \mathrm{~g}, 10.0 \mathrm{mmol})$, stirred at $60^{\circ} \mathrm{C}$ for $1.5 \mathrm{~h}$, then cooled and subjected directly to FC (70 g of silica gel, pentane) to yield, after concentration of the relevant fractions $\left(R_{\mathrm{f}}=0.8\right)$, the alkenylstannane $3(2.43 \mathrm{~g}, 57 \%)$ as a clear, colorless oil. IR (film): $\tilde{v}=2956 \mathrm{~cm}^{-1}, 2927,2872,2856,1524,1463,1418,1376,1361$, 1340, 1291, 1247, 1197, 1181, 1156, 1110, 1071, 1047, 1008, 958, 938, 873, 862, 825, 773, 748. ${ }^{1} \mathrm{H}$ NMR (300 MHz, $\left.\mathrm{CDCl}_{3}\right): \delta=0.16$ (s, $6 \mathrm{H}, \mathrm{SiCH}_{3}$ ), 0.80-0.97 (m, $14 \mathrm{H}, n \mathrm{Bu} \mathrm{CH} \mathrm{CH}_{3}, n \mathrm{Bu} \mathrm{CH}$ ), 0.99 [s, $\left.9 \mathrm{H}, \mathrm{C}\left(\mathrm{CH}_{3}\right)_{3}\right], 1.20-1.41\left(\mathrm{~m}, 8 \mathrm{H}, n \mathrm{Bu} \mathrm{CH}_{2}\right), 1.42-1.59(\mathrm{~m}, 5 \mathrm{H}$, $n \mathrm{Bu} \mathrm{CH}$ ), $6.58\left(\mathrm{~d},{ }^{3} J=21.6 \mathrm{~Hz}, 1 \mathrm{H}, 2-\mathrm{H}\right), 6.99\left(\mathrm{~d},{ }^{3} J=21.6 \mathrm{~Hz}\right.$, $1 \mathrm{H}, 1-\mathrm{H})$ ppm. ${ }^{13} \mathrm{C} \mathrm{NMR}\left(75.6 \mathrm{MHz}, \mathrm{CDCl}_{3}\right.$, add. APT): $\delta=-6.5$ $\left[+, 2 \mathrm{C}, \mathrm{CH}_{3}, \mathrm{Si}\left(\mathrm{CH}_{3}\right)_{2}\right], 8.8\left[-, \mathrm{C}_{\text {quat }}, C\left(\mathrm{CH}_{3}\right)_{3}\right], 9.5(-, 3 \mathrm{C}, n \mathrm{Bu}$ $\left.\left.\mathrm{CH}_{2}\right), 10.0\left[+, 3 \mathrm{C}, \mathrm{C}\left(\mathrm{CH}_{3}\right)_{3}\right], 13.7(+, 3 \mathrm{C}, n \mathrm{Bu} \mathrm{CH})_{3}\right), 27.4(-, 3$ C, $\left.n \mathrm{Bu} \mathrm{CH}_{2}\right), 29.3\left(-, 3 \mathrm{C}, n \mathrm{Bu} \mathrm{CH}_{2}\right), 151.9(+, \mathrm{CH}), 152.5(+$, $\mathrm{CH}) \mathrm{ppm}$.

1-Benzyl-4-trimethylstannyl-1,2,3,6-tetrahydropyridine (4a): A thick-walled Pyrex bottle, containing a magnetic stirring bar, was charged with a solution of the heterocyclic enol triflate $5(0.840 \mathrm{~g}$, $2.74 \mathrm{mmol})$ in THF $(10 \mathrm{~mL})$, hexamethylditin $(1.00 \mathrm{~g}, 3.05 \mathrm{mmol})$ and lithium chloride $(0.777 \mathrm{~g}, 6.12 \mathrm{mmol})$. This mixture was purged with argon in an ultrasonic bath for $5 \mathrm{~min}$, before tetrakis(triphenylphosphane)palladium(0) $(71.0 \mathrm{mg}, 61.2 \mu \mathrm{mol})$ was added. The bottle was sealed with a screw cap and heated at $60^{\circ} \mathrm{C}$ for $5 \mathrm{~h}$. After cooling to ambient temperature, the reaction mixture was poured into diethyl ether $(100 \mathrm{~mL})$, the mixture washed with water $(2 \times 25 \mathrm{~mL})$, then dried with $\mathrm{MgSO}_{4}$. The volatile components were removed under reduced pressure, and the residue subjected to FC (65 $\mathrm{g}$ of neutral aluminum oxide, 5:1 v/v pentane/diethyl ether elution) to yield, after concentration of the relevant fractions $\left(R_{\mathrm{f}}=\right.$ 0.4), 4a (645 mg, 72\%) as a clear, colorless oil. ${ }^{1} \mathrm{H}$ NMR (300 MHz, $\left.\mathrm{CDCl}_{3}\right): \delta=0.09\left(\mathrm{~s}, 9 \mathrm{H}, \mathrm{SnCH}_{3}\right), 2.24-2.40(\mathrm{~m}, 2 \mathrm{H}, 5-\mathrm{H}), 2.56$ $\left(\mathrm{t},{ }^{3} \mathrm{~J}=5.4 \mathrm{~Hz}, 2 \mathrm{H}, 6-\mathrm{H}\right), 3.02\left(\mathrm{~m}_{\mathrm{C}}, 2 \mathrm{H}, 2-\mathrm{H}\right), 3.58(\mathrm{~s}, 2 \mathrm{H}$, $\left.\mathrm{PhCH}_{2} \mathrm{~N}\right), 5.80\left(\mathrm{~m}_{\mathrm{C}}, 1 \mathrm{H}, 3-\mathrm{H}\right), 7.16-7.47$ (m, $\left.5 \mathrm{H}, \mathrm{Ar}-\mathrm{H}\right) \mathrm{ppm}$.

1-Benzyl-4-tributylstannyl-1,2,3,6-tetrahydropyridine (4b): According to GP 1, a solution of diisopropylamine (1.71 g, $16.9 \mathrm{mmol})$ in THF $(80 \mathrm{~mL}), n \mathrm{BuLi}(10.6 \mathrm{~mL}$ of a $1.60 \mathrm{M}$ solution in hexane, $16.9 \mathrm{mmol})$, tributyltin hydride $(4.17 \mathrm{~g}, 14.3 \mathrm{mmol}), \mathrm{CuCN}$ $(641 \mathrm{mg}, 7.16 \mathrm{mmol})$, the heterocyclic enol triflate $5(2.00 \mathrm{~g}$, $6.51 \mathrm{mmol})$ in THF $(10 \mathrm{~mL})$, after workup with diethyl ether $(100 \mathrm{~mL}), \mathrm{NH}_{3}$ solution $(2 \times 35 \mathrm{~mL})$, water $(2 \times 25 \mathrm{~mL})$, brine $(30 \mathrm{~mL})$, purification with $\mathrm{AgOAc}(6.50 \mathrm{~g}, 39.0 \mathrm{mmol})$ in ethyl acetate $(100 \mathrm{~mL})$, water $(2 \times 30 \mathrm{~mL})$, brine $(25 \mathrm{~mL})$ and $\mathrm{FC}(100 \mathrm{~g}$ on neutral aluminum oxide, $5: 1 \mathrm{v} / \mathrm{v}$ pentane/diethyl ether elution), gave 
4b $(1.29 \mathrm{~g}, 43 \%)$ as a clear, colorless oil. $R_{\mathrm{f}}=0.56$. IR (film): $\tilde{v}=$ $3027 \mathrm{~cm}^{-1}, 2957,2842$, 1604, 1462, 1369, 1336, 1283, 1250, 1142, $1111,1013,945,900,870 .{ }^{1} \mathrm{H}$ NMR $\left(300 \mathrm{MHz}, \mathrm{CDCl}_{3}\right): \delta=0.79$ $1.01\left(\mathrm{~m}, 14 \mathrm{H}, n \mathrm{Bu} \mathrm{CH} \mathrm{CH}_{3}, n \mathrm{Bu} \mathrm{CH}\right.$ ), 1.19-1.39 (m, $8 \mathrm{H}, n \mathrm{Bu} \mathrm{CH}_{2}$ ), 1.40-1.60 (m, $5 \mathrm{H}, n \mathrm{Bu} \mathrm{CH}), 2.31\left(\mathrm{~m}_{\mathrm{C}}, 2 \mathrm{H}, 5-\mathrm{H}\right), 2.52\left(\mathrm{t},{ }^{3} \mathrm{~J}=\right.$ $5.5 \mathrm{~Hz}, 2 \mathrm{H}, 6-\mathrm{H}), 3.00-3.09$ (m, $2 \mathrm{H}, 2-\mathrm{H}), 3.56$ (s, $\left.2 \mathrm{H}, \mathrm{PhCH}_{2} \mathrm{~N}\right)$, $5.76\left(\mathrm{~m}_{\mathrm{C}}, 1 \mathrm{H}, 3-\mathrm{H}\right), 7.24-7.40(\mathrm{~m}, 5 \mathrm{H}, \mathrm{Ar}-\mathrm{H}) \mathrm{ppm} .{ }^{13} \mathrm{C} \mathrm{NMR}$ (75.6 $\mathrm{MHz}, \mathrm{CDCl}_{3}$, additional APT): $\delta=8.9\left(-, 3 \mathrm{C}, n \mathrm{Bu} \mathrm{CH} \mathrm{CH}_{2}\right.$, 13.7 (+, $3 \mathrm{C}, n \mathrm{Bu} \mathrm{CH}$ ), $27.4\left(-, 3 \mathrm{C}, n \mathrm{Bu} \mathrm{CH} \mathrm{CH}_{2}\right), 29.2(-, 3 \mathrm{C}, n \mathrm{Bu}$ $\left.\mathrm{CH}_{2}\right), 32.7\left(-, \mathrm{CH}_{2}\right), 50.2\left(-, \mathrm{CH}_{2}\right), 54.77\left(-, \mathrm{CH}_{2}\right), 63.1\left(-, \mathrm{CH}_{2}\right.$, $\left.\mathrm{PhCH}_{2}\right), 126.9$ (+, $\left.\mathrm{CH}, \mathrm{Ph}\right), 128.1(+, 2 \mathrm{C}, \mathrm{CH}, \mathrm{Ph}), 129.3(+, 2 \mathrm{C}$, $\mathrm{CH}, \mathrm{Ph}), 134.8(+, \mathrm{CH}, \mathrm{C}-3), 138.3\left(-, \mathrm{C}_{\text {quat }}\right), 138.5\left(-, \mathrm{C}_{\text {quat }}\right) \mathrm{ppm}$. MS $(70 \mathrm{eV}): m / z(\%)=465 / 464 / 463 / 462 / 461(1 / 1 / 5 / 1 / 4), 460 / 459 /$ 458/457/456/455/454 (2/2/9/2/8/2/4), 408/407/406/405/404/403/402 (6/8/32/12/26/11/15), 343 (52), 271/270/269/268/267/266/265 (6/3/20/ 5/12/4/6), 251 (6), 224 (28), 174 (9), 172 (84), 134 (6), 91 (100) 65 (6). HRMS: calcd. for $\mathrm{C}_{24} \mathrm{H}_{41} \mathrm{NSn} 456.1839$ (correct HRMS).

2-(trans-3,4,4a,5,6,7,8,8a-Octahydronaphthyl) Trifluoromethanesulfonate (7): Under argon, a mixture of liquid ammonia $(400 \mathrm{~mL})$ and diethyl ether $(200 \mathrm{~mL})$ at $-78{ }^{\circ} \mathrm{C}$ was treated with lithium metal $(322 \mathrm{mg}, 46.4 \mathrm{mmol})$. To the resulting blue solution was added dropwise $\Delta^{1,9}$-octalone-2 $(6)(3.00 \mathrm{~g}, 20.0 \mathrm{mmol})$ and aniline $(133 \mu \mathrm{L}, 1.46 \mathrm{mmol})$ in diethyl ether $(50 \mathrm{~mL})$. The reaction mixture was warmed to $-33^{\circ} \mathrm{C}$ and stirred for $2 \mathrm{~h}$. Excess lithium was oxidized with isoprene, then the mixture was warmed to $22^{\circ} \mathrm{C}$, and the remaining volatile components were removed in vacuo. The residue was dissolved in THF $(250 \mathrm{~mL})$, the solution cooled to $-78^{\circ} \mathrm{C}$, and $N, N$-bis(trifluoromethanesulfonyl)aniline (19.1 g, $53.4 \mathrm{mmol}$ ) in THF $(100 \mathrm{~mL})$ was added dropwise. The reaction mixture was warmed to $22{ }^{\circ} \mathrm{C}$ overnight and stirred for a total of $24 \mathrm{~h}$, it was then directly absorbed on silica gel and subjected to FC ( $400 \mathrm{~g}$ of silica, pentane) to yield, after concentration of the relevant fractions $\left(R_{\mathrm{f}}=0.56\right)$, the product $7(4.87 \mathrm{~g}, 86 \%)$ as a clear colorless oil. IR (film): $\tilde{v}=2926,2857,1684,1448,1418,1369,1248,1208$, 1145, 1081, 1060, 1048, 1024, 998, 963, 886, 854, $615 \mathrm{~cm}^{-1} .{ }^{1} \mathrm{H}$ NMR (250 MHz, $\left.\mathrm{CDCl}_{3}\right): \delta=0.95-1.60(\mathrm{~m}, 6 \mathrm{H}), 1.79\left(\mathrm{~m}_{\mathrm{c}}, 6 \mathrm{H}\right)$, $2.20-2.36\left(\mathrm{dd},{ }^{3} \mathrm{~J}=6.2 \mathrm{~Hz},{ }^{3} \mathrm{~J}=12.0 \mathrm{~Hz}, 1 \mathrm{H}\right), 2.37-2.53(\mathrm{~m}, 1$ $\mathrm{H}), 5.51$ (s, $1 \mathrm{H}, 1-\mathrm{H}) \mathrm{ppm} .{ }^{13} \mathrm{C} \mathrm{NMR}\left(62.9 \mathrm{MHz}, \mathrm{CDCl}_{3}\right.$, add. DEPT): $\delta=26.3\left(-, \mathrm{CH}_{2}\right), 26.9\left(-, \mathrm{CH}_{2}\right), 28.0\left(-, \mathrm{CH}_{2}\right), 29.7$ (-, $\left.\mathrm{CH}_{2}\right), 32.3\left(-, \mathrm{CH}_{2}\right), 32.5\left(-, \mathrm{CH}_{2}\right), 39.7(+, \mathrm{CH}), 40.9(+, \mathrm{CH})$, 117.0 (q, $\left.\mathrm{C}_{\text {quat }},{ }^{1} \mathrm{~J}=340 \mathrm{~Hz}, \mathrm{CF}_{3}\right), 123.1(+, \mathrm{C}-1), 149.0\left(\mathrm{C}_{\mathrm{quat}}, \mathrm{C}-\right.$ 2) $\mathrm{ppm}$. MS $(70 \mathrm{eV}): \mathrm{m} / \mathrm{z}(\%)=284(33)\left[\mathrm{M}^{+}\right], 283(17)\left[\mathrm{M}^{+}-\mathrm{H}\right]$, 151 (100), 133 (47), 119 (5), 95 (18), 91 (31), 69 (44) $\left[\mathrm{CF}_{3}^{+}\right], 55$ (37), 41 (40). $\mathrm{C}_{11} \mathrm{H}_{15} \mathrm{~F}_{3} \mathrm{O}_{3} \mathrm{~S}$ (284.3): calcd. C 46.47, H 5.32; found C 46.27, H 5.38 .

Tributyl(trans-3,4,4a,5,6,7,8,8a-octahydronaphth-2-yl)stannane (8): According to GP 1, a solution of diisopropylamine $(2.92 \mathrm{~mL}$, $20.8 \mathrm{mmol})$ in THF $(140 \mathrm{~mL}), n \mathrm{BuLi}(8.80 \mathrm{~mL}$ of a $2.36 \mathrm{~m}$ solution in hexane, $20.8 \mathrm{mmol})$, tributyltin hydride $(4.75 \mathrm{~mL}, 17.6 \mathrm{mmol})$, $\mathrm{CuCN}(789 \mathrm{mg}, 8.80 \mathrm{mmol})$ and the bicyclic enol triflate $7(2.27 \mathrm{~g}$, $7.95 \mathrm{mmol})$, after workup with pentane $(100 \mathrm{~mL})$ aqueous $\mathrm{NH}_{3}$ solution purification with $\mathrm{AgOAc}(4.00 \mathrm{~g}, 24.0 \mathrm{mmol})$ in ethyl acetate $(140 \mathrm{~mL})$, water $(2 \times 45 \mathrm{~mL})$ and FC $(93 \mathrm{~g}$ on silica gel deactivated with $10 \% \mathrm{NEt}_{3}$, petroleum ether), gave compound 8 (3.31 g, $98 \%$ ) as a clear, colorless oil. $R_{\mathrm{f}}=0.7$. IR (film): $\tilde{v}=2957 \mathrm{~cm}^{-1}$, 2919, 2842, 1604, 1464, 1418, 1376, 1357, 1340, 1286, 1250, 1228, $1193,1148,1110,1071,1023,960,910,875,869,846,688,663$, 595. ${ }^{1} \mathrm{H}$ NMR $\left(250 \mathrm{MHz}, \mathrm{CDCl}_{3}\right): \delta=0.91-1.02(\mathrm{~m}, 15 \mathrm{H}, n \mathrm{Bu}$ $\mathrm{H}), 1.03-1.37(\mathrm{~m}, 4 \mathrm{H}), 1.38-1.51(\mathrm{~m}, 6 \mathrm{H}, n \mathrm{Bu} \mathrm{H}), 1.52-1.83(\mathrm{~m}$, $12 \mathrm{H}), 2.30-2.42(\mathrm{~m}, 2 \mathrm{H}, 3-\mathrm{H}), 5.77\left(\mathrm{~m}_{\mathrm{c}}, 1 \mathrm{H}, 1-\mathrm{H}.\right) \mathrm{ppm} .{ }^{13} \mathrm{C}$ NMR (62.9 MHz, $\mathrm{CDCl}_{3}$, add. DEPT): $\delta=9.2\left(-, \mathrm{CH}_{2}\right), 13.9(+$, $\left.\mathrm{CH}_{3}\right), 26.9\left(-, \mathrm{CH}_{2}\right), 27.1\left(-, \mathrm{CH}_{2}\right) 27.3\left(-, \mathrm{CH}_{2}\right), 27.8\left(-, \mathrm{CH}_{2}\right)$, $29.7\left(-, \mathrm{CH}_{2}\right), 31.4\left(-, \mathrm{CH}_{2}\right), 33.4\left(-, \mathrm{CH}_{2}\right), 33.6\left(-, \mathrm{CH}_{2}\right), 34.1$ $\left(-, \mathrm{CH}_{2}\right), 41.2(+, \mathrm{CH}), 44.8(+, \mathrm{CH}), 139.5\left(\mathrm{C}_{\text {quat }}, \mathrm{C}-2\right), 143.2(+$, $\mathrm{C}-1) \mathrm{ppm}$. MS (70 eV): $m / z(\%)=424(2)\left[\mathrm{M}^{+}\right], 371 / 370 / 369 / 368 /$ $367 / 366 / 365(15 / 19 / 100 / 40 / 79 / 31 / 45)\left[\mathrm{M}^{+}-\mathrm{C}_{4} \mathrm{H}_{9}\right], 315 / 314 / 313 / 312 /$ $311 / 310 / 309(2 / 2 / 18 / 6 / 14 / 6 / 7)\left[\mathrm{M}^{+}-\mathrm{C}_{4} \mathrm{H}_{9}-\mathrm{C}_{4} \mathrm{H}_{8}\right], 292 / 291 / 290 /$ 289/288 (0.5/1/0.5/1/0.5) [ $\left.\mathrm{SnBu}_{3}{ }^{+}\right], 259 / 258 / 257 / 256 / 255 / 254 / 253$ (4/ 2/30/8/25/7/17) $\left[\mathrm{M}^{+}-\mathrm{C}_{4} \mathrm{H}_{9}-2 \times \mathrm{C}_{4} \mathrm{H}_{8}\right], 179 / 178 / 177 / 176 / 175(2 / 1 /$ 4/1/2) $\left[\mathrm{SnBu}^{+}\right], 135(10)\left[\mathrm{M}^{+}-\mathrm{SnBu}_{3}\right], 122 / 120 / 119 / 118 / 117 / 116(1 /$ 5/2/4/2/3) [ $\left.\mathrm{Sn}^{+}\right], 91$ (9), 67 (4), 41 (3). $\mathrm{C}_{22} \mathrm{H}_{42} \mathrm{Sn}$ (424.9): calcd. C 62.13, H 9.96; found C 62.05, H 10.07.

2-(trans-5-tert-Butoxy-4a-methyl-3,4,4a,5,6,7,8,8a-octahydronaphthyl) Trifluoromethanesulfonate (10): Under argon, a mixture of liquid ammonia $(200 \mathrm{~mL})$ and $\mathrm{THF}(150 \mathrm{~mL})$ at $-78^{\circ} \mathrm{C}$ was treated with lithium metal $(233 \mathrm{mg}, 31.2 \mathrm{mmol})$. To the resulting blue solution was added dropwise the $\alpha, \beta$-unsaturated ketone 9 (3.20 g, $13.6 \mathrm{mmol})$ and aniline $(100 \mu \mathrm{L}, 1.10 \mathrm{mmol})$ in THF $(75 \mathrm{~mL})$. The reaction mixture was warmed to $-33{ }^{\circ} \mathrm{C}$ and stirred for $2 \mathrm{~h}$. Excess lithium was oxidized with isoprene, then the mixture was warmed to $22^{\circ} \mathrm{C}$, and the remaining volatile components were removed en vacuo. The residue was dissolved in THF $(150 \mathrm{~mL})$, the solution cooled to $-78{ }^{\circ} \mathrm{C}$, and $N, N$-bis(trifluoromethanesulfonyl)aniline (12.1 g, $32.5 \mathrm{mmol})$ in THF $(100 \mathrm{~mL})$ was added dropwise. The reaction mixture was warmed to $22{ }^{\circ} \mathrm{C}$ overnight and stirred for a total of $24 \mathrm{~h}$, it was then directly absorbed on silica gel and subjected to FC ( $80 \mathrm{~g}$ of silica, 20:1 v/v pentane/diethylether elution) to yield, after concentration of the relevant fractions $\left(R_{\mathrm{f}}=0.43\right)$, the product $10(4.54 \mathrm{~g}, 90 \%)$ as a clear colorless oil. IR (film): $\tilde{v}=$ 2976, 2933, 2867, 1685, 1489, 1417, 1363, 1319, 1247, 1208, 1143, 1091, 1078, 1042, 1008, 991, 958, 929, 871, 816, $764 \mathrm{~cm}^{-1} .{ }^{1} \mathrm{H}$ NMR (250 MHz, $\left.\mathrm{CDCl}_{3}\right): \delta=0.73\left(\mathrm{~s}, 3 \mathrm{H}, \mathrm{CH}_{3}\right), 0.80-0.95(\mathrm{~m}, 3$ $\mathrm{H}), 1.02\left[\mathrm{~s}, 9 \mathrm{H}, \mathrm{C}\left(\mathrm{CH}_{3}\right)_{3}\right], 1.33-1.63(\mathrm{~m}, 5 \mathrm{H}), 1.77\left(\mathrm{dd},{ }^{3} \mathrm{~J}=\right.$ $\left.5.0 \mathrm{~Hz},{ }^{3} \mathrm{~J}=11.3 \mathrm{~Hz}, 1 \mathrm{H}, 8 \mathrm{a}-\mathrm{H}\right) 1.91-2.23(\mathrm{~m}, 2 \mathrm{H}), 2.69\left(\mathrm{dd},{ }^{3} J\right.$ $\left.=5.9 \mathrm{~Hz},{ }^{3} \mathrm{~J}=8.8 \mathrm{~Hz}, 1 \mathrm{H}, 5-\mathrm{H}\right), 5.18(\mathrm{~s}, 1 \mathrm{H}, 1 \mathrm{H}) .{ }^{13} \mathrm{C} \mathrm{NMR}$ (62.9 MHz, $\mathrm{C}_{6} \mathrm{D}_{6}$, add. DEPT): $\delta=10.2\left(+, \mathrm{CH}_{3}\right), 24.6\left(-, \mathrm{CH}_{2}\right)$, $25.2\left(-, \mathrm{CH}_{2}\right), 26.4\left(-, \mathrm{CH}_{2}\right), 28.9\left[+, 3 \mathrm{C},\left(\mathrm{C}_{\left.\left(\mathrm{CH}_{3}\right)_{3}\right],} 30.3\left(-, \mathrm{CH}_{2}\right)\right.\right.$, $34.1\left(-, \mathrm{CH}_{2}\right), 37.5\left(\mathrm{C}_{\text {quat }}, \mathrm{C}-4 \mathrm{a}\right), 43.2(+, \mathrm{C}-8 \mathrm{a}), 73.0(+, \mathrm{C}-5), 77.2$ $\left[\mathrm{C}_{\text {quat }}, C\left(\mathrm{CH}_{3}\right)_{3}\right], 121.3(+, \mathrm{CH}, \mathrm{C}-1), 122.9\left(\mathrm{q}, \mathrm{C}_{\text {quat }},{ }^{1} J=315 \mathrm{~Hz}\right.$, $\left.\mathrm{CF}_{3}\right), 148.4\left(\mathrm{C}_{\text {quat }}, \mathrm{C}-2\right)$. MS $(70 \mathrm{eV}): m / z(\%)=370(2)\left[\mathrm{M}^{+}\right], 313$ (40) $\left[\mathrm{M}^{+}-\mathrm{C}_{4} \mathrm{H}_{9}\right], 295$ (21), 269 (4), 229 (3), 181 (100), 163 (22), 121 (8), 111 (3), 57 (67) $\left[\mathrm{C}_{4} \mathrm{H}_{9}{ }^{+}\right] \cdot \mathrm{C}_{16} \mathrm{H}_{25} \mathrm{~F}_{3} \mathrm{O}_{4} \mathrm{~S}$ (370.4): calcd. $\mathrm{C}$ 51.88, H 6.80; found C 51.61, H 6.57 .

(5-tert-Butoxy-4a-methyl-3,4,5,6,7,8,8a-heptahydronaphthyl)tributylstannane (11): According to GP 1, a solution of diisopropylamine $(3.66 \mathrm{~mL}, 26.0 \mathrm{mmol})$ in THF $(150 \mathrm{~mL}), n B u L i(11.0 \mathrm{~mL}$, $26.0 \mathrm{mmol}, 2.36 \mathrm{M})$, tributyltin hydride $(5.93 \mathrm{~mL}, 22.1 \mathrm{mmol})$, CuCN (986 mg, $11.0 \mathrm{mmol}$ ) and the triflate 10 (3.70 g, $10.0 \mathrm{mmol})$, after workup with pentane $(150 \mathrm{~mL})$, aqueous $\mathrm{NH}_{3}$ solution $(3 \times 40 \mathrm{~mL})$, purification with $\mathrm{AgOAc}(4.99 \mathrm{~g}, 30.0 \mathrm{mmol})$ in ethyl acetate $(150 \mathrm{~mL})$, water $(2 \times 50 \mathrm{~mL})$ and $\mathrm{FC}(134 \mathrm{~g}$ on silica gel deactivated with $10 \% \mathrm{NEt}_{3}$, light petroleum), gave compound $\mathbf{1 1}$ $(4.96 \mathrm{~g}, 97 \%)$ as a clear, colorless oil. $\left(R_{\mathrm{f}}=0.5,20: 1 \mathrm{v} / \mathrm{v}\right.$ light petroleum/diethyl ether). IR (film): $\tilde{v}=2956 \mathrm{~cm}^{-1}, 2926,1685,1464$, $1427,1418,1376,1361,1272,1248,1192,1048,1020,1002,880$, 877, 844, 768. ${ }^{1} \mathrm{H}$ NMR $\left(250 \mathrm{MHz}, \mathrm{C}_{6} \mathrm{D}_{6}\right): \delta=0.78-1.05(\mathrm{~m}, 15$ $\left.\mathrm{H}, n \mathrm{Bu} \mathrm{CH} \mathrm{CH}_{3}, n \mathrm{Bu} \mathrm{CH} \mathrm{CH}_{2}\right), 1.08\left(\mathrm{~s}, 3 \mathrm{H}, \mathrm{CH}_{3}\right), 1.11$ [s, $9 \mathrm{H}, \mathrm{C}-$ $\left.\left(\mathrm{CH}_{3}\right)_{3}\right], 1.20-1.79(\mathrm{~m}, 19 \mathrm{H}), 1.83-1.97(\mathrm{~m}, 1 \mathrm{H}), 2.00-2.12(\mathrm{~m}, 1$ H) $2.30-2.42(\mathrm{~m}, 2 \mathrm{H}, 3-\mathrm{H}), 2.95\left(\mathrm{dd},{ }^{3} \mathrm{~J}=7.0,{ }^{3} \mathrm{~J}=8.4 \mathrm{~Hz}, 1 \mathrm{H}\right.$, 5-H), $5.87\left(\mathrm{~d},{ }^{3} \mathrm{~J}=0.7 \mathrm{~Hz}, 1 \mathrm{H}, 1-\mathrm{H}\right) \mathrm{ppm} .{ }^{13} \mathrm{C} \mathrm{NMR}(62.9 \mathrm{MHz}$, $\mathrm{C}_{6} \mathrm{D}_{6}$, add. DEPT): $\delta=9.2\left(-, n \mathrm{Bu} \mathrm{CH}_{2}\right), 10.8\left(+, \mathrm{CH}_{3}\right), 14.0(+$, $\left.n \mathrm{Bu} \mathrm{CH}_{3}\right), 25.1\left(-, \mathrm{CH}_{2}\right), 27.3\left(-, \mathrm{CH}_{2}\right), 27.8\left(-, n \mathrm{Bu} \mathrm{CH}_{2}\right), 29.2$ $\left[+, 3 \mathrm{C}, \mathrm{C}\left(\mathrm{CH}_{3}\right)_{3}\right], 29.7\left(-, n \mathrm{Bu} \mathrm{CH}_{2}\right), 30.7\left(-, \mathrm{CH}_{2}\right), 31.0\left(-, \mathrm{CH}_{2}\right)$, $35.5\left(-, \mathrm{CH}_{2}\right), 37.8\left(\mathrm{C}_{\text {quat }}, \mathrm{C}-4 \mathrm{a}\right), 46.1(+, \mathrm{C}-8 \mathrm{a}), 72.5\left[\mathrm{C}_{\text {quat }}\right.$, $\left.\mathrm{C}\left(\mathrm{CH}_{3}\right)\right], 77.6(+, \mathrm{C}-5), 139.3\left(\mathrm{C}_{\text {quat }}, \mathrm{C}-2\right), 141.2(+, \mathrm{C}-1) \mathrm{ppm}$. MS $(70 \mathrm{eV}): m / z(\%)=512(1)\left[\mathrm{M}^{+}\right], 457 / 456 / 455 / 454 / 453 / 452 / 451(16 /$ 
24/100/44/83/33/46) $\left[\mathrm{M}^{+}-\mathrm{C}_{4} \mathrm{H}_{9}\right], 401 / 400 / 399 / 398 / 397 / 396 / 395$ (1/ $1 / 10 / 4 / 8 / 4 / 5)\left[\mathrm{M}^{+}-\mathrm{C}_{4} \mathrm{H}_{9}-\mathrm{C}_{4} \mathrm{H}_{8}\right], 345 / 344 / 343 / 342 / 341 / 340 / 339$ (1/ 2/13/3/10/3/4) $\left[\mathrm{M}^{+}-\mathrm{C}_{4} \mathrm{H}_{9}-2 \times \mathrm{C}_{4} \mathrm{H}_{8}\right], 293 / 292 / 291 / 290 / 289 / 288 /$ $287(1 / 1 / 6 / 3 / 5 / 2 / 3)\left[\mathrm{SnBu}_{3}{ }^{+}\right], 237 / 236 / 235 / 234 / 233 / 232 / 231(1 / 1 / 4 / 2 /$ $3 / 2 / 1)\left[\mathrm{SnBu}_{2} \mathrm{H}^{+}\right], 179 / 178 / 177 / 176 / 175(2 / 1 / 4 / 1 / 2)\left[\mathrm{SnBu}^{+}\right], 147(6)$, 105 (5), 57 (18) $\left[\mathrm{Bu}^{+}\right], 41$ (2). $\mathrm{C}_{27} \mathrm{H}_{52} \mathrm{OSn}$ (511.4): calcd. C 63.41, H 10.25; found C 63.44, H 9.98.

I(E)-2-(2-Bromocyclohex-1-enyl)vinyl]tert-butyldimethylsilane (14): According to GP 2, a solution of bromoenol triflate 13 (309 mg, $1.00 \mathrm{mmol})$ in DMF $(10 \mathrm{~mL})$ with the alkenylstannane $3(431 \mathrm{mg}$, $1.00 \mathrm{mmol}$ ), after treatment with tetrakis(triphenylphosphane)palladium $(57.8 \mathrm{mg}, 50.0 \mu \mathrm{mol}), \mathrm{LiCl}(127 \mathrm{mg}, 3.00 \mathrm{mmol})$ at $90^{\circ} \mathrm{C}$ for $12 \mathrm{~h}$, workup with diethyl ether $(50 \mathrm{~mL})$, water $(2 \times 20 \mathrm{~mL})$, extraction with diethyl ether $(2 \times 20 \mathrm{~mL})$, and FC $(55 \mathrm{~g}$ of silica gel, pentane), gave compound $\mathbf{1 4}(240 \mathrm{mg}, 80 \%)$ as a colorless oil. $R_{\mathrm{f}}=0.6$. IR (film): $\tilde{v}=2951 \mathrm{~cm}^{-1}, 2929,2857,2882,1614,1575$, $1470,1462,1448,1435,1409,1389,1361,1334,1247,1193,1137$, $1089,1007,987,973,938,874,832,811,791 .{ }^{1} \mathrm{H}$ NMR $(300 \mathrm{MHz}$, $\left.\mathrm{CDCl}_{3}\right): \delta=0.09\left[\mathrm{~s}, 6 \mathrm{H}, \mathrm{Si}\left(\mathrm{CH}_{3}\right)_{2}\right], 0.89\left[\mathrm{~s}, 9 \mathrm{H}, \mathrm{C}\left(\mathrm{CH}_{3}\right)_{3}\right], 1.72$ $\left[\mathrm{m}_{\mathrm{c}}, 4 \mathrm{H}, 4^{\prime}\left(5^{\prime}\right)-\mathrm{H}\right], 2.29\left(\mathrm{~m}_{\mathrm{C}}, 2 \mathrm{H}, 6^{\prime}-\mathrm{H}\right), 2.64\left(\mathrm{~m}, 2 \mathrm{H}, 3^{\prime}-\mathrm{H}\right), 5.92$ (d, $\left.{ }^{3} J=19.2 \mathrm{~Hz}, 1 \mathrm{H}, 1-\mathrm{H}\right), 7.10\left(\mathrm{~d},{ }^{3} J=19.2 \mathrm{~Hz}, 1 \mathrm{H}, 2-\mathrm{H}\right) \mathrm{ppm}$. ${ }^{13} \mathrm{C}$ NMR (75.6 MHz, $\mathrm{CDCl}_{3}$ additional APT): $\delta=-5.8[+, 2 \mathrm{C}$, $\left.\mathrm{Si}\left(\mathrm{CH}_{3}\right)_{2}\right], 16.9$ [-, $\left.\mathrm{C}_{\text {quat }}, C\left(\mathrm{CH}_{3}\right)_{3}\right], 22.4\left(-, \mathrm{CH}_{2}\right), 24.9\left(-, \mathrm{CH}_{2}\right)$, $26.8\left[+, 3 \mathrm{C}, \mathrm{C}\left(\mathrm{CH}_{3}\right)_{3}\right] 27.4\left(-, \mathrm{CH}_{2}\right), 38.1\left(-, \mathrm{CH}_{2}\right), 126.0\left(-, \mathrm{C}_{\text {quat }}\right)$, 127.8 (+, CH, C-1), $133.2\left(-, \mathrm{C}_{\text {quat }}\right), 145.2(+, \mathrm{CH}, \mathrm{C}-2) \mathrm{ppm} . \mathrm{MS}$ $(70 \mathrm{eV}): m / z(\%)=302 / 300(18 / 18)\left[\mathrm{M}^{+}\right], 246 / 244(38 / 38), 245 / 243$ (100/97), 223 (21), 221 (10), 201 (4), 189 (7), 175 (10), 167 (13), 165 (17), 163 (30), 149 (12), 147 (27), 139/137 (74/76), 135 (8), 123 (13), 121 (17), 105 (54), 95 (13), 93 (19), 91 (24), 83 (14), 79 (27), 73 (45), 67 (12), 59 (49), 57 (46). HRMS: calcd. for $\mathrm{C}_{14} \mathrm{H}_{25} \mathrm{BrSi}$ 300.0909 (correct HRMS).

5-(2-Bromocyclohex-1-en-1-yl)-2,3-dihydro-1,4-dioxine (15): According to GP 2, the bromoenol triflate $\mathbf{1 3}(309 \mathrm{mg}, 1.00 \mathrm{mmol})$ in DMF $(10 \mathrm{~mL})$ and the alkenylstannane $25(450 \mathrm{mg}, 1.20 \mathrm{mmol})$, after treatment with tetrakis(triphenylphosphane)palladium (116 mg, $100 \mu \mathrm{mol}), \mathrm{LiCl}(127 \mathrm{mg}, 3.00 \mathrm{mmol})$ at $90^{\circ} \mathrm{C}$ for $14 \mathrm{~h}$ and workup with diethyl ether $(50 \mathrm{~mL})$, water $(2 \times 20 \mathrm{~mL})$, extraction with diethyl ether $(2 \times 25 \mathrm{~mL})$ and FC ( $45 \mathrm{~g}$ of silica gel, 10:1 $\mathrm{v} / \mathrm{v}$ pentane/diethyl ether elution), gave compound 15 (201 mg, $82 \%$ ) as a clear, colorless oil. $R_{\mathrm{f}}=0.5$. IR (film): $\tilde{v}=2931 \mathrm{~cm}^{-1}$, 2870, 1666, 1642, 1554, 1435, 1368, 1333, 1308, 1283, 1257, 1229, $1155,1114,1091,1027,982,944,920,878,829,795,750 .{ }^{1} \mathrm{H}$ NMR $\left(300 \mathrm{MHz}, \mathrm{CDCl}_{3}\right): \delta=1.56-1.85\left[\mathrm{mc}, 4 \mathrm{H}, 4^{\prime}\left(5^{\prime}\right)-\mathrm{H}\right], 2.23\left(\mathrm{~m}_{\mathrm{c}}, 2\right.$ $\left.\mathrm{H}, 6^{\prime}-\mathrm{H}\right), 2.54$ (m, $\left.2 \mathrm{H}, 3^{\prime}-\mathrm{H}\right), 4.09\left[\mathrm{~m}_{\mathrm{c}}, 4 \mathrm{H}, 2(3)-\mathrm{H}\right], 6.16$ (s, $1 \mathrm{H}$, 6-H) ppm. ${ }^{13} \mathrm{C}$ NMR $\left(75.6 \mathrm{MHz}, \mathrm{CDCl}_{3}\right.$, add. APT): $\delta=22.1(-$, $\left.\mathrm{CH}_{2}\right), 24.42\left(-, \mathrm{CH}_{2}\right), 30.7\left(-, \mathrm{CH}_{2}\right), 37.1\left(-, \mathrm{CH}_{2}\right), 64.2\left(-, \mathrm{CH}_{2}\right.$, $\left.-\mathrm{OCH}_{2}-\right), 64.4\left(-, \mathrm{CH}_{2},-\mathrm{OCH}_{2}-\right), 122.3\left(-, \mathrm{C}_{\text {quat }}\right), 126.6(+, \mathrm{CH}$, C-6), 139.5 (-, $\left.\mathrm{C}_{\text {quat }}\right), 136.7\left(-, \mathrm{C}_{\text {quat }}\right) \mathrm{ppm}$. MS $(70 \mathrm{eV}): \mathrm{m} / \mathrm{z}(\%)=$ 246/244 (95/95) [M+], 235 (8), 189/187 (99/100), 179/177 (8/8), 165 (40), 137 (10), 121 (10), 108 (10), 105 (7), 93 (14), 91 (26), 81 (12), 79 (85), 77 (55), 65 (20), 57 (7). HRMS: calcd. for $\mathrm{C}_{10} \mathrm{H}_{13} \mathrm{BrO}_{2}$ 244.0100 (correct HRMS).

1-Benzyl-4-(2-bromocyclohex-1-enyl)-1,2,3,6-tetrahydropyridine (16): According to GP 2, the bromoenol triflate 13 (309 mg, $1.00 \mathrm{mmol})$ in NMP $(5.00 \mathrm{~mL})$ and the alkenylstannane $\mathbf{4 b}$ $(505 \mathrm{mg}, 1.09 \mathrm{mmol})$, after treatment with $\left[\mathrm{Pd}_{2}(\mathrm{dba})_{3}\right](46.0 \mathrm{mg}$, $50.2 \mu \mathrm{mol}), \mathrm{LiCl}(127 \mathrm{mg}, 3.00 \mathrm{mmol})$ at $65^{\circ} \mathrm{C}$ for $5 \mathrm{~h}$, workup with diethyl ether $(50 \mathrm{~mL})$, water $(2 \times 20 \mathrm{~mL})$, extraction with diethyl ether $(2 \times 20 \mathrm{~mL})$ and FC (30 g on silica gel, $1: 1 \mathrm{v} / \mathrm{v}$ pentane/diethyl ether elution), gave compound $\mathbf{1 6}(279 \mathrm{mg}, 84 \%)$ as a clear, yellow oil. $R_{\mathrm{f}}=0.4$. IR (film): $\tilde{v}=3027 \mathrm{~cm}^{-1}, 2927,2858,2798,1622$, $1595,1494,1453,1367,1327,1158,1126,1074,1028,977,877$,
776, 729. ${ }^{1} \mathrm{H}$ NMR $\left(300 \mathrm{MHz}, \mathrm{CDCl}_{3}\right): \delta=1.62-1.79\left[\mathrm{~m}_{\mathrm{c}}, 4 \mathrm{H}\right.$, $\left.4^{\prime}\left(5^{\prime}\right)-\mathrm{H}\right], 2.10-2.30(\mathrm{~m}, 3 \mathrm{H}), 2.48-2.59(\mathrm{~m}, 3 \mathrm{H}), 2.64\left(\mathrm{t},{ }^{3} \mathrm{~J}=\right.$ $5.5 \mathrm{~Hz}, 2 \mathrm{H}), 3.08\left(\mathrm{~m}_{\mathrm{c}}, 2 \mathrm{H}\right), 3.64\left(\mathrm{~s}, 2 \mathrm{H}, \mathrm{PhCH}_{2}\right), 5.46\left(\mathrm{~m}_{\mathrm{c}}, 1 \mathrm{H}\right.$, 3-H), 7.24-7.42 (m, $5 \mathrm{H}, \mathrm{Ph}-H$ ) ppm. ${ }^{13} \mathrm{C} \mathrm{NMR}\left(75.6 \mathrm{MHz}, \mathrm{CDCl}_{3}\right.$ add. APT): $\delta=22.9\left(-, \mathrm{CH}_{2}\right), 24.6\left(-, \mathrm{CH}_{2}\right), 27.9\left(-, \mathrm{CH}_{2}\right), 31.3$ $\left(-, \mathrm{CH}_{2}\right), 36.7\left(-, \mathrm{CH}_{2}\right), 49.8\left(-, \mathrm{CH}_{2}\right), 52.5\left(-, \mathrm{CH}_{2}\right), 62.7\left(-, \mathrm{CH}_{2}\right.$, $\mathrm{PhCH}_{2}$ ), 118.4 (-, $\left.\mathrm{C}_{\text {quat }}\right), 122.7$ (+, $\left.\mathrm{CH}, \mathrm{C}-3\right), 127.3$ (+, $\left.\mathrm{CH}, \mathrm{Ar}\right)$, 128.5 (+, 2 C, CH, Ar), 129.5 (+, $3 \mathrm{C}), 138.5$ (-, $\left.\mathrm{C}_{\text {quat }}\right), 138.9$ (-, $\left.\mathrm{C}_{\text {quat }}\right)$ ppm. MS $(70 \mathrm{eV}): m / z(\%)=333 / 331(33 / 33)\left[\mathrm{M}^{+}\right], 252(88)$ $\left[\mathrm{M}^{+}-\mathrm{Br}\right], 234$ (13), 233 (10), 172 (33), 158 (17), 133 (20), 117 (11), 105 (17), 91 (100) [ $\left.\mathrm{Bn}^{+}\right], 77$ (18), 65 (21). HRMS: calcd. for $\mathrm{C}_{18} \mathrm{H}_{22} \mathrm{BrN} 331.0937$ (correct HRMS).

trans-2-(2' -Bromocyclohex-1' -enyl)-3,4,4a,5,6,7,8,8a-octahydronaphthalene (17): According to GP 2, the bromoenol triflate 13 $(403 \mathrm{mg}, 1.30 \mathrm{mmol})$ with the bicycloalkenylstannane $8(424 \mathrm{mg}$, $1.00 \mathrm{mmol})$ in DMF $(8 \mathrm{~mL})$, after treatment with $\left[\mathrm{Pd}_{2}(\mathrm{dba})_{3}\right]$. $\mathrm{CHCl}_{3}(104 \mathrm{mg}, 100 \mu \mathrm{mol}), \mathrm{AsPh}_{3}(24 \mathrm{mg}, 78.0 \mu \mathrm{mol}), \mathrm{LiCl}$ (128 mg, $3 \mathrm{mmol}), \mathrm{CuI}(10 \mathrm{mg}, 52 \mu \mathrm{mol})$, at $65^{\circ} \mathrm{C}$ for $5 \mathrm{~h}$, workup with diethyl ether $(50 \mathrm{~mL})$, water $(2 \times 20 \mathrm{~mL})$, extraction with diethyl ether $(2 \times 35 \mathrm{~mL})$ and FC ( $40 \mathrm{~g}$ of silica gel, light petroleum), gave compound $17(261 \mathrm{mg}, 89 \%)$ as a colorless wax. $R_{\mathrm{f}}=0.7$. IR (film): $\tilde{v}=2918 \mathrm{~cm}^{-1}, 2851,2824,1640,1446,1326,978 .{ }^{1} \mathrm{H} \mathrm{NMR}$ $\left(250 \mathrm{MHz}, \mathrm{CDCl}_{3}\right): \delta=0.80-1.19(\mathrm{~m}, 3 \mathrm{H}), 1.20-1.53(\mathrm{~m}, 3 \mathrm{H})$, $1.55-1.86(\mathrm{~m}, 10 \mathrm{H}), 1.96-2.35\left[\mathrm{~m}_{\text {coalesced }}, 4 \mathrm{H}, 3\left(3^{\prime}\right)-\mathrm{H}\right], 2.47-2.59$ (m, $\left.2 \mathrm{H}, 66^{\prime}-\mathrm{H}\right), 5.20$ (s, $\left.1 \mathrm{H}, 1-\mathrm{H}\right) \mathrm{ppm} .{ }^{13} \mathrm{C} \mathrm{NMR}(62.9 \mathrm{MHz}$, $\mathrm{CDCl}_{3}$, add. DEPT): $\delta=22.6\left(-, \mathrm{CH}_{2}\right), 24.7\left(-, \mathrm{CH}_{2}\right), 26.8(-$, $\left.\mathrm{CH}_{2}\right), 26.9\left(-, \mathrm{CH}_{2}\right), 27.3\left(-, \mathrm{CH}_{2}\right), 30.1\left(-, \mathrm{CH}_{2}\right), 31.9\left(-, \mathrm{CH}_{2}\right)$, $33.0\left(-, \mathrm{CH}_{2}\right), 33.4\left(-, \mathrm{CH}_{2}\right), 36.3\left(-, \mathrm{CH}_{2}\right), 40.4(+, \mathrm{CH}), 42.1(+$, $\mathrm{CH}), 117.7\left(\mathrm{C}_{\text {quat }}, \mathrm{C}-2\right), 129.4(+, \mathrm{C}-\mathrm{H}, \mathrm{C}-1), 139.5\left(\mathrm{C}_{\text {quat }}, \mathrm{C}-1^{\prime}\right)$, $139.6\left(\mathrm{C}_{\text {quat }}, \mathrm{C}-2^{\prime}\right) \mathrm{ppm}$. MS $(70 \mathrm{eV}): \mathrm{m} / \mathrm{z}(\%)=296 / 294(13 / 13)$ $\left[\mathrm{M}^{+}\right], 215$ (100) $\left[\mathrm{M}^{+}-\mathrm{Br}\right], 187$ (7), 133 (10), 95 (6), 91 (6), 67 (4), 41 (3). HRMS: calcd. for $\mathrm{C}_{16} \mathrm{H}_{23} \mathrm{Br} 294.0984$ (correct HRMS).

2-(2' - Bromocyclohex-1' - enyl)-5-tert-butoxy-4a-methyl1,2,3,3,4,4a,5,6,8a-octahydronaphthalene (18): According to GP 2, the bromoenol triflate $13(0.713 \mathrm{~g}, 2.30 \mathrm{mmol})$ with the bicycloalkenylstannane $11(1.02 \mathrm{~g}, 2.00 \mathrm{mmol})$ in NMP $(20 \mathrm{~mL})$, after treatment with $\left[\mathrm{Pd}_{2}(\mathrm{dba})_{3}\right] \cdot \mathrm{CHCl}_{3}(104 \mathrm{mg}, 100 \mu \mathrm{mol}), \mathrm{AsPh}_{3}(24.0 \mathrm{mg}$, $78.0 \mu \mathrm{mol}), \mathrm{LiCl}(384 \mathrm{mg}, 9.05 \mathrm{mmol}), \mathrm{CuI}(10.0 \mathrm{mg}, 52.0 \mu \mathrm{mol})$ at $65^{\circ} \mathrm{C}$ for $5 \mathrm{~h}$ and after workup with diethyl ether $(75 \mathrm{~mL})$, water $(2 \times 25 \mathrm{~mL})$, extraction with diethyl ether $(2 \times 20 \mathrm{~mL})$ and $\mathrm{FC}(90 \mathrm{~g}$ of silica gel, $20: 1 \mathrm{v} / \mathrm{v}$ light petroleum/diethyl ether elution), gave compound $18(716 \mathrm{mg}, 94 \%)$ as a colorless wax. $R_{\mathrm{f}}=0.5$. IR (film): $\tilde{v}=2927 \mathrm{~cm}^{-1}, 2858,1643,1456,1446,1387,1378,1362,1265$, $1248,1137,1124,1075,1050,1019,1000,959,848,740 .{ }^{1} \mathrm{H}$ NMR $\left(250 \mathrm{MHz}, \mathrm{CDCl}_{3}\right): \delta=0.84\left(\mathrm{~s}, 3 \mathrm{H}, \mathrm{CH}_{3}\right), 1.18\left[\mathrm{~s}, 9 \mathrm{H}, \mathrm{C}\left(\mathrm{CH}_{3}\right)_{3}\right]$, 1.21-1.40 (m, $4 \mathrm{H}), 1.43-1.81$ (m, $7 \mathrm{H}), 1.85-2.09$ (m, $2 \mathrm{H}), 2.10$ $2.28(\mathrm{~m}, 4 \mathrm{H}), 2.41-2.55(\mathrm{~m}, 2 \mathrm{H}), 3.08\left(\mathrm{dd},{ }^{3} \mathrm{~J}=6.8,{ }^{3} \mathrm{~J}=11.0 \mathrm{~Hz}\right.$, $1 \mathrm{H}, 5-\mathrm{H}), 5.28-5.31\left(\mathrm{~d},{ }^{3} \mathrm{~J}=1.3 \mathrm{~Hz}, 1 \mathrm{H}, 1-\mathrm{H}\right) \mathrm{ppm} .{ }^{13} \mathrm{C} \mathrm{NMR}$ (62.9 $\mathrm{MHz}, \mathrm{CDCl}_{3}$, add. DEPT): $\delta=10.5\left(+, \mathrm{CH}_{3}\right), 22.5\left(-, \mathrm{CH}_{2}\right)$, $24.4\left(-, \mathrm{CH}_{2}\right), 24.7\left(-, \mathrm{CH}_{2}\right), 24.8\left(-, \mathrm{CH}_{2}\right), 26.7\left(-, \mathrm{CH}_{2}\right), 29.1[+$, $\left.3 \mathrm{C}, \mathrm{C}\left(\mathrm{CH}_{3}\right)_{3}\right], 30.7\left(-, \mathrm{CH}_{2}\right), 32.0\left(-, \mathrm{CH}_{2}\right), 34.1\left(-, \mathrm{CH}_{2}\right), 36.3$ $\left(-, \mathrm{CH}_{2}\right), 37.4\left(\mathrm{C}_{\text {quat }}, \mathrm{C}-4 \mathrm{a}\right), 43.7(+, \mathrm{CH}, \mathrm{C}-8 \mathrm{a}), 72.7\left[\mathrm{C}_{\text {quat }}\right.$, $\left.C\left(\mathrm{CH}_{3}\right)_{3}\right], 77.4(+, \mathrm{CH}, \mathrm{C}-5), 117.8\left(\mathrm{C}_{\text {quat }}, \mathrm{C}-2\right), 127.5(+, \mathrm{C}-1)$, $139.0\left(\mathrm{C}_{\text {quat }}, \mathrm{C}-2^{\prime}\right), 139.4\left(\mathrm{C}_{\text {quat }},{\left.\mathrm{C}-1^{\prime}\right)}^{\prime}\right) \mathrm{ppm} . \mathrm{MS}(70 \mathrm{eV}): \mathrm{m} / \mathrm{z}(\%)$ $=382 / 380(59 / 58)\left[\mathrm{M}^{+}\right], 326 / 324(44 / 52)\left[\mathrm{M}^{+}-\mathrm{C}_{4} \mathrm{H}_{8}\right], 307 / 305(62 /$ 52), 279 (8), 251 (10), 245 (52) [ $\left.\mathrm{M}^{+}-\mathrm{Br}-\mathrm{C}_{4} \mathrm{H}_{8}\right], 243$ (79), 227 (98) $\left[\mathrm{M}^{+}-\mathrm{C}_{4} \mathrm{H}_{8}-\mathrm{H}_{2} \mathrm{O}-\mathrm{Br}\right], 225$ (52), 199 (13), 159 (9), 152 (10), 145 (27), 93 (38), 81/79 (21/20), 57 (100) $\left[\mathrm{C}_{4} \mathrm{H}_{9}{ }^{+}\right], 41$ (25). HRMS: calcd. for $\mathrm{C}_{21} \mathrm{H}_{33} \mathrm{BrO} 380.1717$ (correct HRMS).

tert-Butyl (E)-3-\{2'-[(E)-2' '-(tert-Butyldimethylsilyl)vinyl]cyclohex1'-enyl acrylate (19): According to GP 3, the bromobutadiene 14 $(230 \mathrm{mg}, 0.764 \mathrm{mmol})$ in DMF $(10.0 \mathrm{~mL})$ after treatment with 
$\mathrm{Pd}(\mathrm{OAc})_{2}(17.1 \mathrm{mg}, 0.0760 \mathrm{mmol}), \mathrm{PPh}_{3}(60.1 \mathrm{mg}, 0.229 \mathrm{mmol})$, $\mathrm{NEt}_{3}(232 \mathrm{mg}, 2.29 \mathrm{mmol})$, tert-butyl acrylate $(490 \mathrm{mg}, 3.82 \mathrm{mmol})$ at $90{ }^{\circ} \mathrm{C}$ for $14 \mathrm{~h}$, workup with diethyl ether $(50 \mathrm{~mL})$, water $(20 \mathrm{~mL})$, extraction with diethyl ether $(25 \mathrm{~mL})$ and FC $(30 \mathrm{~g}$ on silica gel, 20:1 v/v pentane/diethyl ether elution), gave the 1,3,5hexatriene 19 as a colorless solid (208 mg, 78\%), m.p. $155-157{ }^{\circ} \mathrm{C}$. Good-quality crystals for X-ray diffraction were grown from pentane/diethyl ether $(20: 1)$ at $23^{\circ} \mathrm{C}$ by slow evaporation of the solvents. $R_{\mathrm{f}}=0.3$. IR (film): $\tilde{v}=2951 \mathrm{~cm}^{-1}, 2930,2883,2857,1708$, $1621,1470,1462,1391,1366,1340,1305,1278,1248,1202,1147$, 1070, 1038, 1007, 981, 938, 828, 813, 792, 751, 728. ${ }^{1} \mathrm{H}$ NMR $\left(300 \mathrm{MHz}, \mathrm{CDCl}_{3}\right): \delta=0.09\left[\mathrm{~s}, 6 \mathrm{H}, \mathrm{Si}\left(\mathrm{CH}_{3}\right)_{2}\right], 0.89[\mathrm{~s}, 9 \mathrm{H}$, $\left.\mathrm{SiC}\left(\mathrm{CH}_{3}\right)_{3}\right], 1.15$ [s, $\left.9 \mathrm{H}, \mathrm{CO}_{2} \mathrm{C}\left(\mathrm{CH}_{3}\right)_{3}\right], 1.59-1.76\left[\mathrm{~m}, 4 \mathrm{H}, 4^{\prime}\left(5^{\prime}\right)-\right.$ $\mathrm{H}], 2.28\left(\mathrm{~m}_{\mathrm{c}}, 2 \mathrm{H}, 3^{\prime}-\mathrm{H}\right), 2.36\left(\mathrm{~m}, 2 \mathrm{H}, 6^{\prime}-\mathrm{H}\right), 5.81\left(\mathrm{~d},{ }^{3} J=15.4 \mathrm{~Hz}\right.$, $1 \mathrm{H}, 3-\mathrm{H}), 6.05\left(\mathrm{~d},{ }^{3} J=18.7 \mathrm{~Hz}, 1 \mathrm{H}, 1^{\prime \prime}-\mathrm{H}\right), 7.33\left(\mathrm{~d},{ }^{3} J=18.9 \mathrm{~Hz}\right.$, $\left.1 \mathrm{H}, 22^{\prime \prime}-\mathrm{H}\right), 8.06\left(\mathrm{~d},{ }^{3} \mathrm{~J}=15.7 \mathrm{~Hz}, 1 \mathrm{H}, 2-\mathrm{H}\right) \mathrm{ppm} .{ }^{13} \mathrm{C} \mathrm{NMR}$ (75.6 MHz, $\mathrm{CDCl}_{3}$, add. APT): $\delta=-5.8\left[+, 2 \mathrm{C}, \mathrm{CH}_{3}, \mathrm{Si}\left(\mathrm{CH}_{3}\right)_{2}\right]$, $16.9\left[-, \mathrm{C}_{\text {quat }}, C\left(\mathrm{CH}_{3}\right)_{3}\right], 22.4\left(-, \mathrm{CH}_{2}\right), 22.5\left(-, \mathrm{CH}_{2}\right), 26.5\left(-, \mathrm{CH}_{2}\right)$, $26.8\left[+, 3 \mathrm{C}, \mathrm{CH}_{3}, \mathrm{C}\left(\mathrm{CH}_{3}\right)_{3}\right], 26.9\left(-, \mathrm{CH}_{2}\right), 28.4[+, 3 \mathrm{C}$, $\left.\mathrm{CO}_{2} \mathrm{C}\left(\mathrm{CH}_{3}\right)_{3}\right], 80.2\left[-, \mathrm{C}_{\text {quat }}, \mathrm{CH}_{3}, \mathrm{C}\left(\mathrm{CH}_{3}\right)_{3}\right], 118.6\left(-, \mathrm{C}_{\text {quat }}\right), 129.0$ $\left(+, \mathrm{CH}, \mathrm{C}-1^{\prime \prime}\right), 131.6\left(-, \mathrm{C}_{\text {quat }}\right), 140.5\left(+, \mathrm{CH}, \mathrm{C}-2^{\prime \prime}\right), 141.2(+$, $\mathrm{CH}, \mathrm{C}-3), 141.3(+, \mathrm{CH}, \mathrm{C}-2), 175.6\left(-, \mathrm{C}_{\text {quat }}, \mathrm{C}=\mathrm{O}\right) \mathrm{ppm} . \mathrm{MS}$ $(70 \mathrm{eV}): m / z(\%)=348(7)\left[\mathrm{M}^{+}\right], 292(9)\left[\mathrm{M}^{+}-\mathrm{C}_{4} \mathrm{H}_{8}\right], 247(11), 235$ (100), 207 (9), 191 (7), 189 (20), 161 (14), 159 (6), 132 (6), 131 (18), 116 (6), 115 (32) 103 (7), 91 (10), 75 (68), 57 (21) $\left[\mathrm{C}_{4} \mathrm{H}_{9}^{+}\right]$. HRMS: calcd. for $\mathrm{C}_{21} \mathrm{H}_{36} \mathrm{O}_{2} \mathrm{Si} 348.2477$ (correct HRMS).

tert-Butyl (E)-3-\{2'-[(E)-2' '-(Trimethylsilyl)vinyl]cyclohex-1' enyl acrylate (19-SiMe $\mathbf{S}_{3}$ ): According to GP 3, the bromobutadiene 14-SiMe 3 (200 mg, $0.775 \mathrm{mmol})$ in DMF $(5.00 \mathrm{~mL})$, after treatment with $\mathrm{Pd}(\mathrm{OAc})_{2}(17.4 \mathrm{mg}, 0.0780 \mathrm{mmol}), \mathrm{PPh}_{3}(61.0 \mathrm{mg}$, $0.233 \mathrm{mmol}), \mathrm{NEt}_{3}(235 \mathrm{mg}, 2.33 \mathrm{mmol})$, tert-butyl acrylate (497 mg, $3.88 \mathrm{mmol}$ ) at $90{ }^{\circ} \mathrm{C}$ for $14 \mathrm{~h}$, workup with diethyl ether $(50 \mathrm{~mL})$, water $(20 \mathrm{~mL})$ extraction with diethyl ether $(25 \mathrm{~mL})$ and FC (24 g of silica gel, 20:1 v/v pentane/diethyl ether elution), gave 19- $\mathrm{SiMe}_{3}(211 \mathrm{mg}, 89 \%)$ as a colorless wax. The analytical data obtained on this material are identical with those reported previously. ${ }^{[7 b]}$

tert-Butyl (E)-3-[2'-(5', $6^{\prime \prime}$-Dihydro-1' ${ }^{\prime \prime}, 4^{\prime \prime}$-dioxin-2' '-yl)cyclohex$1^{\prime}$-enyl]acrylate (20): According to GP 3, the bromobutadiene 15 (230 mg, $0.764 \mathrm{mmol})$ in DMF/water $(10: 1)(1.10 \mathrm{~mL})$, after treatment with the palladacycle $(7.6 \mathrm{mg}, 8.2 \mu \mathrm{mol})$, NaOAc $(40.2 \mathrm{mg}$, $0.490 \mathrm{mmol}), n \mathrm{Bu}_{4} \mathrm{NBr}(52.5 \mathrm{mg}, 0.163 \mathrm{mmol})$, tert-butyl acrylate (105 mg, $0.816 \mathrm{mmol}$ ) at $105^{\circ} \mathrm{C}$ for $12 \mathrm{~h}$, workup with diethyl ether $(30 \mathrm{~mL})$, water $(2 \times 10 \mathrm{~mL})$ extraction with diethyl ether $(10 \mathrm{~mL})$ and FC (18 $\mathrm{g}$ of silica gel, 5:1 v/v pentane/diethyl ether elution), gave compound $20(31.1 \mathrm{mg}, 65 \%)$ as a colorless wax. $R_{\mathrm{f}}=0.4$. IR (film): $\tilde{v}=2974 \mathrm{~cm}^{-1}, 2930,2871,1703,1640,1608,1458,1391$, 1366, 1311, 1278, 1258, 1150, 1091, 1028, 985, 922, 882, 855, 796. ${ }^{1} \mathrm{H}$ NMR $\left(300 \mathrm{MHz}, \mathrm{C}_{6} \mathrm{D}_{6}\right): \delta=1.49\left[\mathrm{~s}, 9 \mathrm{H}, \mathrm{CO}_{2} \mathrm{C}\left(\mathrm{CH}_{3}\right)_{3}\right], 1.57-$ $1.71\left[\mathrm{~m}, 4 \mathrm{H}, 4^{\prime}\left(5^{\prime}\right)-\mathrm{H}\right], 2.16-2.24$ (m, $\left.2 \mathrm{H}, 6^{\prime}-\mathrm{H}\right), 2.26-2.35$ (m, 2 $\left.\mathrm{H}, 3^{\prime}-\mathrm{H}\right), 4.11\left[\mathrm{~m}_{\mathrm{c}}, 4 \mathrm{H}, 5^{\prime \prime}\left(6^{\prime \prime}\right)-\mathrm{H}\right], 5.74\left(\mathrm{~d},{ }^{3} \mathrm{~J}=15.9 \mathrm{~Hz}, 1 \mathrm{H}, 3-\right.$ $\mathrm{H}), 5.96\left(\mathrm{~m}_{\mathrm{c}}, 1 \mathrm{H}, 3^{\prime \prime}{ }^{\prime} \mathrm{H}\right), 7.88\left(\mathrm{~d},{ }^{3} J=15.9 \mathrm{~Hz}, 1 \mathrm{H}, 2-\mathrm{H}\right) \mathrm{ppm}$. ${ }^{13} \mathrm{C} \mathrm{NMR}\left(75.6 \mathrm{MHz}, \mathrm{CDCl}_{3}\right.$, add. APT): $\delta=22.09\left(-, \mathrm{CH}_{2}\right), 22.14$ $\left(-, \mathrm{CH}_{2}\right), 25.87\left(-, \mathrm{CH}_{2}\right), 28.20\left[+, 3 \mathrm{C}, \mathrm{CO}_{2} \mathrm{C}\left(\mathrm{CH}_{3}\right)_{3}\right], 28.88(-$, $\left.\mathrm{CH}_{2}\right), 64.20\left(-, \mathrm{CH}_{2},-\mathrm{OCH}_{2}^{-}\right), 64.34\left(-, \mathrm{CH}_{2},-\mathrm{OCH}_{2}-\right), 79.74$ [-, $\left.\mathrm{C}_{\text {quat }}, \mathrm{CO}_{2} \mathrm{C}\left(\mathrm{CH}_{3}\right)_{3}\right], 117.16(+, \mathrm{CH}, \mathrm{C}-3), 128.06\left(+, \mathrm{CH}, \mathrm{C}-3^{\prime \prime}\right)$, $131.18\left(-, \mathrm{C}_{\text {quat }}\right), 135.71\left(-, \mathrm{C}_{\text {quat }}\right), 138.34\left(-, \mathrm{C}_{\text {quat }}\right), 143.42(+, \mathrm{CH}$, $\mathrm{C}-2), 167.25\left(-, \mathrm{C}_{\text {quat }}, \mathrm{C}=\mathrm{O}\right) \mathrm{ppm}$. MS $(70 \mathrm{eV}): \mathrm{m} / \mathrm{z}(\%)=292(7)$ $\left[\mathrm{M}^{+}\right], 236(31)\left[\mathrm{M}^{+}-\mathrm{C}_{4} \mathrm{H}_{8}\right], 235(34)\left[\mathrm{M}^{+}-\mathrm{C}_{4} \mathrm{H}_{9}\right], 219$ (12), 217 (7), 191 (100), 162 (6), 149 (67), 134 (6), 105 (7), 91 (15), 77 (8), 57 (26) $\left[\mathrm{C}_{4} \mathrm{H}_{9}{ }^{+}\right]$. HRMS: calcd. for $\mathrm{C}_{17} \mathrm{H}_{24} \mathrm{O}_{4} 292.1676$ (correct HRMS).
tert-Butyl (E)-3-[2'-(1' ${ }^{\prime \prime}-$ Benzyl-1" ${ }^{\prime \prime}, 2^{\prime \prime}, 3^{\prime \prime}, 6^{\prime \prime}$-tetrahydropyridin-4" yl)cyclohex-1' -enyl|acrylate (21): According to GP 3, the bromobutadiene 16 (110 mg, $0.331 \mathrm{mmol})$ in DMF/water (10:1) $(2.2 \mathrm{~mL})$ was treated with the palladacycle $(16.0 \mathrm{mg}, 16.6 \mu \mathrm{mol}), \mathrm{NaOAc}$ (82.0 mg, $0.993 \mathrm{mmol}), n \mathrm{Bu}_{4} \mathrm{NBr}(70.0 \mathrm{mg}, 0.331 \mathrm{mmol})$, tert-butyl acrylate $(1.00 \mathrm{~mL})$ at $105^{\circ} \mathrm{C}$ for $4 \mathrm{~h}$. For complete consumption of the bromobutadiene $\mathbf{1 6}$, a second portion of the palladacycle $(16.0 \mathrm{mg}, 16.6 \mu \mathrm{mol})$ was added and the mixture again heated at $105^{\circ} \mathrm{C}$ for $4 \mathrm{~h}$. Workup with diethyl ether $(45 \mathrm{~mL})$, water $(15 \mathrm{~mL})$, extraction with diethyl ether $(15 \mathrm{~mL})$ and FC (22 $\mathrm{g}$ of silica gel, $10: 1 \mathrm{v} / \mathrm{v}$ diethyl ether/methanol elution) gave compound 21 $(89.2 \mathrm{mg}, 71 \%)$ as a colorless wax. $R_{\mathrm{f}}=0.5$. IR (film): $\tilde{\mathrm{v}}=$ $3024 \mathrm{~cm}^{-1}, 2974,2930,2862,1761,1703,1614,1476,1452,1390$, 1366, 1310, 1275, 1257, 1149, 1049, 985, 884, 855, 733. ${ }^{1} \mathrm{H}$ NMR $\left(300 \mathrm{MHz}, \mathrm{CDCl}_{3}\right): \delta=1.25-1.38\left[\mathrm{~m}, 4 \mathrm{H}, 4^{\prime}\left(5^{\prime}\right)-\mathrm{H}\right], 1.47[\mathrm{~s}, 9 \mathrm{H}$, $\left.\mathrm{CO}_{2} \mathrm{C}\left(\mathrm{CH}_{3}\right)_{3}\right], 1.91-2.01(\mathrm{~m}, 4 \mathrm{H}), 2.02-2.11(\mathrm{~m}, 2 \mathrm{H}), 2.44\left(\mathrm{t},{ }^{3} \mathrm{~J}=\right.$ $5.5 \mathrm{~Hz}, 2 \mathrm{H}), 2.87\left(\mathrm{~m}_{\mathrm{c}}, 2 \mathrm{H}\right), 3.38\left(\mathrm{~s}, 2 \mathrm{H}, \mathrm{PhCH}_{2}\right), 5.33\left(\mathrm{~m}_{\mathrm{c}}, 1 \mathrm{H}\right.$, $\left.3^{\prime \prime}-\mathrm{H}\right), 6.01\left(\mathrm{~d},{ }^{3} J=15.7 \mathrm{~Hz}, 1 \mathrm{H}, 3-\mathrm{H}\right), 7.09-7.22(\mathrm{~m}, 3 \mathrm{H}, \mathrm{Ph}-H)$, 7.35-7.45 (m, $2 \mathrm{H}, \mathrm{Ph}-H), 8.19\left(\mathrm{~d},{ }^{3} J=15.8 \mathrm{~Hz}, 1 \mathrm{H}, 2-\mathrm{H}\right) \mathrm{ppm}$. ${ }^{13} \mathrm{C} \mathrm{NMR}\left(75.6 \mathrm{MHz}, \mathrm{CDCl}_{3}\right.$, add. APT): $\delta=22.5\left(-, \mathrm{CH}_{2}\right), 22.6$

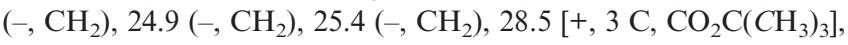
$30.5\left(-, \mathrm{CH}_{2}\right), 49.7\left(-, \mathrm{CH}_{2}\right), 52.9\left(-, \mathrm{CH}_{2}\right), 62.9\left(-, \mathrm{CH}_{2}, \mathrm{PhCH}_{2}\right)$, $79.9\left[\mathrm{C}_{\text {quat }}, \mathrm{CO}_{2} \mathrm{C}\left(\mathrm{CH}_{3}\right)_{3}\right], 116.7\left(+, \mathrm{CH}, \mathrm{C}-3^{\prime \prime}\right), 124.7(+, \mathrm{CH})$, $127.3(+, \mathrm{CH}, \mathrm{Ph}), 128.0$ (-, $\left.\mathrm{C}_{\text {quat }}\right), 128.5(+, 2 \mathrm{C}, \mathrm{CH}, \mathrm{Ph}), 129.4$ $(+, 2 \mathrm{C}, \mathrm{CH}, \mathrm{Ph}), 136.9\left(-, \mathrm{C}_{\text {quat }}\right), 138.5\left(-, \mathrm{C}_{\text {quat }}, \mathrm{Ph}\right), 144.2(+$, $\mathrm{CH}, \mathrm{C}-2), 149.2\left(-, \mathrm{C}_{\text {quat }}\right), 167.7\left(-, \mathrm{C}_{\text {quat }}, \mathrm{C}=\mathrm{O}\right) \mathrm{ppm}$. MS $(70 \mathrm{eV})$ : $m / z(\%)=379(6)\left[\mathrm{M}^{+}\right], 333(7), 323(7)\left[\mathrm{M}^{+}-\mathrm{C}_{4} \mathrm{H}_{8}\right], 322(22)$ $\left[\mathrm{M}^{+}-\mathrm{C}_{4} \mathrm{H}_{9}\right], 278$ (6), 252 (33), 220 (5), 205 (17), 172 (7), 149 (7), 135 (9), 122 (24), 105 (37), 91 (100) [ $\left.\mathrm{Bn}^{+}\right], 77$ (32), 65 (11), 57 (22) $\left[\mathrm{C}_{4} \mathrm{H}_{9}{ }^{+}\right]$. HRMS: calcd. for $\mathrm{C}_{25} \mathrm{H}_{33} \mathrm{NO}_{2} 379.2513$ (correct HRMS).

tert-Butyl (E)-3-[2'-(trans-3' ${ }^{\prime \prime}, 4^{\prime \prime}, 4 \mathrm{a}^{\prime \prime}, 5^{\prime \prime}, 6^{\prime \prime}, 7^{\prime \prime}, 8^{\prime \prime}, 8 \mathrm{a}^{\prime \prime}$-Octahydronaphth-2' '-enyl)cyclohex-1' -enyl|acrylate (22- $t \mathrm{Bu})$ : According to GP 3, the bromobutadiene 17 (340 $\mathrm{mg}, 1.15 \mathrm{mmol})$ in DMF/ $\mathrm{MeCN} / \mathrm{H}_{2} \mathrm{O}(10: 5: 1)(6 \mathrm{~mL})$ was treated with the palladacycle (41.0 mg, $46.0 \mu \mathrm{mol}), n \mathrm{Bu}_{4} \mathrm{NOAc}(722 \mathrm{mg}, 2.39 \mathrm{mmol})$, tert-butyl acrylate $(2.5 \mathrm{~mL})$ at $105^{\circ} \mathrm{C}$ for $4 \mathrm{~h}$. For complete consumption of the bromobutadiene $\mathbf{1 7}$, a second portion of the palladacycle $(41.0 \mathrm{mg}, 46.0 \mu \mathrm{mol})$ was added and the mixture again heated at $105^{\circ} \mathrm{C}$ for $4 \mathrm{~h}$. Workup with diethyl ether $(50 \mathrm{~mL})$, water $(2 \times 30 \mathrm{~mL})$, extraction with diethyl ether $(50 \mathrm{~mL})$ and FC $(31 \mathrm{~g}$ of silica gel, 20:1 v/v light petroleum/diethyl ether elution) gave compound $22-t \mathrm{Bu}(228 \mathrm{mg}, 58 \%)$ as a colorless wax. $R_{\mathrm{f}}=0.2$. IR (film): $\tilde{v}=2976 \mathrm{~cm}^{-1}, 2922,2852,1704,1613,1449,1391,1367$, 1311, 1296, 1273, 1255, 1207, 1149, 1068, 1036, 986, 911, 885, 854, $825,734,648 .{ }^{1} \mathrm{H}$ NMR $\left(250 \mathrm{MHz}, \mathrm{C}_{6} \mathrm{D}_{6}\right): \delta=0.83-1.13(\mathrm{~m}, 4 \mathrm{H})$, $1.14-1.23(\mathrm{~m}, 7 \mathrm{H}), 1.28\left[\mathrm{~s}, 9 \mathrm{H}, \mathrm{C}\left(\mathrm{CH}_{3}\right)_{3}\right], 1.29-1.76(\mathrm{~m}, 5 \mathrm{H})$, $1.95-2.13(\mathrm{~m}, 6 \mathrm{H}), 5.14\left(\mathrm{~s}, 1 \mathrm{H}, 1^{\prime \prime}-\mathrm{H}\right), 5.98\left(\mathrm{~d},{ }^{3} \mathrm{~J}=15.8 \mathrm{~Hz}, 1 \mathrm{H}\right.$, 2-H), $8.12\left(\mathrm{~d},{ }^{3} J=15.8 \mathrm{~Hz}, 1 \mathrm{H}, 3-\mathrm{H}\right) \mathrm{ppm} .{ }^{13} \mathrm{C} \mathrm{NMR}(62.9 \mathrm{MHz}$, $\mathrm{C}_{6} \mathrm{D}_{6}$, add. DEPT): $\delta=22.6\left(-, \mathrm{CH}_{2}\right), 22.7\left(-, \mathrm{CH}_{2}\right), 25.3\left(-, \mathrm{CH}_{2}\right)$, $27.0\left(-, \mathrm{CH}_{2}\right), 27.2\left(-, \mathrm{CH}_{2}\right), 28.3\left[+, 3 \mathrm{C}, \mathrm{C}\left(\mathrm{CH}_{3}\right)_{3}\right], 29.2\left(-, \mathrm{CH}_{2}\right)$, $30.4\left(-, \mathrm{CH}_{2}\right), 30.8\left(-, \mathrm{CH}_{2}\right), 33.5\left(-, \mathrm{CH}_{2}\right), 33.6\left(-, \mathrm{CH}_{2}\right), 40.8(+$, $\mathrm{CH}), 42.5(+, \mathrm{CH}), 79.2\left[\mathrm{C}_{\text {quat }}, \mathrm{CO}_{2} \mathrm{C}\left(\mathrm{CH}_{3}\right)_{3}\right], 117.0(+, \mathrm{C}-2), 128.0$ $\left(\mathrm{C}_{\text {quat }}\right), 132.0\left(+, \mathrm{C}-1^{\prime \prime}\right), 138.2\left(\mathrm{C}_{\text {quat }}\right), 144.4(+, \mathrm{C}-3), 149.7\left(\mathrm{C}_{\text {quat }}\right)$, $167.8\left(\mathrm{C}_{\text {quat }}, \mathrm{C}=\mathrm{O}\right) \mathrm{ppm}$. MS $(70 \mathrm{eV}): m / z(\%)=342(1)\left[\mathrm{M}^{+}\right], 286$ (21) $\left[\mathrm{M}^{+}-\mathrm{C}_{4} \mathrm{H}_{8}\right], 241$ (12), 226 (10), 218 (2), 192 (11), 190 (10), 162 (4), 146 (14), 145 (100), 105 (14), 91 (22), 84 (48), 69 (17), 57 (66) $\left[\mathrm{C}_{4} \mathrm{H}_{9}^{+}\right], 56(65)\left[\mathrm{C}_{4} \mathrm{H}_{8}^{+}\right], 41$ (56). HRMS: calcd. for $\mathrm{C}_{23} \mathrm{H}_{34} \mathrm{O}_{2}$ 342.2561 (correct HRMS).

Methyl (E)-3-[2'-trans- $\left(3^{\prime \prime}, 4^{\prime \prime}, 4 a^{\prime \prime}, 5^{\prime \prime}, 6^{\prime \prime}, 7^{\prime \prime}, 8^{\prime \prime}, 8 a^{\prime \prime}\right.$-Octahydronaphth-2' '-enyl)cyclohex-1'-enyl|acrylate (22-Me): According to GP 3, bromobutadiene 17 (205 mg, $0.695 \mathrm{mmol})$ in $\mathrm{DMF} / \mathrm{MeCN} / \mathrm{H}_{2} \mathrm{O}$ $(10: 5: 1)(6 \mathrm{~mL})$ was treated with the palladacycle $(49.0 \mathrm{mg}$, $55.0 \mu \mathrm{mol}), n \mathrm{Bu}_{4} \mathrm{NOAc}(418 \mathrm{mg}, 1.39 \mathrm{mmol})$ and methyl acrylate 
(298 mg, $3.48 \mathrm{mmol}$ ) at $105^{\circ} \mathrm{C}$ for $4 \mathrm{~h}$. For complete consumption of 17 , a second portion of the palladacycle $(49.0 \mathrm{mg}, 55.0 \mu \mathrm{mol})$ was added, and the mixture again heated at $105^{\circ} \mathrm{C}$ for $4 \mathrm{~h}$. Workup with diethyl ether $(50 \mathrm{~mL})$, water $(2 \times 30 \mathrm{~mL})$, extraction with diethyl ether $(50 \mathrm{~mL})$ and FC (27 g of silica gel, 20:1 v/v light petroleum/diethyl ether elution) gave compound 22-Me (176 mg, 84\%) as a colorless wax. $R_{\mathrm{f}}=0.3$. IR (film): $\tilde{v}=2921 \mathrm{~cm}^{-1}, 2851,1721$, 1615, 1433, 1296, 1273, 1164, 1134, 1068, 1037, 1018, 923, 880, $852,752,665 .{ }^{1} \mathrm{H}$ NMR $\left(250 \mathrm{MHz}, \mathrm{CDCl}_{3}\right): \delta=0.90-1.11(\mathrm{~m}$, $3 \mathrm{H}), 1.13-1.47(\mathrm{~m}, 3 \mathrm{H}), 1.52-1.80(\mathrm{~m}, 10 \mathrm{H}), 1.94-2.28(\mathrm{~m}, 6 \mathrm{H})$, $3.72\left(\mathrm{~s}, 3 \mathrm{H}, \mathrm{CH}_{3}\right), 5.14\left(\mathrm{~s}, 1 \mathrm{H}, 1^{\prime \prime}-\mathrm{H}\right), 5.71\left(\mathrm{~d},{ }^{3} \mathrm{~J}=15.1 \mathrm{~Hz}, 1 \mathrm{H}\right.$, 2-H), $7.78\left(\mathrm{~d},{ }^{3} \mathrm{~J}=15.1 \mathrm{~Hz}, 1 \mathrm{H}, 3-\mathrm{H}\right) \mathrm{ppm} .{ }^{13} \mathrm{C} \mathrm{NMR}(62.9 \mathrm{MHz}$, $\mathrm{CDCl}_{3}$, add. DEPT): $\delta=22.2\left(-, \mathrm{CH}_{2}\right), 22.4\left(-, \mathrm{CH}_{2}\right), 25.1(-$, $\left.\mathrm{CH}_{2}\right), 26.7$ (-, $\left.\mathrm{CH}_{2}\right), 26.8\left(-, \mathrm{CH}_{2}\right), 28.8\left(-, \mathrm{CH}_{2}\right), 30.0\left(-, \mathrm{CH}_{2}\right)$, $30.5\left(-, \mathrm{CH}_{2}\right), 33.1\left(-, \mathrm{CH}_{2}\right), 33.3\left(-, \mathrm{CH}_{2}\right), 40.5(+, \mathrm{CH}), 42.3(+$, $\mathrm{CH}), 51.2\left(+, \mathrm{CH}_{3}\right), 113.4(+, \mathrm{C}-2), 127.4\left(\mathrm{C}_{\text {quat }}\right), 132.1\left(+, \mathrm{C}-1^{\prime \prime}\right)$, $137.6\left(\mathrm{C}_{\text {quat }}\right), 145.6(+, \mathrm{C}-3), 151.6\left(\mathrm{C}_{\text {quat }}\right), 168.5\left(\mathrm{C}_{\text {quat }}, \mathrm{C}=\mathrm{O}\right) \mathrm{ppm}$. MS $(70 \mathrm{eV}): m / z(\%)=300(39)\left[\mathrm{M}^{+}\right], 294(2), 269(2)\left[\mathrm{M}^{+}-\mathrm{OCH}_{3}\right]$, 257 (1), 241 (32) $\left[\mathrm{M}^{+}-\mathrm{CO}_{2} \mathrm{CH}_{3}\right], 226$ (8), 192 (2), 159 (3), 145 (100), 131 (7), 117 (7), 91 (10), 67 (5), 55 (4), 41 (6). HRMS: calcd. for $\mathrm{C}_{20} \mathrm{H}_{28} \mathrm{O}_{2} 300.2091$ (correct HRMS).

tert-Butyl (E)-3-[2'-(5' -tert-Butoxy-4a' '-methyl-1" ${ }^{\prime \prime}, 2^{\prime \prime}, 3^{\prime \prime}, 4^{\prime \prime}$, $5^{\prime \prime}, 6^{\prime \prime}, 8 a^{\prime \prime}$-heptahydronaphth-2' '-enyl)cyclohex-1' -enyl]acrylate (23): According to GP 3, the bromobutadiene 18 (270 mg, $700 \mu \mathrm{mol})$ in $\mathrm{DMF} / \mathrm{MeCN} / \mathrm{H}_{2} \mathrm{O}(10: 5: 1)(5 \mathrm{~mL})$ was treated with the palladacycle $(52.5 \mathrm{mg}, 56 \mu \mathrm{mol}), n \mathrm{Bu}_{4} \mathrm{NOAc}(527 \mathrm{mg}$, $1.75 \mathrm{mmol})$ tert-butyl acrylate $(2.00 \mathrm{~mL})$ at $105^{\circ} \mathrm{C}$ for $4 \mathrm{~h}$. For complete consumption of the bromobutadiene 18 , a second portion of the palladacycle $(52.5 \mathrm{mg}, 56 \mu \mathrm{mol})$ was added and the mixture again heated at $105^{\circ} \mathrm{C}$ for $4 \mathrm{~h}$. Workup with diethyl ether $(40 \mathrm{~mL})$, water $(2 \times 30 \mathrm{~mL})$, extraction with diethyl ether $(40 \mathrm{~mL})$ and $\mathrm{FC}$ (34 $\mathrm{g}$ on silica gel, 20:1 v/v light petroleum/diethyl ether elution) gave compound $23(180 \mathrm{mg}, 60 \%)$ as a colorless wax. $R_{\mathrm{f}}=0.2$. IR (film): $\tilde{v}=2975 \mathrm{~cm}^{-1}, 2928,2857,1704,1614,1450,1389,1365$, 1309, 1293, 1274, 1247, 1192, 1148, 1076, 1050, 1019, 999, 984, 899, 881, 851. ${ }^{1} \mathrm{H}$ NMR $\left(250 \mathrm{MHz}, \mathrm{C}_{6} \mathrm{D}_{6}\right): \delta=0.72-0.93(\mathrm{~m}, 2 \mathrm{H})$, $1.07\left(\mathrm{~s}, 3 \mathrm{H}, \mathrm{CH}_{3}\right), 1.17$ [s, $\left.9 \mathrm{H}, \mathrm{C}\left(\mathrm{CH}_{3}\right)_{3}\right], 1.18-1.31(\mathrm{~m}, 4 \mathrm{H}), 1.43$ [s, $\left.9 \mathrm{H}, \mathrm{CO}_{2} \mathrm{C}\left(\mathrm{CH}_{3}\right)_{3}\right], 1.51-1.88(\mathrm{~m}, 7 \mathrm{H}), 1.90-2.19(\mathrm{~m}, 6 \mathrm{H}), 2.93$ (t, $\left.{ }^{3} J=5.8,{ }^{3} J=6.0 \mathrm{~Hz}, 1 \mathrm{H}, 5^{\prime \prime}-\mathrm{H}\right), 5.14\left(\mathrm{~d},{ }^{3} J=0.6 \mathrm{~Hz} 1 \mathrm{H}, 1^{\prime \prime}\right.$ H), $5.98\left(\mathrm{~d},{ }^{3} \mathrm{~J}=16.7 \mathrm{~Hz}, 1 \mathrm{H}, 2-\mathrm{H}\right) 8.11\left(\mathrm{~d},{ }^{3} \mathrm{~J}=16.7 \mathrm{~Hz}, 1 \mathrm{H}, 3-\right.$ H) ppm. ${ }^{13} \mathrm{C}$ NMR $\left(62.9 \mathrm{MHz}, \mathrm{C}_{6} \mathrm{D}_{6}\right.$, add. DEPT): $\delta=11.0(+$, $\left.\mathrm{CH}_{3}\right), 22.6\left(-, \mathrm{CH}_{2}\right), 22.8\left(-, \mathrm{CH}_{2}\right), 24.9\left(-, \mathrm{CH}_{2}\right), 25.4\left(-, \mathrm{CH}_{2}\right)$, $26.4\left(-, \mathrm{CH}_{2}\right), 27.1\left(-, \mathrm{CH}_{2}\right), 28.2\left[+, 3 \mathrm{C}, \mathrm{C}\left(\mathrm{CH}_{3}\right)_{3}\right], 29.2[+, 3 \mathrm{C}$, $\left.\mathrm{CO}_{2} \mathrm{C}\left(\mathrm{CH}_{3}\right)\right], 30.8\left(-, \mathrm{CH}_{2}\right), 31.0\left(-, \mathrm{CH}_{2}\right), 34.5\left(-, \mathrm{CH}_{2}\right), 37.7$ $\left(\mathrm{C}_{\text {quat }}, \mathrm{C}-4 \mathrm{a}^{\prime \prime}\right), 44.1\left(+, \mathrm{C}-8 \mathrm{a}^{\prime \prime}\right), 72.6\left[\mathrm{C}_{\text {quat }}, \mathrm{C}\left(\mathrm{CH}_{3}\right)_{3}\right], 77.5(+, \mathrm{C}-$ $\left.5^{\prime \prime}\right), 79.2\left[\mathrm{C}_{\text {quat }}, \mathrm{CO}_{2} \mathrm{C}\left(\mathrm{CH}_{3}\right)_{3}\right], 117.1(+, \mathrm{C}-2), 128.2\left(\mathrm{C}_{\text {quat }}\right), 130.2$ $\left(+, \mathrm{C}^{\prime} 7^{\prime \prime}\right), 137.7\left(\mathrm{C}_{\text {quat }}\right), 144.3(+, \mathrm{C}-3), 149.6\left(\mathrm{C}_{\text {quat }}\right), 167.0\left(\mathrm{C}_{\text {quat }}\right.$, $\mathrm{C}=\mathrm{O}) \mathrm{ppm}$. MS $(70 \mathrm{eV}): \mathrm{m} / \mathrm{z}(\%)=428(10)\left[\mathrm{M}^{+}\right], 372(51)\left[\mathrm{M}^{+}-\right.$ $\left.\mathrm{C}_{4} \mathrm{H}_{8}\right], 355$ (6), $316(18)\left[\mathrm{M}^{+}-2 \times \mathrm{C}_{4} \mathrm{H}_{8}\right], 315$ (32) $\left[\mathrm{M}^{+}-\mathrm{C}_{4} \mathrm{H}_{9}-\right.$ $\mathrm{C}_{4} \mathrm{H}_{8}$ ], 297 (36) $\left[\mathrm{M}^{+}-\mathrm{C}_{4} \mathrm{H}_{9}-\mathrm{C}_{4} \mathrm{H}_{8}-\mathrm{H}_{2} \mathrm{O}\right], 269$ (12), 253 (28), 231 (3), 205 (8), 185 (13), 171 (9), 145 (28), 105 (4), 95 (6), 84 (15), 57 (100) $\left[\mathrm{C}_{4} \mathrm{H}_{9}{ }^{+}\right], 41$ (20). HRMS: calcd. for $\mathrm{C}_{28} \mathrm{H}_{44} \mathrm{O}_{3} 428.3293$ (correct HRMS).

(E)-1,2-Bis[2-(tert-butyldimethylsilyl)vinyl]cyclohexene (24): ${ }^{1} \mathrm{H}$ NMR (300 MHz, $\left.\mathrm{CDCl}_{3}\right): \delta=-0.03\left[\mathrm{~s}, 12 \mathrm{H}, \mathrm{Si}\left(\mathrm{CH}_{3}\right)_{2}\right], 0.83[\mathrm{~s}, 18$ $\left.\mathrm{H}, \mathrm{C}\left(\mathrm{CH}_{3}\right)_{3}\right], 1.60\left[\mathrm{~m}_{\mathrm{C}}, 4 \mathrm{H}, 4^{\prime}\left(5^{\prime}\right)-\mathrm{H}\right], 2.27\left[\mathrm{~m}_{\mathrm{c}}, 4 \mathrm{H}, 6^{\prime}\left(3^{\prime}\right)-\mathrm{H}\right]$, $5.83\left(\mathrm{~d},{ }^{3} \mathrm{~J}=18.9 \mathrm{~Hz}, 2 \mathrm{H}, 1-\mathrm{H}\right), 7.29\left(\mathrm{~d},{ }^{3} \mathrm{~J}=18.9 \mathrm{~Hz}, 2 \mathrm{H}, 2-\mathrm{H}\right)$ ppm. MS (ESI, MeOH): $m / z(\%)=599(10), 569$ (12), 455 (10), 363 (40) $\left[\mathrm{M}+\mathrm{H}^{+}\right], 2291$ (22), 289 (15), 235 (23), 231 (10), 179 (16), 146 (9), 115 (20), 102 (100), 74 (14).

tert-Butyl 3-(tert-Butyldimethylsilyl)-2,3,5,6,7,8-hexahydronaphthalene-2-carboxylate (26), tert-Butyl 3-(tert-Butyldimethylsilyl)- 1,2,5,6,7,8-hexahydronaphthalene-2-carboxylate (27): According to GP 5, the hexatriene $19(70.0 \mathrm{mg}, 0.201 \mathrm{mmol})$ in decalin $(2.00 \mathrm{~mL})$ was heated at $205^{\circ} \mathrm{C}$ for $45 \mathrm{~min}$. FC ( $25 \mathrm{~g}$ of silica gel, $20: 1 \mathrm{v} / \mathrm{v}$ pentane/diethyl ether elution) yielded a mixture of diene 26 and diene 27 in a ratio of 1:1.9 (according to ${ }^{1} \mathrm{H}$ NMR) as a colorless wax $(55.3 \mathrm{mg}, 79 \%) . R_{\mathrm{f}}=0.3 .{ }^{1} \mathrm{H} \mathrm{NMR}\left(300 \mathrm{MHz}, \mathrm{CDCl}_{3}\right.$, signals which can be assigned to the minor product $\mathbf{2 6}$ are marked with $\left.{ }^{\#}\right): \delta=0.05\left[\mathrm{~s}, 6 \mathrm{H}, \mathrm{Si}\left(\mathrm{CH}_{3}\right)_{2}\right], 0.08\left[\mathrm{~s}, 6 \mathrm{H}, \mathrm{Si}\left(\mathrm{CH}_{3}\right)_{2}\right]^{\#}, 0.85[\mathrm{~s}, 9$ $\left.\mathrm{H}, \mathrm{SiC}\left(\mathrm{CH}_{3}\right)_{3}\right], 0.90\left[\mathrm{~s}, 9 \mathrm{H}, \mathrm{SiC}\left(\mathrm{CH}_{3}\right)_{3}\right]^{\#}, 1.18\left[\mathrm{~s}, 9 \mathrm{H}, \mathrm{CO}_{2} \mathrm{C}-\right.$ $\left.\left(\mathrm{CH}_{3}\right)_{3}\right], 1.38\left[\mathrm{~s}, 9 \mathrm{H}, \mathrm{CO}_{2} \mathrm{C}\left(\mathrm{CH}_{3}\right)_{3}\right]^{\#}, 1.77-1.89(\mathrm{~m}, 4 \mathrm{H}), 1.92-2.17$ (m, $4 \mathrm{H}), 2.22-2.37(\mathrm{~m}, 2 \mathrm{H}), 2.48\left(\mathrm{~m}_{\mathrm{c}}, 1 \mathrm{H}\right), 2.72-2.83(\mathrm{~m}, 3 \mathrm{H})$, $2.98\left(\mathrm{~m}_{\mathrm{c}}, 1 \mathrm{H}\right), 6.08(\mathrm{~s}, 1 \mathrm{H}), 6.62(\mathrm{~s}, 1 \mathrm{H})^{\#} \mathrm{ppm}$.

[3-(tert-Butyldimethylsilyl)-1,2,5,6,7,8-hexahydronaphthalen-2-yl]methanol (28): According to GP 5, the hexatriene $29(60.0 \mathrm{mg}$, $0.215 \mathrm{mmol})$ in decalin $(0.60 \mathrm{~mL})$ was heated at $205^{\circ} \mathrm{C}$ for $1.75 \mathrm{~h}$. FC (15 g of silica gel, 2:1 v/v pentane/diethyl ether elution) yielded compound $28(52.7 \mathrm{mg}, 88 \%)$ as a colorless wax. $R_{\mathrm{f}}=0.4$. IR (film): $\tilde{v}=3306 \mathrm{~cm}^{-1}, 2951,2927,2856,1655,1566,1470,1462$, 1437, 1360, 1315, 1247, 1187, 1147, 1050, 1024, 937, 864, 825, 809, 767. ${ }^{1} \mathrm{H}$ NMR $\left(300 \mathrm{MHz}, \mathrm{CDCl}_{3}\right): \delta=0.05\left[\mathrm{~s}, 3 \mathrm{H}, \mathrm{Si}\left(\mathrm{CH}_{3}\right)_{2}\right], 0.07$ [s, $\left.3 \mathrm{H}, \mathrm{Si}\left(\mathrm{CH}_{3}\right)_{2}\right], 0.89$ [s, $\left.9 \mathrm{H}, \mathrm{C}\left(\mathrm{CH}_{3}\right)_{3}\right], 1.24-1.40(\mathrm{~m}, 1 \mathrm{H}), 1.49$ $1.74\left[\mathrm{~m}, 4 \mathrm{H}, 4^{\prime}\left(5^{\prime}\right)-\mathrm{H}\right], 1.88-2.20(\mathrm{~m}, 6 \mathrm{H}), 2.22-2.48(\mathrm{~m}, 1 \mathrm{H}, 2-$ $\mathrm{H}), 3.37\left(\mathrm{~d},{ }^{3} \mathrm{~J}=6.3 \mathrm{~Hz}, 2 \mathrm{H}, \mathrm{CH}_{2} \mathrm{O}\right), 6.02(\mathrm{~s}, 1 \mathrm{H}, 4-\mathrm{H}) \mathrm{ppm} .{ }^{13} \mathrm{C}$ NMR (75.6 MHz, $\mathrm{CDCl}_{3}$, add. APT): $\delta=-6.5\left[+, \mathrm{CH}_{3}, \mathrm{Si}-\right.$ $\left.\left(\mathrm{CH}_{3}\right)_{2}\right],-6.2\left[+, \mathrm{CH}_{3}, \mathrm{Si}\left(\mathrm{CH}_{3}\right)_{2}\right], 17.2\left[-, \mathrm{C}_{\text {quat }}, \mathrm{C}\left(\mathrm{CH}_{3}\right)_{3}\right], 22.7$ (-, $\left.\mathrm{CH}_{2}\right), 23.2\left(-, \mathrm{CH}_{2}\right), 26.9\left[+, 3 \mathrm{C}, \mathrm{CH}_{3}, \mathrm{C}\left(\mathrm{CH}_{3}\right)_{3}\right], 27.8\left(-, \mathrm{CH}_{2}\right)$, $30.0\left(-, \mathrm{CH}_{2}\right), 30.6\left(-, \mathrm{CH}_{2}\right), 38.0(+, \mathrm{CH}, \mathrm{C}-2), 62.4\left(-, \mathrm{CH}_{2}\right.$, $\left.\mathrm{CH}_{2} \mathrm{OH}\right), 126.7$ (-, $\left.\mathrm{C}_{\text {quat }}\right), 130.3$ (-, $\left.\mathrm{C}_{\text {quat }}\right), 132.1$ (-, $\left.\mathrm{C}_{\text {quat }}\right), 140.2$ $(+, \mathrm{CH}) \mathrm{ppm}$. MS $(70 \mathrm{eV}): m / z(\%)=278(3)\left[\mathrm{M}^{+}\right], 221(27)\left[\mathrm{M}^{+}{ }_{-}\right.$ $\mathrm{C}_{4} \mathrm{H}_{9}$ ], 203 (20), 189 (6), 161 (4), 145 (68), 131 (17), 118 (11), 104 (8), 91 (17), 75 (100), 73 (43), 61 (8), 59 (19). HRMS: calcd. for $\mathrm{C}_{17} \mathrm{H}_{30} \mathrm{OSi} 278.2068$ (correct HRMS).

[3-(Trimethylsilyl)-1,2,5,6,7,8-hexahydronaphthalene-2-yl]methanol (28-SiMe $)$ : According to GP 5, the hexatriene 29-SiMe $(15 \mathrm{mg}$, $0.063 \mathrm{mmol})$ in decalin $(1.0 \mathrm{~mL})$ was heated at $205^{\circ} \mathrm{C}$ for $1.75 \mathrm{~h}$. FC (10 g of silica, 2:1 v/v pentane/diethyl ether elution) yielded 28$\mathrm{SiMe}_{3}(5.0 \mathrm{mg}, 33 \%)$ as a colorless wax. $R_{\mathrm{f}}=0.4$. IR (film): $\tilde{v}=$ $3356 \mathrm{~cm}^{-1}, 2929,2858,1673,1570,1447,1438,1309,1247,1150$, 1024, 998, 929, 866, 836, 752. ${ }^{1} \mathrm{H}$ NMR $\left(300 \mathrm{MHz}, \mathrm{CDCl}_{3}\right): \delta=$ 0.19 [s, $\left.9 \mathrm{H}, \mathrm{Si}\left(\mathrm{CH}_{3}\right)_{3}\right], 1.28-1.72(\mathrm{~m}, 1 \mathrm{H}), 1.89-2.15(\mathrm{~m}, 3 \mathrm{H})$, 2.23-2.39 (m, $6 \mathrm{H}), 2.40-2.50(\mathrm{~m}, 1 \mathrm{H}, 2-\mathrm{H}), 3.36-3.53(\mathrm{~m}, 2 \mathrm{H}$, $\left.\mathrm{CH}_{2} \mathrm{O}\right), 6.19(\mathrm{~s}, 1 \mathrm{H}, 4-\mathrm{H}) \mathrm{ppm}$. MS $(70 \mathrm{eV}): m / z(\%)=236(26)$ $\left[\mathrm{M}^{+}\right], 219(8)\left[\mathrm{M}^{+}-\mathrm{OH}\right], 205(8)\left[\mathrm{M}^{+}-\mathrm{CH}_{2} \mathrm{OH}\right], 204$ (6) $\left[\mathrm{M}^{+}{ }_{-}\right.$ $\mathrm{CH}_{3} \mathrm{OH}$ ], 189 (14), 146 (17), 145 (22), 132 (15), 131 (36), 129 (11), 118 (24), 117 (13), 105 (16), 104 (18), 103 (7), 91 (31), 75 (42), $73(100)\left[\mathrm{Si}\left(\mathrm{CH}_{3}\right)^{+}\right], 61$ (6), 59 (12). HRMS: calcd. for $\mathrm{C}_{14} \mathrm{H}_{24} \mathrm{OSi}$ 236.1598 (correct HRMS).

(E)-3-\{2'-[(E)-2' '-(tert-Butyldimethylsilyl)vinyl]cyclohex-1' enyl\}prop-2-en-1-ol (29): According to GP 4, the hexatriene 19 $(100 \mathrm{mg}, 0.287 \mathrm{mmol})$ in toluene $(5.00 \mathrm{~mL})$, DIBALH $(2.30 \mathrm{~mL}$, $2.30 \mathrm{mmol})$, workup with diethyl ether $(50 \mathrm{~mL}), \mathrm{KHSO}_{4}$ solution $(20 \mathrm{~mL})$, extraction with diethyl ether $(20 \mathrm{~mL}), \mathrm{NaHCO}_{3}$ solution $(20 \mathrm{~mL})$, water $(20 \mathrm{~mL})$ and FC (20 g of silica gel, $2: 1 \mathrm{v} / \mathrm{v}$ pentane/ diethyl ether elution) gave compound $29(64.7 \mathrm{mg}, 81 \%)$ as a colorless oil. $R_{\mathrm{f}}=0.4$. IR (film): $\tilde{v}=3398 \mathrm{~cm}^{-1}, 2928,2883,2856,1665$, 1499, 1463, 1445, 1409, 1389, 1361, 1252, 1154, 1048, 1007, 987, 938, 834, 832, 810, 777. ${ }^{1} \mathrm{H}$ NMR $\left(300 \mathrm{MHz}, \mathrm{CDCl}_{3}\right): \delta=0.07[\mathrm{~s}$, $\left.6 \mathrm{H}, \mathrm{Si}\left(\mathrm{CH}_{3}\right)_{2}\right], 0.88\left[\mathrm{~s}, 9 \mathrm{H}, \mathrm{C}\left(\mathrm{CH}_{3}\right)_{3}\right], 1.61-1.70\left[\mathrm{~m}, 4 \mathrm{H}, 4^{\prime}\left(5^{\prime}\right)-\right.$ $\mathrm{H}$ ], 2.22-2.37 [m, $\left.4 \mathrm{H}, 3^{\prime}\left(6^{\prime}\right)-\mathrm{H}\right], 4.26\left(\mathrm{dd},{ }^{3} J_{1}=6.0,{ }^{3} J_{2}=0.8 \mathrm{~Hz}\right.$, $2 \mathrm{H}, 1-\mathrm{H}), 4.87\left(\mathrm{dt},{ }^{3} J_{1}=15.5,{ }^{3} J_{2}=6.0 \mathrm{~Hz}, 1 \mathrm{H}, 2-\mathrm{H}\right), 5.91\left(\mathrm{~d},{ }^{3} J\right.$ $\left.=19.0 \mathrm{~Hz}, 1 \mathrm{H}, 1^{\prime \prime}-\mathrm{H}\right), 7.00\left(\mathrm{~d},{ }^{3} J=15.4 \mathrm{~Hz}, 1 \mathrm{H}, 3-\mathrm{H}\right), 7.24(\mathrm{~d}$, $\left.{ }^{3} J=19.0 \mathrm{~Hz}, 1 \mathrm{H}, 2^{\prime \prime}-\mathrm{H}\right) \mathrm{ppm} .{ }^{13} \mathrm{C}$ NMR $\left(75.6 \mathrm{MHz}, \mathrm{CDCl}_{3}\right.$, add. 
APT): $\delta=-6.01\left[+, 2 \mathrm{C}, \mathrm{CH}_{3}, \mathrm{Si}\left(\mathrm{CH}_{3}\right)_{2}\right], 16.63\left[-, \mathrm{C}_{\mathrm{quat}}, C-\right.$ $\left.\left(\mathrm{CH}_{3}\right)_{3}\right], 22.44\left(-, \mathrm{CH}_{2}\right), 22.47\left(-, \mathrm{CH}_{2}\right), 26.14\left(-, \mathrm{CH}_{2}\right), 26.50[+$, $\left.3 \mathrm{C}, \mathrm{CH}_{3}, \mathrm{C}\left(\mathrm{CH}_{3}\right)_{3}\right], 26.67\left(-, \mathrm{CH}_{2}\right), 64.40\left(-, \mathrm{CH}_{2}, \mathrm{C}-1\right), 125.59$ $(+, \mathrm{CH}), 127.20(+, \mathrm{CH}), 129.02(+, \mathrm{CH}), 132.25\left(-, \mathrm{C}_{\text {quat }}\right), 134.43$ $\left(-, \mathrm{C}_{\text {quat }}\right), 141.64(+, \mathrm{CH}) \mathrm{ppm}$. MS $(70 \mathrm{eV}): m / z(\%)=278(3)$ $\left[\mathrm{M}^{+}\right], 203$ (6), 145 (17), 131 (7), 115 (13), 105 (3), 91 (8), 75 (100), 59 (13), 43 (7). HRMS: calcd. for $\mathrm{C}_{17} \mathrm{H}_{30} \mathrm{OSi} 278.2068$ (correct HRMS).

tert-Butyldimethyl[3-(tetrahydropyran-2-yloxymethyl)-3,4,5,6,7,8hexahydronaphthalen-2-yl|silane (30): According to GP 5, the hexatriene $31(81.0 \mathrm{mg}, 0.223 \mathrm{mmol})$ in decalin $(3.00 \mathrm{~mL})$ was heated at $205^{\circ} \mathrm{C}$ for $2 \mathrm{~h}$. FC (13 g of silica gel, $10: 1 \mathrm{v} / \mathrm{v}$ pentane/diethyl ether elution) yielded compound $\mathbf{3 0}(75.2 \mathrm{mg}, 93 \%)$ as a colorless wax. The diastereomeric ratio was found to be $1: 1 . R_{\mathrm{f}}=0.5$. IR (film): $\tilde{v}=2926 \mathrm{~cm}^{-1}, 2856,2737,1655,1566,1463,1440,1410,1387$, 1360, 1351, 1322, 1257, 1200, 1189, 1120, 1077, 1056, 1030, 975, 936, 907, 868, 825. ${ }^{1} \mathrm{H}$ NMR (300 MHz, $\mathrm{CDCl}_{3}$ distinguishable signals of the diastereomers are marked with $\left.{ }^{\#}\right): \delta=0.07[\mathrm{~s}, 3 \mathrm{H}$, $\mathrm{Si}\left(\mathrm{CH}_{3}\right)_{2}$ ], 0.09 [s, $\left.3 \mathrm{H}, \mathrm{Si}\left(\mathrm{CH}_{3}\right)_{2}\right], 0.89$ [s, $\left.9 \mathrm{H}, \mathrm{C}\left(\mathrm{CH}_{3}\right)_{3}\right], 1.21-1.40$ (m, 1 H), 1.41-1.91 (m, 11 H), 1.92-2.34 (m, 4 H), 2.48-2.61 (m, 1 H, 3-H), 3.03-3.19 (m, $1 \mathrm{H}), 3.36-3.58(\mathrm{~m}, 1 \mathrm{H}), 3.70-3.95$ (m, 1 $\mathrm{H}, \mathrm{OCHO}), 4.45\left(\mathrm{~m}_{\mathrm{c}}, 1 \mathrm{H}, 3-\mathrm{CH}_{2} \mathrm{O}\right), 4.59\left(\mathrm{~m}_{\mathrm{c}}, 1 \mathrm{H}, 3-\mathrm{CH}_{2} \mathrm{O}\right), 5.99$ $\left(\mathrm{d},{ }^{3} \mathrm{~J}=2.5 \mathrm{~Hz}, 1 \mathrm{H}, 1-\mathrm{H}\right) \mathrm{ppm} .{ }^{13} \mathrm{C} \mathrm{NMR}\left(75.6 \mathrm{MHz}, \mathrm{CDCl}_{3}\right.$, add. APT $): \delta=-6.5\left[+, \mathrm{CH}_{3}, \mathrm{Si}\left(\mathrm{CH}_{3}\right)_{2}\right],-6.4\left[+, \mathrm{CH}_{3}, \mathrm{Si}\left(\mathrm{CH}_{3}\right)_{2}\right]$, $17.3\left[-, \mathrm{C}_{\text {quat }}, C\left(\mathrm{CH}_{3}\right)_{3}\right], 19.5\left(-, \mathrm{CH}_{2}\right), 20.0\left(-, \mathrm{CH}_{2}\right), 23.1\left(-, \mathrm{CH}_{2}\right)$, $23.4\left(-, \mathrm{CH}_{2}\right)^{\#}, 25.7\left(-, \mathrm{CH}_{2}\right), 25.8\left(-, \mathrm{CH}_{2}\right)^{\#}, 27.1\left[+, 3 \mathrm{C}, \mathrm{CH}_{3}\right.$, $\left.\mathrm{C}\left(\mathrm{CH}_{3}\right)_{3}\right], 28.1\left(-, \mathrm{CH}_{2}\right), 28.1\left(-, \mathrm{CH}_{2}\right)^{\#}, 30.1\left(-, \mathrm{CH}_{2}\right), 30.2(-$, $\left.\mathrm{CH}_{2}\right)^{\#}, 30.8\left(-, \mathrm{CH}_{2}\right), 30.9\left(-, \mathrm{CH}_{2}\right), 35.4(+, \mathrm{CH}, \mathrm{C}-3), 36.2(+$, $\mathrm{CH}, \mathrm{C}-3)^{\#}, 61.7\left(-, \mathrm{CH}_{2}\right), 62.6\left(-, \mathrm{CH}_{2}\right)^{\#}, 65.4\left(-, \mathrm{CH}_{2}\right), 66.9(-$, $\left.\mathrm{CH}_{2}\right)^{\#}, 97.8(+, \mathrm{CH}), 100.3(+, \mathrm{CH})^{\#}, 126.9\left(-, \mathrm{C}_{\text {quat }}\right), 130.1(-$, $\left.\mathrm{C}_{\text {quat }}\right), 130.3\left(-, \mathrm{C}_{\text {quat }}\right)^{\#}, 132.5\left(-, \mathrm{C}_{\text {quat }}\right), 132.8\left(-, \mathrm{C}_{\text {quat }}\right)^{\#}, 140.2$ $(+, \mathrm{CH}), 140.3(+, \mathrm{CH})^{\#}$ ppm. MS $(70 \mathrm{eV}): m / z(\%)=362(3)\left[\mathrm{M}^{+}\right]$, 305 (12) $\left[\mathrm{M}^{+}-\mathrm{C}_{4} \mathrm{H}_{9}\right], 262$ (10), 221 (31), 219 (7), 205 (20), 203 (27), 189 (23), 159 (6), 146 (18), 145 (37), 131 (15), 118 (7), 115 (18), 103 (9), 89 (5), 85 (100), 73 (60), 67 (17), $57(22)\left[\mathrm{C}_{4} \mathrm{H}_{9}^{+}\right]$. HRMS: calcd. for $\mathrm{C}_{22} \mathrm{H}_{38} \mathrm{O}_{2} \mathrm{Si} 362.2643$ (correct HRMS).

(E)-tert-Butyldimethyl(2-\{(E)-2'-[3' -(tetrahydropyran-2-yloxy)propenyl]cyclohex-1' -enyl\}vinyl)silane (31): To a solution of the hexatriene 29 (140 mg, $0.485 \mathrm{mmol}$ ) and 3,4-dihydro- $2 H$-pyran $(81.6 \mathrm{mg}$, $0.970 \mathrm{mmol})$ in ethyl acetate $(3.00 \mathrm{~mL})$ was added at ambient temperature scandium(III) trifluoromethanesulfonate $(3.0 \mathrm{mg}$, $0.0060 \mathrm{mmol}$ ), and the mixture was stirred for $1 \mathrm{~h}$. It was poured into diethyl ether $(50 \mathrm{~mL})$, and the mixture washed with satd. $\mathrm{NaHCO}_{3}$ solution $(20 \mathrm{~mL})$. After extraction of the combined aqueous phases with diethyl ether $(2 \times 20 \mathrm{~mL})$, the combined organic layers were dried with $\mathrm{MgSO}_{4}$ and concentrated in vacuo. FC (24 g of silica gel, 10:1 v/v pentane/diethyl ether elution) yielded compound $31(171 \mathrm{mg}, 97 \%)$ as a colorless oil. $R_{\mathrm{f}}=0.3$. IR (film): $\tilde{\mathrm{v}}=$ $2926 \mathrm{~cm}^{-1}, 2854,1591,1557,1462,1445,1360,1247,1200,1182$, 1130, 1077, 1023, 980, 905, 867, 827. ${ }^{1} \mathrm{H}$ NMR (300 MHz, $\left.\mathrm{CDCl}_{3}\right)$ : $\delta=0.06\left[\mathrm{~s}, 6 \mathrm{H}, \mathrm{Si}\left(\mathrm{CH}_{3}\right)_{2}\right], 0.89\left[\mathrm{~s}, 9 \mathrm{H}, \mathrm{C}\left(\mathrm{CH}_{3}\right)_{3}\right], 1.20-1.39(\mathrm{~m}$, $4 \mathrm{H}), 1.47-1.92(\mathrm{~m}, 6 \mathrm{H}), 2.21-2.36(\mathrm{~m}, 4 \mathrm{H}), 3.45-3.62(\mathrm{~m}, 1 \mathrm{H})$, 3.82-3.98 (m, $1 \mathrm{H}), 4.00-4.18(\mathrm{~m}, 1 \mathrm{H}), 4.23-4.43(\mathrm{~m}, 1 \mathrm{H}), 4.63-$ $4.76(\mathrm{~m}, 1 \mathrm{H}), 5.82\left(\mathrm{dt},{ }^{3} J_{1}=15.1,{ }^{3} J_{2}=5.5 \mathrm{~Hz}, 1 \mathrm{H}, 2^{\prime \prime}-\mathrm{H}\right), 5.89$ $\left(\mathrm{dt},{ }^{3} J=18.7 \mathrm{~Hz}, 1 \mathrm{H}, 1-\mathrm{H}\right), 7.05\left(\mathrm{~d},{ }^{3} J=16.5 \mathrm{~Hz}, 1 \mathrm{H}, 3^{\prime \prime}-\mathrm{H}\right)$, $7.27\left(\mathrm{~d},{ }^{3} J=18.9 \mathrm{~Hz}, 1 \mathrm{H}, 2-\mathrm{H}\right) \mathrm{ppm} .{ }^{13} \mathrm{C} \mathrm{NMR}\left(75.6 \mathrm{MHz}, \mathrm{C}_{6} \mathrm{D}_{6}\right.$, add. APT): $\delta=-5.9\left[+, 2 \mathrm{C}, \mathrm{CH}_{3}, \mathrm{Si}\left(\mathrm{CH}_{3}\right)_{2}\right], 16.9\left[-, \mathrm{C}_{\text {quat }}, C\right.$ $\left.\left(\mathrm{CH}_{3}\right)_{3}\right], 19.4\left(-, \mathrm{CH}_{2}\right), 22.7\left(-, \mathrm{CH}_{2}\right), 22.7\left(-, \mathrm{CH}_{2}\right), 25.8\left(-, \mathrm{CH}_{2}\right)$, $26.3\left(-, \mathrm{CH}_{2}\right), 26.6\left[+, 3 \mathrm{C}, \mathrm{CH}_{3}, \mathrm{C}\left(\mathrm{CH}_{3}\right)_{3}\right], 26.8\left(-, \mathrm{CH}_{2}\right), 30.9$ (-, $\left.\mathrm{CH}_{2}\right), 61.3\left(-, \mathrm{CH}_{2}, \mathrm{C}-1^{\prime \prime}\right), 67.8\left(-, \mathrm{CH}_{2}\right), 97.5$ (+, $\left.\mathrm{CH}, \mathrm{OCHO}\right)$, $124.6(+, \mathrm{CH}), 126.0(+, \mathrm{CH}), 128.8(+, \mathrm{CH}), 133.2\left(-, \mathrm{C}_{\text {quat }}\right), 133.7$ $\left(-, \mathrm{C}_{\text {quat }}\right), 142.8(+, \mathrm{CH})$ ppm. MS $(70 \mathrm{eV}): m / z(\%)=362(10)$ $\left[\mathrm{M}^{+}\right], 278$ (2), 261 (14), 247 (6), 221 (3), 205 (14), 203 (17), 177 (7),
159 (69), 141 (27), 117 (17), 101 (12), 91 (15), 85 (91), 75 (100), 59 (41), 55 (16). HRMS: calcd. for $\mathrm{C}_{22} \mathrm{H}_{38} \mathrm{O}_{2} \mathrm{Si} 362.2643$ (correct HRMS).

tert-Butyl 2,3,4a,5,7,8,9,10-Octahydronaphtho[1,2-b][1,4]dioxine-5carboxylate (32) and tert-Butyl 2,3,5,6,7,8,9,10-Octahydronaphtho[1,2-b][1,4]dioxine-5-carboxylate (33): According to GP 5, the hexatriene $20(32.0 \mathrm{mg}, 0.109 \mathrm{mmol})$ in decalin $(0.50 \mathrm{~mL})$ was heated at $205^{\circ} \mathrm{C}$ for $45 \mathrm{~min}$. After FC ( $20 \mathrm{~g}$ of silica gel, 5:1 v/v pentane/diethyl ether elution), a mixture of compounds 32 and 33 in a ratio of 1:3.1 (according to ${ }^{1} \mathrm{H}$ NMR) was obtained as a colorless wax (23.0 mg, $72 \%) . R_{\mathrm{f}}=0.4$. IR (film): $\tilde{v}=2976 \mathrm{~cm}^{-1}, 2933$, 2877, 1723, 1638, 1612, 1578, 1481, 1457, 1437, 1392, 1368, 1327, 1308, 1245, 1156, 1091, 1067, 988, 966, 919, 848. ${ }^{1}$ H NMR $\left(300 \mathrm{MHz}, \mathrm{CDCl}_{3}\right.$, signals which can be assigned to the minor product 32 are marked with $\left.{ }^{\#}\right): \delta=1.42\left[\mathrm{~s}, 9 \mathrm{H}, \mathrm{CO}_{2} \mathrm{C}\left(\mathrm{CH}_{3}\right)_{3}\right], 1.44$ $\left[\mathrm{s}, 9 \mathrm{H}, \mathrm{CO}_{2} \mathrm{C}\left(\mathrm{CH}_{3}\right)_{3}\right]^{\#}, 1.51-1.73(\mathrm{~m}, 4 \mathrm{H}), 1.75-1.78(\mathrm{~m}, 4 \mathrm{H})^{\#}$, $1.91-2.23(\mathrm{~m}, 6 \mathrm{H}), 2.25-2.55(\mathrm{~m}, 2 \mathrm{H}), 3.04\left(\mathrm{dd},{ }^{3} J_{1}=8.5,{ }^{3} J_{2}=\right.$ $5.8 \mathrm{~Hz}, 1 \mathrm{H}), 3.31\left(\mathrm{dd},{ }^{3} J_{1}=11.3,{ }^{3} J_{2}=5.2 \mathrm{~Hz}, 1 \mathrm{H}\right)^{\#}, 3.93-4.24$ $\left(\mathrm{m}_{\mathrm{c}}, 4 \mathrm{H}\right), 5.74\left(\mathrm{~m}_{\mathrm{c}}, 1 \mathrm{H}\right) \mathrm{ppm} .{ }^{13} \mathrm{C} \mathrm{NMR}\left(75.6 \mathrm{MHz}, \mathrm{CDCl}_{3}\right.$, add. APT): $\delta=22.0\left(-, \mathrm{CH}_{2}\right), 22.1\left(-, \mathrm{CH}_{2}\right), 22.3\left(-, \mathrm{CH}_{2}\right), 22.6(-$, $\left.\mathrm{CH}_{2}\right), 22.6\left(-, \mathrm{CH}_{2}\right), 22.8\left(-, \mathrm{CH}_{2}\right), 25.4\left(-, \mathrm{CH}_{2}\right), 28.2[+, 3 \mathrm{C}$, $\left.\mathrm{CO}_{2} \mathrm{C}\left(\mathrm{CH}_{3}\right)_{3}\right], 28.3\left[+, 3 \mathrm{C}, \mathrm{CO}_{2} \mathrm{C}\left(\mathrm{CH}_{3}\right)_{3}\right]^{\#}, 64.4\left(-, \mathrm{CH}_{2}\right), 64.8$ $\left(-, \mathrm{CH}_{2}\right), 65.0\left(-, \mathrm{CH}_{2}\right), 66.1\left(-, \mathrm{CH}_{2}\right), 67.4\left(-, \mathrm{CH}_{2}\right), 80.7\left[\mathrm{C}_{\text {quat }}\right.$, $\left.\mathrm{CO}_{2} \mathrm{C}\left(\mathrm{CH}_{3}\right)_{3}\right], 80.9\left[\mathrm{C}_{\text {quat }}, \mathrm{CO}_{2} \mathrm{C}\left(\mathrm{CH}_{3}\right)_{3}\right], 116.6(+, \mathrm{CH}), 123.8(-$, $\left.\mathrm{C}_{\text {quat }}\right), 124.3$ (-, $\left.\mathrm{C}_{\text {quat }}\right), 128.1$ (-, $\left.\mathrm{C}_{\text {quat }}\right), 128.1$ (-, $\left.\mathrm{C}_{\text {quat }}\right), 131.3$ (-, $\left.\mathrm{C}_{\text {quat }}\right), 131.3\left(-, \mathrm{C}_{\text {quat }}\right), 131.9\left(-, \mathrm{C}_{\text {quat }}\right), 132.6\left(-, \mathrm{C}_{\text {quat }}\right), 172.7(-$, $\left.\mathrm{C}_{\text {quat }}, \mathrm{C}=\mathrm{O}\right), 173.1\left(-, \mathrm{C}_{\text {quat }}, \mathrm{C}=\mathrm{O}\right)^{\#}$ ppm. MS $(70 \mathrm{eV}): \mathrm{m} / \mathrm{z}(\%)=$ $292(50)\left[\mathrm{M}^{+}\right], 290(22), 236(65)\left[\mathrm{M}^{+}-\mathrm{C}_{4} \mathrm{H}_{8}\right], 234$ (58), 217 (18), 206 (10), 191 (100), 190 (53), 162 (23), 149 (49), 134 (11), 122 (47), 107 (11), 94 (14), 91 (25), 86 (69), 84 (87), 79 (28), 77 (19), 73 (17), 65 (7), 57 (52) $\left[\mathrm{C}_{4} \mathrm{H}_{9}^{+}\right], 55$ (17). HRMS: calcd. for $\mathrm{C}_{17} \mathrm{H}_{24} \mathrm{O}_{4}$ 292.1676 (correct HRMS).

5-Methoxymethoxymethyl-2,3,5,6,7,8,9,10-octahydronaphtho[1,2-b][1,4]dioxine (34): According to GP 5, the hexatriene 36 (36.3 mg, $0.136 \mathrm{mmol})$ in decalin $(1.0 \mathrm{~mL})$ was heated at $150^{\circ} \mathrm{C}$ for $12 \mathrm{~h}$. FC (10 $\mathrm{g}$ of silica gel, 2:1 v/v pentane/diethyl ether elution) yielded compound $34(33.1 \mathrm{mg}, 91 \%)$ as a colorless wax. $R_{\mathrm{f}}=0.6$. IR (film): $\tilde{v}=2928 \mathrm{~cm}^{-1}, 2882(\mathrm{C}-\mathrm{H}), 1681,1632,1491,1441,1381$, 1333, 1292, 1277, 1242, 1201, 1175, 1150, 1109, 1075, 1041, 944, $918,799 \mathrm{~cm}^{-1} .{ }^{1} \mathrm{H}$ NMR $\left(300 \mathrm{MHz}, \mathrm{CDCl}_{3}\right): \delta=1.47-1.68[\mathrm{~m}, 4$ H, 8(9)-H], 1.92-2.00 (m, 2 H, 7-H), 2.01-2.07 (m, 2 H, 10-H), 2.08-2.17 (m, $1 \mathrm{H}, 6-\mathrm{H}), 2.28-2.41(\mathrm{~m}, 1 \mathrm{H}, 6-\mathrm{H}), 2.53\left(\mathrm{~h},{ }^{3} \mathrm{~J}=\right.$ $4.4 \mathrm{~Hz}, 1 \mathrm{H}, 5-\mathrm{H}), 3.35\left(\mathrm{~s}, 3 \mathrm{H}, \mathrm{OCH}_{3}\right), 3.40\left(\mathrm{dd},{ }^{3} J_{1}=18.4,{ }^{3} J_{2}=\right.$ $\left.9.1 \mathrm{~Hz}, 1 \mathrm{H}, \mathrm{CH}_{2} \mathrm{O}\right), 3.52\left(\mathrm{dd},{ }^{3} J_{1}=9.2,{ }^{3} J_{2}=4.7 \mathrm{~Hz}, 1 \mathrm{H}, \mathrm{CH}_{2} \mathrm{O}\right)$, $3.95-4.12[\mathrm{~m}, 4 \mathrm{H}, 2(3)-\mathrm{H}], 4.60\left(\mathrm{~d},{ }^{3} J=6.6 \mathrm{~Hz}, 1 \mathrm{H}, \mathrm{OCH}_{2} \mathrm{O}\right)$, $4.63\left(\mathrm{~d},{ }^{3} \mathrm{~J}=6.6 \mathrm{~Hz}, 1 \mathrm{H}, \mathrm{OCH}_{2} \mathrm{O}\right) \mathrm{ppm} .{ }^{13} \mathrm{C} \mathrm{NMR}(75.6 \mathrm{MHz}$, $\mathrm{CDCl}_{3}$, add. APT): $\delta=22.6\left(-, \mathrm{CH}_{2}\right), 22.7\left(-, \mathrm{CH}_{2}\right), 22.9\left(-, \mathrm{CH}_{2}\right)$, $30.2\left(-, \mathrm{CH}_{2}\right), 32.0\left(-, \mathrm{CH}_{2}\right), 36.7(+, \mathrm{CH}, \mathrm{C}-5), 55.3\left(+, \mathrm{CH}_{3}\right.$, $\left.\mathrm{OCH}_{3}\right), 64.7\left(-, \mathrm{CH}_{2}, \mathrm{OCH}_{2} \mathrm{CH}_{2} \mathrm{O}\right), 65.0\left(-, \mathrm{CH}_{2}, \mathrm{OCH}_{2} \mathrm{CH}_{2} \mathrm{O}\right)$, $67.2\left(-, \mathrm{CH}_{2}, \mathrm{CH}_{2} \mathrm{O}\right), 96.8$ (-, $\left.\mathrm{CH}_{2}, \mathrm{OCH}_{2} \mathrm{O}\right), 123.6$ (-, $\left.\mathrm{C}_{\text {quat }}\right), 123.9$ $\left(-, \mathrm{C}_{\text {quat }}\right), 129.9\left(-, \mathrm{C}_{\text {quat }}\right), 131.9\left(-, \mathrm{C}_{\text {quat }}\right) \mathrm{ppm}$. MS $(70 \mathrm{eV}): \mathrm{m} / \mathrm{z}$ $(\%)=266(48)\left[\mathrm{M}^{+}\right], 264(5), 235(5)\left[\mathrm{M}^{+}-\mathrm{CH}_{2} \mathrm{OH}\right], 205$ (14), 204 (24), 191 (100), 179 (6), 176 (7), 163 (10), 162 (8), 149 (68), 133 (5), 120 (8), 117 (6), 105 (10), 91 (28), 77 (18), 67 (5), 65 (9), 55 (12). HRMS: calcd. for $\mathrm{C}_{15} \mathrm{H}_{22} \mathrm{O}_{4} 266.1519$ (correct HRMS).

(E)-3-[2'-(5', $6^{\prime \prime}$-Dihydro[1 ${ }^{\prime \prime}, 4^{\prime \prime}$ ']dioxin-2' '-yl)cyclohex-1' -enyl]prop2-en-1-ol (35): According to GP 4, the hexatriene 20 (274 mg, $0.937 \mathrm{mmol}$ ) in toluene (10.0 mL), DIBALH (4.69 mL, $4.69 \mathrm{mmol})$, workup with diethyl ether $(75 \mathrm{~mL}), \mathrm{KHSO}_{4}$ solution $(20 \mathrm{~mL})$, extraction with diethyl ether $(25 \mathrm{~mL}), \mathrm{NaHCO}_{3}$ solution $(20 \mathrm{~mL})$, water $(20 \mathrm{~mL})$ and FC (24 g of silica gel, 1:1 v/v pentane/diethyl ether elution) yielded compound $35(177 \mathrm{mg}, 85 \%)$ as a colorless 
oil. $R_{\mathrm{f}}=0.3$. IR (Film): $\tilde{v}=3405 \mathrm{~cm}^{-1}, 2928,2870,2837,1651$, 1499, 1450, 1434, 1366, 1306, 1284, 1258, 1235, 1209, 1149, 1091, 1026, 1010, 970, 921, 902, 879. ${ }^{1} \mathrm{H}$ NMR (300 MHz, $\left.\mathrm{CDCl}_{3}\right): \delta=$ 1.49-1.74 [m, $\left.4 \mathrm{H}, 4^{\prime}\left(5^{\prime}\right)-\mathrm{H}\right], 2.14-2.35\left[\mathrm{~m}, 4 \mathrm{H}, 3^{\prime}\left(6^{\prime}\right)-\mathrm{H}\right], 4.05-$ $4.15\left[\mathrm{~m}, 4 \mathrm{H}, 5^{\prime \prime}\left(6^{\prime \prime}\right)-\mathrm{H}\right], 4.20\left(\mathrm{dt},{ }^{3} J_{1}=6.3,{ }^{3} J_{2}=1.1 \mathrm{~Hz}, 2 \mathrm{H}, 1-\right.$ $\mathrm{H}), 5.78\left(\mathrm{dt},{ }^{3} J_{1}=15.9,{ }^{3} J_{2}=6.0 \mathrm{~Hz}, 1 \mathrm{H}, 1-\mathrm{H}\right), 5.92\left(\mathrm{~s}, 1 \mathrm{H}, 3^{\prime \prime}{ }_{-}\right.$ $\mathrm{H}), 6.86\left(\mathrm{~d},{ }^{3} \mathrm{~J}=15.9 \mathrm{~Hz}, 1 \mathrm{H}, 3-\mathrm{H}\right) \mathrm{ppm} .{ }^{13} \mathrm{C} \mathrm{NMR}(75.6 \mathrm{MHz}$, $\mathrm{CDCl}_{3}$, add. APT): $\delta=22.3\left(-, \mathrm{CH}_{2}\right), 22.5\left(-, \mathrm{CH}_{2}\right), 25.9\left(-, \mathrm{CH}_{2}\right)$, $29.0\left(-, \mathrm{CH}_{2}\right), 64.2\left[-, \mathrm{CH}_{2}, 2 \mathrm{C}, \mathrm{C}-5^{\prime \prime}\left(6^{\prime \prime}\right)\right], 64.3\left(-, \mathrm{CH}_{2}, \mathrm{C}-1\right)$, $125.7(+, \mathrm{CH}), 126.5(+, \mathrm{CH}), 131.3\left(-, \mathrm{C}_{\text {quat }}\right), 131.8(+, \mathrm{CH}), 132.3$ $\left(-, \mathrm{C}_{\text {quat }}\right), 136.0\left(-, \mathrm{C}_{\text {quat }}\right) \mathrm{ppm}$. MS $(70 \mathrm{eV}): \mathrm{m} / z(\%)=222(46)$ $\left[\mathrm{M}^{+}\right], 204(7)\left[\mathrm{M}^{+}-\mathrm{H}_{2} \mathrm{O}\right], 191$ (98), 189 (7), $176(8), 164$ (6), 161 (11) 149 (100), 147 (100), 136 (6), 135 (8), 131 (9), 121 (8), 119 (17), 107 (20), 105 (15), 93 (13), 91 (50), 81 (9), 79 (29), 77 (32), 73 (12), 65 (16), 57 (6), 55 (23). HRMS: calcd. for $\mathrm{C}_{13} \mathrm{H}_{18} \mathrm{O}_{3} 222.1257$ (correct HRMS).

$5-\left\{2^{\prime}-\left[(E)-3^{\prime \prime}\right.\right.$-Methoxymethylenoxyprop-1-enyl]cyclohex-1' -enyl $\}$ 2,3-dihydro[1,4]dioxine (36): A solution of the allylic alcohol 35 (180 mg, $0.810 \mathrm{mmol}$ ), ethyldiisopropylamine (523 mg, $4.05 \mathrm{mmol}$ ) and 4-(dimethylamino)pyridine $(39.9 \mathrm{mg}, 0.323 \mathrm{mmol})$ in dichloromethane $(2.00 \mathrm{~mL})$ was treated with methoxymethyl chloride (130 mg, $1.62 \mathrm{mmol}$ ) at $0{ }^{\circ} \mathrm{C}$ and stirred for $1 \mathrm{~h}$. After warming to ambient temperature, stirring was continued for $1 \mathrm{~h}$. The reaction mixture was then poured into diethyl ether $(75 \mathrm{~mL})$ and washed with $1 \mathrm{M} \mathrm{KHSO}_{4}$ solution $(2 \times 15 \mathrm{~mL})$. After extraction of the combined aqueous phases with diethyl ether $(2 \times 25 \mathrm{~mL})$, the combined organic layers were washed with satd. $\mathrm{NaHCO}_{3}(15 \mathrm{~mL})$, water $(15 \mathrm{~mL})$ and dried with $\mathrm{MgSO}_{4}$. Concentration under reduced pressure and FC ( $20 \mathrm{~g}$ of silica, 2:1 v/v pentane/diethyl ether elution) yielded compound $36(166 \mathrm{mg}, 77 \%)$ as a colorless oil. $R_{\mathrm{f}}=0.5$. IR (film): $\tilde{v}=2928 \mathrm{~cm}^{-1}, 2877,1651,1458,1450,1367,1305,1285$, 1212, 1149, 1093, 1042, 1027, 970, 953, 921, 880, 839, 787, 734, 694. ${ }^{1} \mathrm{H}$ NMR (300 MHz, $\left.\mathrm{CDCl}_{3}\right): \delta=1.53-1.75\left[\mathrm{~m}, 4 \mathrm{H}, 4^{\prime}\left(5^{\prime}\right)-\right.$ $\mathrm{H}$ ], 2.13-2.34 [m, $\left.4 \mathrm{H}, 3^{\prime}\left(6^{\prime}\right)-\mathrm{H}\right], 3.38\left(\mathrm{~s}, 3 \mathrm{H}, \mathrm{OCH}_{3}\right), 4.01-4.22$ $\left[\mathrm{m}, 6 \mathrm{H}, 5(6)-\mathrm{H}, 1^{\prime \prime}-\mathrm{H}\right], 4.65\left(\mathrm{~s}, 2 \mathrm{H}, \mathrm{O}-\mathrm{CH}_{2}-\mathrm{O}\right), 5.70\left(\mathrm{dt},{ }^{3} J_{1}=\right.$ $\left.15.7,{ }^{3} J_{2}=6.6 \mathrm{~Hz}, 1 \mathrm{H}, 2^{\prime \prime}-\mathrm{H}\right), 5.92(\mathrm{~s}, 1 \mathrm{H}, 6-\mathrm{H}), 6.87\left(\mathrm{~d},{ }^{3} J=\right.$ $\left.15.7 \mathrm{~Hz}, 1 \mathrm{H}, 3{ }^{\prime \prime}-\mathrm{H}\right) \mathrm{ppm} .{ }^{13} \mathrm{C} \mathrm{NMR}\left(75.6 \mathrm{MHz}, \mathrm{CDCl}_{3}\right.$, add. APT): $\delta=22.3\left(-, \mathrm{CH}_{2}\right), 22.5\left(-, \mathrm{CH}_{2}\right), 25.9\left(-, \mathrm{CH}_{2}\right), 28.9$ (-, $\left.\mathrm{CH}_{2}\right), 55.2\left(+, \mathrm{CH}_{3}, \mathrm{OCH}_{3}\right), 64.2\left(-, \mathrm{CH}_{2}, \mathrm{O}-\mathrm{CH}_{2} \mathrm{CH}_{2} \mathrm{O}\right), 64.3$ (-, $\left.\mathrm{CH}_{2}, \mathrm{O}-\mathrm{CH}_{2} \mathrm{CH}_{2} \mathrm{O}\right), 68.6\left(-, \mathrm{CH}_{2}, \mathrm{C}-1^{\prime \prime}\right), 95.6\left(-, \mathrm{CH}_{2}, \mathrm{O}-\mathrm{CH}_{2-}\right.$ $\mathrm{O}), 122.7(+, \mathrm{CH}), 126.6(+, \mathrm{CH}), 131.3\left(-, \mathrm{C}_{\text {quat }}\right), 132.3\left(-, \mathrm{C}_{\text {quat }}\right)$, $133.2(+, \mathrm{CH}), 135.9\left(-, \mathrm{C}_{\text {quat }}\right)$ ppm. MS $(70 \mathrm{eV}): \mathrm{m} / \mathrm{z}(\%)=266$ (29) $\left[\mathrm{M}^{+}\right], 234$ (14) $\left[\mathrm{M}^{+}-\mathrm{CH}_{3} \mathrm{OH}\right], 221(13)\left[\mathrm{M}^{+}-\mathrm{CH}_{3} \mathrm{OCH}_{2} \mathrm{O}\right]$, 205 (34), 204 (22), 191 (100), 176 (10), 163 (14), 149 (82), 133 (12), 120 (14), 107 (13), 105 (15), 91 (45), 81 (6), 79 (21), 65 (10), 55 (12). HRMS: calcd. for $\mathrm{C}_{15} \mathrm{H}_{22} \mathrm{O}_{4} 266.1519$ (correct HRMS).

tert-Butyl 3-Benzyl-1,2,3,4,4a,5,7,8,9,10-decahydrobenzo[f]isoquinoline-5-carboxylate (37) and tert-Butyl 3-Benzyl-1,2,3,4,5,6,7,8,9,10decahydrobenzo $[f]$ isoquinoline-5-carboxylate (38): According to GP 5, the hexatriene $21(40.0 \mathrm{mg}, 0.105 \mathrm{mmol})$ in decalin $(1.00 \mathrm{~mL})$ was heated at $205^{\circ} \mathrm{C}$ for $1.5 \mathrm{~h}$. After FC ( $20 \mathrm{~g}$ of silica gel, $1: 1 \mathrm{v} / \mathrm{v}$ pentane/diethyl ether elution), a mixture of compounds $\mathbf{3 7}$ and $\mathbf{3 8}$ in a ratio of 1:2.6 (according to ${ }^{1} \mathrm{H}$ NMR) was obtained as a colorless wax (29.1 mg, $73 \%)$. $R_{\mathrm{f}}=0.4$. IR (film): $\tilde{\mathrm{v}}=3086 \mathrm{~cm}^{-1}, 3028$, 2927, 2858, 2833, 1726, 1703, 1684, 1494, 1453, 1391, 1366, 1350, $1283,1253,1211,1149,1085,1076,1064,1000,970,934,910,849$. ${ }^{1} \mathrm{H}$ NMR $\left(300 \mathrm{MHz}, \mathrm{CDCl}_{3}\right.$ signals which can be assigned to the minor product 37 are marked with $\left.{ }^{*}\right): \delta=1.29\left[\mathrm{~s}, 9 \mathrm{H}, \mathrm{CO}_{2} \mathrm{C}\right.$ $\left.\left(\mathrm{CH}_{3}\right)_{3}\right], 1.43-1.64(\mathrm{~m}, 6 \mathrm{H}), 1.81-2.00(\mathrm{~m}, 3 \mathrm{H}), 2.01-2.20(\mathrm{~m}$, $5 \mathrm{H}), 2.22-2.48(\mathrm{~m}, 2 \mathrm{H}), 2.46-2.60(\mathrm{~m}, 3 \mathrm{H}), 2.63-2.74(\mathrm{~m}, 2 \mathrm{H})$, 2.89-3.08 (m, 3 H), 3.31-3.63 (m, $4 \mathrm{H}), 5.39-5.53(\mathrm{~m}, 1 \mathrm{H})^{\#}, 7.05-$ $7.31(\mathrm{~m}, 3 \mathrm{H}, \mathrm{Ph}), 7.33-7.52(\mathrm{~m}, 2 \mathrm{H}, \mathrm{Ph}) \mathrm{ppm} .{ }^{13} \mathrm{C} \mathrm{NMR}$ (75.6 $\mathrm{MHz}, \mathrm{CDCl}_{3}$, add. APT): $\delta=22.8\left(-, \mathrm{CH}_{2}\right), 23.2\left(-, \mathrm{CH}_{2}\right)$, $23.4\left(-, \mathrm{CH}_{2}\right), 23.5\left(-, \mathrm{CH}_{2}\right), 24.8\left(-, \mathrm{CH}_{2}\right), 25.8\left(-, \mathrm{CH}_{2}\right), 25.9$ $\left(-, \mathrm{CH}_{2}\right), 28.1\left[+, 3 \mathrm{C}, \mathrm{CO}_{2} \mathrm{C}\left(\mathrm{CH}_{3}\right)_{3}\right], 28.3\left[+, 3 \mathrm{C}, \mathrm{CO}_{2} \mathrm{C}\left(\mathrm{CH}_{3}\right)_{3}\right]^{\#}$, $29.9\left(-, \mathrm{CH}_{2}\right), 30.3\left(-, \mathrm{CH}_{2}\right), 30.5(+, \mathrm{CH}), 30.7\left(-, \mathrm{CH}_{2}\right), 40.0(+$, $\mathrm{CH}), 43.1(+, \mathrm{CH}), 55.9\left(-, \mathrm{CH}_{2}\right), 56.4\left(-, \mathrm{CH}_{2}\right), 63.2\left(-, \mathrm{CH}_{2}\right)$, $80.4\left[\mathrm{C}_{\text {quat }}, \mathrm{CO}_{2} \mathrm{C}\left(\mathrm{CH}_{3}\right)_{3}\right], 123.2(+, \mathrm{CH})^{\#}, 126.1\left(-, \mathrm{C}_{\text {quat }}\right), 127.2$ $(+, \mathrm{CH}), 127.7(+, \mathrm{CH}), 128.1\left(-, \mathrm{C}_{\text {quat }}\right), 128.5(+, \mathrm{CH}), 129.3(+$, $\mathrm{CH}), 129.4(+, \mathrm{CH}), 129.5(+, \mathrm{CH}), 132.3\left(-, \mathrm{C}_{\text {quat }}\right), 133.6(-$, $\left.\mathrm{C}_{\text {quat }}\right), 137.0\left(-, \mathrm{C}_{\text {quat }}\right), 138.8\left(-, \mathrm{C}_{\text {quat }}\right), 173.7\left(-, \mathrm{C}_{\text {quat }}, \mathrm{C}=\mathrm{O}\right) \mathrm{ppm}$. MS $(70 \mathrm{eV}): m / z(\%)=379(77)\left[\mathrm{M}^{+}\right], 324(22), 323(55)\left[\mathrm{M}^{+}{ }_{-}\right.$ $\mathrm{C}_{4} \mathrm{H}_{8}$ ], 322 (65) [ $\mathrm{M}^{+}-\mathrm{C}_{4} \mathrm{H}_{9}$ ], 306 (34), 278 (52), 276 (22), 232 (31), 204 (39), 186 (13), 159 (30), 146 (20), 134 (66), 120 (89), 117 (23), 115 (15), 105 (13), 91 (100), 77 (8), 65 (15), 57 (42) $\left[\mathrm{C}_{4} \mathrm{H}_{9}{ }^{+}\right]$. HRMS: calcd. for $\mathrm{C}_{25} \mathrm{H}_{33} \mathrm{NO}_{2} 379.2513$ (correct HRMS).

(E)-3-[2'-(1' '-Benzyl-1" $, 2^{\prime \prime}, 3^{\prime \prime}, 6^{\prime \prime}$-tetrahydropyridin-4' '-yl)cyclohex-1'-enyl]prop-2-en-1-ol (39): According to GP 4, the hexatriene $21(90.0 \mathrm{mg}, 0.237 \mathrm{mmol})$ in toluene $(3.00 \mathrm{~mL})$ was treated with DIBALH $(1.90 \mathrm{~mL}, 1.90 \mathrm{mmol})$. The reaction mixture was poured into diethyl ether $(75 \mathrm{~mL})$, the solution washed with satd. $\mathrm{NH}_{4} \mathrm{Cl}$ solution $(2.0 \mathrm{~mL})$ and treated with $1 \mathrm{~N} \mathrm{NaOH}$ solution $(20.0 \mathrm{~mL})$. The resulting precipitate was filtered through Celite. After extraction of the combined aqueous phases with diethyl ether $(2 \times 30 \mathrm{~mL})$, the combined organic layers were dried with $\mathrm{MgSO}_{4}$. Concentration in vacuo and FC of the residue ( $20 \mathrm{~g}$ of silica gel, 10:1 v/v diethyl ether/methanol elution) yielded compound 39 $(65.1 \mathrm{mg}, 89 \%)$ as a yellow oil. $R_{\mathrm{f}}=0.7$. IR (film): $\tilde{v}=3395 \mathrm{~cm}^{-1}$, 3028, 2929, 2798, 1613, 1494, 1452, 1391, 1366, 1310, 1148, 1029, 983, 856, 807, 753. ${ }^{1} \mathrm{H}$ NMR (300 MHz, $\left.\mathrm{CDCl}_{3}\right): \delta=1.50-1.71(\mathrm{~m}$, $3 \mathrm{H}), 2.05-2.20(\mathrm{~m}, 4 \mathrm{H}), 2.51-2.75(\mathrm{~m}, 5 \mathrm{H}), 3.00-3.07(\mathrm{~m}, 2 \mathrm{H}$, $\left.2^{\prime \prime}-\mathrm{H}\right), 3.60$ (s, $\left.2 \mathrm{H}, \mathrm{PhCH}_{2}\right), 4.15\left(\mathrm{~d},{ }^{3} \mathrm{~J}=5.2 \mathrm{~Hz}, 2 \mathrm{H}, 1-\mathrm{H}\right), 5.28-$ $5.34\left(\mathrm{~m}, 1 \mathrm{H}, 3^{\prime \prime}-\mathrm{H}\right), 5.68\left(\mathrm{dt},{ }^{3} J_{1}=15.9,{ }^{3} J_{2}=5.8 \mathrm{~Hz}, 1 \mathrm{H}, 2-\mathrm{H}\right)$, $\left(\mathrm{d},{ }^{3} \mathrm{~J}=15.9 \mathrm{~Hz}, 1 \mathrm{H}, 3-\mathrm{H}\right), 7.23-7.39(\mathrm{~m}, 5 \mathrm{H}, \mathrm{Ph}-\mathrm{H}) \mathrm{ppm} .{ }^{13} \mathrm{C}$ NMR (75.6 MHz, $\mathrm{CDCl}_{3}$, add. APT): $\delta=22.5\left(-, \mathrm{CH}_{2}\right), 25.1(-$, $\left.\mathrm{CH}_{2}\right), 28.5\left(-, \mathrm{CH}_{2}\right), 29.9\left(-, \mathrm{CH}_{2}\right), 49.7\left(-, \mathrm{CH}_{2}\right), 50.4\left(-, \mathrm{CH}_{2}\right)$, $52.5\left(-, \mathrm{CH}_{2}\right), 62.7\left(-, \mathrm{CH}_{2}, \mathrm{PhCH}_{2}\right), 63.6\left(-, \mathrm{CH}_{2}, \mathrm{C}-1\right), 121.7$ (+, $\left.\mathrm{CH}, \mathrm{C}-3^{\prime \prime}\right), 124.8(+, \mathrm{CH}), 127.2(+, \mathrm{CH}, \mathrm{Ph}), 128.0\left(-, \mathrm{C}_{\text {quat }}\right)$, $128.2(+, 2 \mathrm{C}, \mathrm{CH}, \mathrm{Ph}), 129.5(+, 2 \mathrm{C}, \mathrm{CH}, \mathrm{Ph}), 131.0(+, \mathrm{CH})$, 137.0 (-, $\left.\mathrm{C}_{\text {quat }}\right), 137.7$ (-, $\left.\mathrm{C}_{\text {quat }}, \mathrm{Ph}\right), 140.0$ (-, $\left.\mathrm{C}_{\text {quat }}\right)$ ppm. MS $(70 \mathrm{eV}): m / z(\%)=309(42)\left[\mathrm{M}^{+}\right], 308(44), 305(43), 292(23), 278$ (39), 264 (80), 252 (30), 218 (7) 200 (4), 172 (15), 159 (22), 146 (25), 134 (14), 105 (14), 91 (100) [Bn+ ], 77 (11), 65 (16), 63 (3). HRMS: calcd. for $\mathrm{C}_{21} \mathrm{H}_{27} \mathrm{NO} 309.2094$ (correct HRMS).

(3-Benzyl-1,2,3,4,5,6,7,8,9,10-decahydrobenzo[ $f$ |isoquinolin-5-yl)methanol (40) and (3-Benzyl-1,2,3,4,4a,5,7,8,9,10-decahydrobenzo[flisoquinolin-5-yl)methanol (41): According to GP 5, the hexatriene $39(20.0 \mathrm{mg}, 0.0650 \mathrm{mmol})$ in decalin $(0.50 \mathrm{~mL})$ was heated at $205^{\circ} \mathrm{C}$ for $0.5 \mathrm{~h}$. FC (10 g of silica gel, $20: 1 \mathrm{v} / \mathrm{v}$ diethyl ether/methanol elution) gave a mixture of compounds $\mathbf{4 0}$ and $\mathbf{4 1}$ with a ratio of 4.3:1 (according to ${ }^{1} \mathrm{H}$ NMR) as a colorless wax (16.4 mg, 82\%). $R_{\mathrm{f}}=0.6 .{ }^{1} \mathrm{H}$ NMR $\left(300 \mathrm{MHz}, \mathrm{CDCl}_{3}\right.$, signals which can be assigned to the minor component $\mathbf{4 1}$ are marked with $\left.{ }^{\#}\right): \delta=1.05$ 1.29 (m, $6 \mathrm{H}), 1.31-1.72(\mathrm{~m}, 6 \mathrm{H}), 1.98-2.40$ (m, $3 \mathrm{H}), 2.58-2.68$ $\left(\mathrm{m}, 2 \mathrm{H}, \mathrm{PhCH}_{2}\right)^{\#}, 2.60\left(\mathrm{~m}_{\mathrm{c}}, 2 \mathrm{H}, \mathrm{PhCH}_{2}\right), 2.90\left(\mathrm{~m}_{\mathrm{c}}, 1 \mathrm{H}\right)^{\#}, 3.12$ $\left(\mathrm{m}_{\mathrm{c}}, 1 \mathrm{H}\right)^{\#}, 3.26-3.74(\mathrm{~m}, 2 \mathrm{H}), 3.09-3.21(\mathrm{~m}, 2 \mathrm{H}), 3.30-3.54(\mathrm{~m}$, $4 \mathrm{H})$, 5.34-5.38 (m, $1 \mathrm{H}), 6.99-7.31$ (m, 3 H, Ar), 7.32-7.56 (m, 2 $\mathrm{H}$, Ar) ppm. ${ }^{13} \mathrm{C} \mathrm{NMR} \mathrm{(75.6} \mathrm{MHz,} \mathrm{CDCl}_{3}$, add. APT): $\delta=23.0$ $\left(-, \mathrm{CH}_{2}\right)^{\#}, 23.5\left(-, \mathrm{CH}_{2}\right)^{\#}, 24.4\left(-, \mathrm{CH}_{2}\right), 24.5\left(-, \mathrm{CH}_{2}\right), 24.8(-$, $\left.\mathrm{CH}_{2}\right)^{\#}, 25.1\left(-, \mathrm{CH}_{2}\right)^{\#}, 26.0\left(-, \mathrm{CH}_{2}\right), 27.0\left(-, \mathrm{CH}_{2}\right), 30.9(-$, $\left.\mathrm{CH}_{2}\right)^{\#}, 31.0\left(-, \mathrm{CH}_{2}\right)^{\#}, 32.0\left(-, \mathrm{CH}_{2}\right), 39.1(+, \mathrm{CH})^{\#}, 39.3(+, \mathrm{CH})$, $40.0(+, \mathrm{CH}), 50.4\left(-, \mathrm{CH}_{2}\right)^{\#}, 52.2\left(-, \mathrm{CH}_{2}\right), 55.8\left(-, \mathrm{CH}_{2}\right), 56.4$ $\left(-, \mathrm{CH}_{2}\right)^{\#}, 61.2\left(-, \mathrm{CH}_{2}\right), 62.3\left(-, \mathrm{CH}_{2}\right)^{\#}, 62.8\left(-, \mathrm{CH}_{2}\right)^{\#}, 63.3(-$, $\left.\mathrm{CH}_{2}\right), 120.9(+, \mathrm{CH}), 125.6\left(-, \mathrm{C}_{\text {quat }}\right)^{\#}, 125.4\left(-, \mathrm{C}_{\text {quat }}\right), 126.9(-$, $\left.\mathrm{C}_{\text {quat }}\right)^{\#}, 127.2\left(-, \mathrm{C}_{\text {quat }}\right)^{\#}, 127.3(+, \mathrm{CH}, \mathrm{Ar}), 127.3(+, \mathrm{CH}, \mathrm{Ar})^{\#}$, $128.4(+, 2 \mathrm{C}, \mathrm{CH}, \mathrm{Ar})^{\#}, 128.5(+, 2 \mathrm{C}, \mathrm{CH}, \mathrm{Ar}), 129.1(+, 2 \mathrm{C}$, 
$\mathrm{CH}, \mathrm{Ar})^{\#}, 129.1\left(-, \mathrm{C}_{\text {quat }}\right), 129.4(+, 2 \mathrm{C}, \mathrm{CH}, \mathrm{Ar}), 136.3\left(-, \mathrm{C}_{\text {quat }}\right)$, $138.6\left(-, \mathrm{C}_{\text {quat }}\right)^{\#}, 138.8\left(-, \mathrm{C}_{\text {quat }}, \mathrm{Ar}\right), 139.4\left(-, \mathrm{C}_{\text {quat }}\right)^{\#}$ ppm. MS $(70 \mathrm{eV}): m / z(\%)=309(63)\left[\mathrm{M}^{+}\right], 278(52), 276(6), 252(43), 250$ (6), 218 (15), 172 (14), 159 (17), 146 (15), 134 (28), 120 (16), 117 (14), 91 (100), 77 (10), 65 (14). HRMS: calcd. for $\mathrm{C}_{21} \mathrm{H}_{27} \mathrm{NO}$ 309.2094 (correct HRMS).

$(E)-\left(1^{\prime \prime} S, 3 a^{\prime \prime} S, 7 a^{\prime \prime} S\right)-3-[8$ '-(1' - tert-Butoxy-7a' '-methyl$2^{\prime \prime}, 3^{\prime \prime}, 3 a^{\prime \prime}, 6^{\prime \prime}, 7^{\prime \prime}, 7 a^{\prime \prime}$-hexahydro-1' ${ }^{\prime \prime} H$-inden-5' ${ }^{\prime}$-yl)-1', $4^{\prime}$-dioxaspiro[4' $\mathbf{4}^{\prime}$ ']dec-7'-en-7'-yl]prop-2-en-1-ol (43): According to GP 4 , the hexatriene $42(180 \mathrm{mg}, 0.381 \mathrm{mmol})$ in toluene $(3.00 \mathrm{~mL})$, DIBALH (3.05 mL, $3.05 \mathrm{mmol})$, workup with diethyl ether $(35 \mathrm{~mL})$, $\mathrm{KHSO}_{4}$ solution $(10 \mathrm{~mL})$, extraction with diethyl ether $(2 \times 25 \mathrm{~mL}), \mathrm{NaHCO}_{3}$ solution $(20 \mathrm{~mL})$, water $(20 \mathrm{~mL})$ and $\mathrm{FC}$ (24 g of silica gel, 3:2 v/v diethyl ether/pentane elution) yielded compound $43(100 \mathrm{mg}, 65 \%)$ as a colorless oil. $R_{\mathrm{f}}=0.4 .{ }^{1} \mathrm{H} \mathrm{NMR}$ $\left(250 \mathrm{MHz}, \mathrm{C}_{6} \mathrm{D}_{6}\right): \delta=1.05\left(\mathrm{~s}, 3 \mathrm{H}, \mathrm{CH}_{3}\right), 1.15\left[\mathrm{~s}, 9 \mathrm{H}, \mathrm{C}\left(\mathrm{CH}_{3}\right)_{3}\right]$, $1.23-1.40(\mathrm{~m}, 2 \mathrm{H}), 1.42-1.78(\mathrm{~m}, 4 \mathrm{H}), 1.80-2.40(\mathrm{~m}, 8 \mathrm{H}), 2.56-$ $2.75(\mathrm{~m}, 1 \mathrm{H}), 2.98$ (br. s, $1 \mathrm{H}, \mathrm{OH}), 3.36\left(\mathrm{t},{ }^{3} \mathrm{~J}=8.3 \mathrm{~Hz}, 1 \mathrm{H}, 1^{\prime \prime}-\right.$ $\mathrm{H}), 3.43\left(\mathrm{~m}_{\mathrm{c}}, 4 \mathrm{H}, 2^{\prime}-\mathrm{H}, 3^{\prime}-\mathrm{H}\right), 3.71\left(\mathrm{~m}_{\mathrm{c}}, 2 \mathrm{H}, 1-\mathrm{H}\right), 5.44(\mathrm{~s}, 1 \mathrm{H}$, $\left.4^{\prime \prime}-\mathrm{H}\right), 5.83\left(\mathrm{~m}_{\mathrm{c}}, 1 \mathrm{H}, 2-\mathrm{H}\right), 6.90\left(\mathrm{~d},{ }^{3} \mathrm{~J}=16.7 \mathrm{~Hz}, 1 \mathrm{H}, 3-\mathrm{H}\right) \mathrm{ppm}$. ${ }^{13} \mathrm{C}$ NMR $\left(62.9 \mathrm{MHz}, \mathrm{CDCl}_{3}\right.$, add. DEPT): $\delta=11.6\left(+, \mathrm{CH}_{3}\right)$, $25.0\left(-, \mathrm{CH}_{2}\right), 27.3\left(-, \mathrm{CH}_{2}\right), 28.9\left[+, 3 \mathrm{C}, \mathrm{C}\left(\mathrm{CH}_{3}\right)_{3}\right], 29.1\left(-, \mathrm{CH}_{2}\right)$, $32.1\left(-, \mathrm{CH}_{2}\right), 32.3\left(-, \mathrm{CH}_{2}\right), 34.4\left(-, \mathrm{CH}_{2}\right), 42.2\left(\mathrm{C}_{\text {quat }}, \mathrm{C}-7 \mathrm{a}^{\prime \prime}\right)$, $43.8\left(+, \mathrm{CH}, \mathrm{C}-3 \mathrm{a}^{\prime \prime}\right), 62.1\left(-, \mathrm{OCH}_{2} \mathrm{CH}_{2} \mathrm{O}\right), 64.1\left(-, \mathrm{OCH}_{2} \mathrm{CH}_{2} \mathrm{O}\right)$, $69.9\left(-, \mathrm{CH}_{2}, \mathrm{C}-1\right), 72.2\left[\mathrm{C}_{\text {quat }}, \mathrm{C}\left(\mathrm{CH}_{3}\right)_{3}\right], 75.2\left(+, \mathrm{CH}, \mathrm{C}-1^{\prime \prime}\right), 79.6$ $\left[\mathrm{C}_{\text {quat }}, \mathrm{CO}_{2} \mathrm{C}\left(\mathrm{CH}_{3}\right)_{3}\right], 110.7\left(\mathrm{C}_{\text {quat }}, \mathrm{C}^{\prime} 5^{\prime}\right), 126.0(+, \mathrm{CH}), 126.43(+$, $\mathrm{CH}), 126.6(+, \mathrm{CH}), 130.9\left(\mathrm{C}_{\text {quat }}\right), 139.0\left(\mathrm{C}_{\text {quat }}\right), 140.5\left(\mathrm{C}_{\text {quat }}\right) \mathrm{ppm}$.

(13S,14S,17S)-(17-tert-Butoxy-13-methyl-1',3' -spiro[2',3]dioxolan-

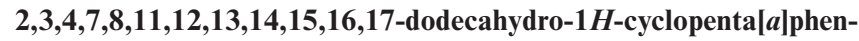
anthren-7-yl)methanol (44) and $(13 S, 14 S, 17 S)-(17-$ tert-Butoxy-13methyl-1' ',3'-spiro[2',3]dioxolan-2,3,4,6,7,11,12,13,14,15,16,17-dodecahydro- $1 H$-cyclopenta[a]phenanthren-7-yl)methanol (45): According to GP 5, the hexatriene $\mathbf{4 3}(80 \mathrm{mg}, 0.20 \mathrm{mmol})$ in decalin $(1.0 \mathrm{~mL})$ was heated at $205^{\circ} \mathrm{C}$ for $0.75 \mathrm{~h}$. FC ( $15 \mathrm{~g}$ of silica gel, $3: 2$ $\mathrm{v} / \mathrm{v}$ diethyl ether/pentane) gave a mixture of compounds 44 and 45 with a ratio of $1: 1.3$ (according to ${ }^{1} \mathrm{H}$ NMR) as a colorless wax $(37 \mathrm{mg}, 46 \%) . R_{\mathrm{f}}=0.4$. IR (film): $\tilde{v}=3396 \mathrm{~cm}^{-1}, 2973,2933,2872$, $1669,1653,1617,1559,1540,1490,1472,1457,1437,1419,1388$, $1362,1253,1193,1108,1062,948,895,812,736,703,668 .{ }^{1} \mathrm{H}$ NMR $\left(300 \mathrm{MHz}, \mathrm{CDCl}_{3}\right.$, signals which can be assigned to the minor component $\mathbf{4 4}$ are marked with $\left.{ }^{\#}\right)$ : $\delta=0.83\left(\mathrm{~s}, 3 \mathrm{H}, \mathrm{CH}_{3}\right)^{\#}$, $0.93\left(\mathrm{~s}, 3 \mathrm{H}, \mathrm{CH}_{3}\right), 1.17$ [s, $\left.9 \mathrm{H}, \mathrm{C}\left(\mathrm{CH}_{3}\right)_{3}\right]^{\#}, 1.18$ [s, $\left.9 \mathrm{H}, \mathrm{C}\left(\mathrm{CH}_{3}\right)_{3}\right]$, $1.21-1.50(\mathrm{~m}, 7 \mathrm{H}), 1.54-2.00(\mathrm{~m}, 8 \mathrm{H}), 2.03-2.52(\mathrm{~m}, 12 \mathrm{H}), 3.20$ $3.38\left(\mathrm{~m}, 4 \mathrm{H}, \mathrm{CH}_{2} \mathrm{OH}\right), 3.41-3.74(\mathrm{~m}, 9 \mathrm{H}), 5.59-5.67(\mathrm{~m}, 1 \mathrm{H}, 6-$ $\mathrm{H})^{\#}$ ppm. MS (70 eV): $m / z(\%)=402(5)\left[\mathrm{M}^{+}\right], 358(4), 342(24)$, 311 (100), 285 (31), 255 (35), 237 (18), 209 (9), 197 (18), 181 (10), 155 (11), 143 (29), $105(5), 91(8), 81(5), 57(64)\left[\mathrm{C}_{4} \mathrm{H}_{9}{ }^{+}\right], 41$ (16).

tert-Butyl trans-1,2,3,4,4a,5,6,7,8,9,10,11,12,12a-Tetradecahydrochrysene-5-carboxylate (48-t $\mathrm{Bu})$ : According to GP 5, the hexatriene 22- $t \mathrm{Bu}(33.2 \mathrm{mg}, 0.110 \mathrm{mmol})$ in decalin $(0.50 \mathrm{~mL})$ was heated at $205^{\circ} \mathrm{C}$ for $45 \mathrm{~min}$. FC (32 g of silica gel, 20:1 v/v light petroleum/ diethyl ether elution) yielded compound $48-t \mathrm{Bu}(23.1 \mathrm{mg}, 77 \%)$ as a colorless oil. $R_{\mathrm{f}}=0.3$. IR (film): $\tilde{v}=2924 \mathrm{~cm}^{-1}, 2853,1711,1448$, $1392,1368,1347,1303,1257,1214,1152,1086,1029,846,736 .{ }^{1} \mathrm{H}$ NMR $\left(250 \mathrm{MHz}, \mathrm{C}_{6} \mathrm{D}_{6}\right): \delta=1.01-1.85(\mathrm{~m}, 18 \mathrm{H}), 1.18[\mathrm{~s}, 9 \mathrm{H}$, $\left.\mathrm{C}\left(\mathrm{CH}_{3}\right)_{3}\right], 1.87-2.08(\mathrm{~m}, 2 \mathrm{H}), 2.08-2.38(\mathrm{~m}, 2 \mathrm{H}), 2.42-2.56(\mathrm{~m}, 2$ $\mathrm{H}, 6-\mathrm{H}), 3.20\left(\mathrm{dd},{ }^{3} J=6.1,{ }^{3} \mathrm{~J}=6.3 \mathrm{~Hz}, 1 \mathrm{H}, 7-\mathrm{H}\right) \mathrm{ppm} .{ }^{13} \mathrm{C} \mathrm{NMR}$ $\left(62.9 \mathrm{MHz}, \mathrm{C}_{6} \mathrm{D}_{6}\right.$, add. DEPT): $\delta=22.9\left(-, \mathrm{CH}_{2}\right), 23.8\left(-, \mathrm{CH}_{2}\right)$, $25.7\left(-, \mathrm{CH}_{2}\right), 26.4\left(-, \mathrm{CH}_{2}\right), 26.9\left(-, \mathrm{CH}_{2}\right), 27.6\left(-, \mathrm{CH}_{2}\right), 28.1[+$, $\left.3 \mathrm{C}, \mathrm{C}\left(\mathrm{CH}_{3}\right)_{3}\right], 30.8\left(-, \mathrm{CH}_{2}\right), 30.9\left(-, \mathrm{CH}_{2}\right), 31.5\left(-, \mathrm{CH}_{2}\right), 32.8$ $\left(-, \mathrm{CH}_{2}\right), 34.5\left(-, \mathrm{CH}_{2}\right), 40.7(+, \mathrm{CH}), 41.4(+, \mathrm{CH}), 44.6(+, \mathrm{CH}$, C-5), $79.2\left[\mathrm{C}_{\text {quat }}, \mathrm{CO}_{2} C\left(\mathrm{CH}_{3}\right)_{3}\right], 128.2\left(\mathrm{C}_{\text {quat }}\right), 129.1\left(\mathrm{C}_{\text {quat }}\right), 131.1$
$\left(\mathrm{C}_{\text {quat }}\right), 173.6\left(\mathrm{C}_{\text {quat }}, \mathrm{C}=\mathrm{O}\right) \mathrm{ppm} \cdot{ }^{13} \mathrm{C} \mathrm{NMR}\left(62.9 \mathrm{MHz}, \mathrm{CDCl}_{3}\right.$, add. DEPT): $\delta=22.5\left(-, \mathrm{CH}_{2}\right), 23.2\left(-, \mathrm{CH}_{2}\right), 25.3\left(-, \mathrm{CH}_{2}\right), 25.9$ $\left(-, \mathrm{CH}_{2}\right), 26.9\left(-, \mathrm{CH}_{2}\right), 27.1\left(-, \mathrm{CH}_{2}\right), 28.0\left[+, \mathrm{C}\left(\mathrm{CH}_{3}\right)_{3}\right], 29.7(-$, $\left.\mathrm{CH}_{2}\right), 30.3\left(-, \mathrm{CH}_{2}\right), 30.8\left(-, \mathrm{CH}_{2}\right), 32.4\left(-, \mathrm{CH}_{2}\right), 34.1\left(-, \mathrm{CH}_{2}\right)$, $40.2(+, \mathrm{CH}), 41.1(+, \mathrm{CH}), 44.2(+, \mathrm{C}-7), 79.2\left[\mathrm{C}_{\text {quat }}, \mathrm{CO}_{2} \mathrm{C}-\right.$ $\left.\left(\mathrm{CH}_{3}\right)_{3}\right], 127.8\left(\mathrm{C}_{\text {quat }}\right), 127.9\left(\mathrm{C}_{\text {quat }}\right), 128.9\left(\mathrm{C}_{\text {quat }}\right), 130.8\left(\mathrm{C}_{\text {quat }}\right)$, $174.3\left(\mathrm{C}_{\text {quat }}, \mathrm{C}=\mathrm{O}\right) \mathrm{ppm}$. MS $(70 \mathrm{eV}): \mathrm{m} / \mathrm{z}(\%)=342(12)\left[\mathrm{M}^{+}\right], 286$ (82) $\left[\mathrm{M}^{+}-\mathrm{C}_{4} \mathrm{H}_{8}\right], 267$ (2), 241 (26), 208 (3), 189 (7), 165 (4), 151 (7), 145 (100), 105 (6), 91 (11), 67 (6), 57 (16) $\left[\mathrm{C}_{4} \mathrm{H}_{9}^{+}\right], 41$ (10). HRMS: calcd. for $\mathrm{C}_{23} \mathrm{H}_{34} \mathrm{O}_{2} 342.2561$ (correct HRMS).

Methyl trans-1,2,3,4,4a,4b,5,6,7,8,9,10,11,12,12a-Tetradecahydrochrysene-5-carboxylate (49): According to GP 5, the hexatriene 22Me $(40.2 \mathrm{mg}, 0.134 \mathrm{mmol})$ in decalin $(0.50 \mathrm{~mL})$ was heated at $205^{\circ} \mathrm{C}$ for $30 \mathrm{~min}$. FC (30 g of neutral aluminum oxide, 20:1 v/v light petroleum/diethyl ether elution) gave compound $49(30.8 \mathrm{mg}$, $77 \%)$ and tetracycle $48-\mathrm{Me}(4.4 \mathrm{mg}, 11 \%)$, as a colorless oil. $R_{\mathrm{f}}=$ 0.3. IR (film): $\tilde{v}=2922 \mathrm{~cm}^{-1}, 2853,1739,1616,1446,1433,1330$, $1272,1259,1232,1190,1165,1112,1095,1070,1023,990,942$, $892,863,851,843,812,765 .{ }^{1} \mathrm{H}$ NMR $\left(250 \mathrm{MHz}, \mathrm{C}_{6} \mathrm{D}_{6}\right): \delta=0.69$ $1.49(\mathrm{~m}, 7 \mathrm{H}), 1.50-1.84(\mathrm{~m}, 7 \mathrm{H}), 1.90-2.26(\mathrm{~m}, 9 \mathrm{H}), 3.02\left(\mathrm{dd},{ }^{3} J\right.$ $\left.=4.5,{ }^{3} \mathrm{~J}=6.1 \mathrm{~Hz}, 1 \mathrm{H}, 5-\mathrm{H}\right), 3.31\left(\mathrm{~s}, 3 \mathrm{H}, \mathrm{CH}_{3}\right), 5.73\left(\mathrm{~m}_{\mathrm{c}}, 1 \mathrm{H}\right.$, 6-H) ppm. ${ }^{13} \mathrm{C}$ NMR $\left(62.9 \mathrm{MHz}, \mathrm{C}_{6} \mathrm{D}_{6}\right.$, add. DEPT): $\delta=23.0(-$, $\left.\mathrm{CH}_{2}\right), 23.5\left(-, \mathrm{CH}_{2}\right), 25.2\left(-, \mathrm{CH}_{2}\right), 26.7\left(-, \mathrm{CH}_{2}\right), 26.8\left(-, \mathrm{CH}_{2}\right)$, $31.2\left(-, \mathrm{CH}_{2}\right), 31.6\left(-, \mathrm{CH}_{2}\right), 34.0\left(-, \mathrm{CH}_{2}\right), 34.3\left(-, \mathrm{CH}_{2}\right), 35.3$ $\left(-, \mathrm{CH}_{2}\right), 38.5(+, \mathrm{CH}), 39.3(+, \mathrm{CH}), 41.3(+, \mathrm{CH}), 45.1(+, \mathrm{C}-5)$, $51.2\left(+, \mathrm{CH}_{3}\right), 118.5(+, \mathrm{C}-6), 127.8\left(\mathrm{C}_{\text {quat }}\right), 128.8\left(\mathrm{C}_{\text {quat }}\right), 135.3$ $\left(\mathrm{C}_{\text {quat }}\right), 173.9\left(\mathrm{C}_{\text {quat }}, \mathrm{C}=\mathrm{O}\right) \mathrm{ppm}$. MS $(70 \mathrm{eV}): \mathrm{m} / \mathrm{z}(\%)=300(100)$ $\left[\mathrm{M}^{+}\right], 296$ (7), 266 (21) [M+ $\left.-\mathrm{CO}_{2} \mathrm{CH}_{3}\right], 240$ (69), 238 (18), 212 (10), 197 (12), 183 (7), 171 (9), 145 (73), 141 (12), 91 (6), 79 (4), 44 (76), 41 (3). HRMS: calcd. for $\mathrm{C}_{20} \mathrm{H}_{28} \mathrm{O}_{2} 300.2091$ (correct HRMS).

Methyl trans-1,2,3,4,4a,5,6,7,8,9,10,11,12,12a-Tetradecahydrochrysene-5-carboxylate (48-Me): According to GP 5, the hexatriene 22Me $(280 \mathrm{mg}, 0.933 \mathrm{mmol})$ in decalin $(5.00 \mathrm{~mL})$ was heated at $205^{\circ} \mathrm{C}$ for $45 \mathrm{~min}$. FC ( $28 \mathrm{~g}$ of silica gel, $20: 1 \mathrm{v} / \mathrm{v}$ light petroleum/ diethyl ether elution) gave compound 48-Me (193 mg, 69\%) as a colorless wax. $R_{\mathrm{f}}=0.3$. IR (film): $\tilde{v}=2925 \mathrm{~cm}^{-1}, 2853,1728,1595$, $1447,1331,1275,1195,1072,1021,1001,969,911,859,735 .{ }^{1} \mathrm{H}$ NMR $\left(250 \mathrm{MHz}, \mathrm{CDCl}_{3}\right): \delta=0.82-1.89(\mathrm{~m}, 16 \mathrm{H}), 1.91-2.18(\mathrm{~m}$, $6 \mathrm{H}), 2.20-2.43(\mathrm{~m}, 2 \mathrm{H}), 3.21\left(\mathrm{dd},{ }^{3} J=7.6,{ }^{3} \mathrm{~J}=9.1 \mathrm{~Hz}, 1 \mathrm{H}, 5-\right.$ $\mathrm{H}), 3.62\left(\mathrm{~s}, 3 \mathrm{H}, \mathrm{CH}_{3}\right) \mathrm{ppm} .{ }^{13} \mathrm{C} \mathrm{NMR}\left(62.9 \mathrm{MHz}, \mathrm{CDCl}_{3}\right.$, add. DEPT): $\delta=22.4\left(-, \mathrm{CH}_{2}\right), 22.6\left(-, \mathrm{CH}_{2}\right), 23.1\left(-, \mathrm{CH}_{2}\right), 25.3(-$, $\left.\mathrm{CH}_{2}\right), 25.9\left(-, \mathrm{CH}_{2}\right), 26.4\left(-, \mathrm{CH}_{2}\right), 30.2\left(-, \mathrm{CH}_{2}\right), 30.4\left(-, \mathrm{CH}_{2}\right)$, $31.2\left(-, \mathrm{CH}_{2}\right), 32.0\left(-, \mathrm{CH}_{2}\right), 33.3\left(-, \mathrm{CH}_{2}\right), 38.7(+, \mathrm{CH}), 40.9(+$, $\mathrm{CH}), 43.8(+, \mathrm{CH}, \mathrm{C}-5), 53.0\left(+, \mathrm{CH}_{3}\right), 127.8\left(\mathrm{C}_{\text {quat }}\right), 127.8\left(\mathrm{C}_{\text {quat }}\right)$, $128.0\left(\mathrm{C}_{\text {quat }}\right), 131.3\left(\mathrm{C}_{\text {quat }}\right), 175.3\left(\mathrm{C}_{\text {quat }}, \mathrm{C}=\mathrm{O}\right) \mathrm{ppm}$. MS $(70 \mathrm{eV})$ : $m / z(\%)=300(52)\left[\mathrm{M}^{+}\right], 266(2)\left[\mathrm{M}^{+}-\mathrm{CO}_{2} \mathrm{CH}_{3}\right], 241$ (12), 226 (10), 218 (2), 192 (11), 190 (10), 162 (4), 146 (14), 145 (100), 105 (14), 239 (5), 197 (9), 171 (8), 145 (100), 129 (8), 117 (5), 91 (6), 79 (3), 67 (4), 41 (4). HRMS: calcd. for $\mathrm{C}_{20} \mathrm{H}_{28} \mathrm{O}_{2} 300.2091$ (correct HRMS).

tert-Butyl 13-tert-Butoxy-12a-methyl-1,2,3,4,4a,4b,5,6, 7,8,9,10,11,12, 12a-tetradecahydrochrysene-5-carboxylate (50): According to GP 5, the hexatriene 23 (100 mg, $0.234 \mathrm{mmol})$ in decalin $\left(3.00 \mathrm{~mL}\right.$ ) was heated at $205^{\circ} \mathrm{C}$ for $45 \mathrm{~min}$. FC ( $33 \mathrm{~g}$ of silica gel, $20: 1 \mathrm{v} / \mathrm{v}$ light petroleum/diethyl ether elution) gave compound $\mathbf{5 0}$ (71.4 mg, $71 \%$ ) as a colorless wax. $R_{\mathrm{f}}=0.3$. IR (film): $\tilde{\mathrm{v}}=$ $2974 \mathrm{~cm}^{-1}, 2927,2854,1725,1595,1450,1388,1378,1368,1332$, 1284, 1270, 1249, 1227, 1192, 1154, 1067, 1023, 1000, 970, 957, 945, 903, 884, 852, 820, 759, 703. ${ }^{1} \mathrm{H}$ NMR $\left(250 \mathrm{MHz}, \mathrm{CDCl}_{3}\right): \delta$ $=0.78\left(\mathrm{~s}, 3 \mathrm{H}, \mathrm{CH}_{3}\right), 1.18\left[\mathrm{~s}, 9 \mathrm{H}, \mathrm{C}\left(\mathrm{CH}_{3}\right)_{3}\right], 1.39[\mathrm{~s}, 9 \mathrm{H}$, $\left.\mathrm{CO}_{2} \mathrm{C}\left(\mathrm{CH}_{3}\right)_{3}\right], 1.43-1.62(\mathrm{~m}, 6 \mathrm{H}), 1.64-1.85(\mathrm{~m}, 7 \mathrm{H}), 1.86-2.10$ 
(m, 5 H), 2.13-2.36 (m, 3 H), 2.91-3.00 (m, 1 H, 5-H), 3.09 (dd, ${ }^{3} J$ $\left.=5.6,{ }^{3} \mathrm{~J}=11.4 \mathrm{~Hz}, 1 \mathrm{H}, 1-\mathrm{H}\right) \mathrm{ppm} .{ }^{13} \mathrm{C} \mathrm{NMR}\left(62.9 \mathrm{MHz}, \mathrm{C}_{6} \mathrm{D}_{6}\right.$, add. DEPT): $\delta=11.3\left(+, \mathrm{CH}_{3}\right), 22.9\left(-, \mathrm{CH}_{2}\right), 23.0\left(-, \mathrm{CH}_{2}\right), 23.8$ $\left(-, \mathrm{CH}_{2}\right), 24.9\left(-, \mathrm{CH}_{2}\right), 25.1\left(-, \mathrm{CH}_{2}\right), 28.1\left[+, 3 \mathrm{C}, \mathrm{C}\left(\mathrm{CH}_{3}\right)_{3}\right], 29.3$ [+, $\left.3 \mathrm{C}, \mathrm{CO}_{2} \mathrm{C}\left(\mathrm{CH}_{3}\right)_{3}\right], 30.5\left(-, \mathrm{CH}_{2}\right), 31.0\left(-, \mathrm{CH}_{2}\right), 32.2\left(-, \mathrm{CH}_{2}\right)$, $34.8\left(-, \mathrm{CH}_{2}\right), 38.0(+, \mathrm{CH}, \mathrm{C}-4 \mathrm{a}), 40.0\left(\mathrm{C}_{\text {quat }}, \mathrm{C}-12 \mathrm{a}\right), 46.1(+, \mathrm{C}-$ 5), $72.6\left[\mathrm{C}_{\text {quat }}, C\left(\mathrm{CH}_{3}\right)_{3}\right], 78.3(+, \mathrm{C}-1), 79.1\left[\mathrm{C}_{\text {quat }}, \mathrm{CO}_{2} \mathrm{C}\left(\mathrm{CH}_{3}\right)_{3}\right]$, $127.2\left(\mathrm{C}_{\text {quat }}\right), 127.8\left(\mathrm{C}_{\text {quat }}\right), 128.1\left(\mathrm{C}_{\text {quat }}\right), 130.1\left(\mathrm{C}_{\text {quat }}\right), 172.7$ $\left(\mathrm{C}_{\text {quat }}, \mathrm{C}=\mathrm{O}\right) \mathrm{ppm} .{ }^{13} \mathrm{C} \mathrm{NMR}\left(62.9 \mathrm{MHz}, \mathrm{CDCl}_{3}\right.$, add. DEPT): $\delta$ $=11.3\left(+, \mathrm{CH}_{3}\right), 22.8\left(-, \mathrm{CH}_{2}\right), 23.9\left(-, \mathrm{CH}_{2}\right), 24.8\left(-, \mathrm{CH}_{2}\right), 25.1$ $\left(-, \mathrm{CH}_{2}\right), 28.2\left[+, 3 \mathrm{C}, \mathrm{C}\left(\mathrm{CH}_{3}\right)_{3}\right], 29.4\left[+, 3 \mathrm{C}, \mathrm{CO}_{2} \mathrm{C}\left(\mathrm{CH}_{3}\right)_{3}\right], 30.5$ $\left(-, \mathrm{CH}_{2}\right), 31.1\left(-, \mathrm{CH}_{2}\right), 32.3\left(-, \mathrm{CH}_{2}\right), 34.6\left(-, \mathrm{CH}_{2}\right), 37.8(+, \mathrm{C}-$ 4a), $39.9\left(\mathrm{C}_{\text {quat }}, \mathrm{C}-12 \mathrm{a}\right), 46.0(+, \mathrm{C}-5), 72.7\left[\mathrm{C}_{\text {quat }}, \mathrm{C}\left(\mathrm{CH}_{3}\right)_{3}\right], 78.5$ $(+, \mathrm{C}-1), 126.2\left(\mathrm{C}_{\text {quat }}\right), 128.1\left(\mathrm{C}_{\text {quat }}\right), 128.2\left(\mathrm{C}_{\text {quat }}\right), 130.0\left(\mathrm{C}_{\text {quat }}\right)$, $173.9\left(\mathrm{C}_{\text {quat }}, \mathrm{C}=\mathrm{O}\right) \mathrm{ppm}$. MS $(70 \mathrm{eV}): \mathrm{m} / z(\%)=428(9)\left[\mathrm{M}^{+}\right], 402$ (11), 386 (19), 346 (18), 329 (34), 313 (52), 295 (100), 267 (29), 229 (43), 215 (14), 197 (9), 145 (7), 95 (4), 84 (10), 57 (84) $\left[\mathrm{C}_{4} \mathrm{H}_{9}{ }^{+}\right], 41$ (18). HRMS: calcd. for $\mathrm{C}_{28} \mathrm{H}_{44} \mathrm{O}_{3} 428.3293$ (correct HRMS).

Supporting Information (see also the footnote on the first page of this article): Copies of ${ }^{1} \mathrm{H}$ and ${ }^{13} \mathrm{C}$ NMR spectra of the described compounds.

\section{Acknowledgments}

This work was supported by the State of Niedersachsen as well as the companies BASF AG, Schering AG and Chemetall GmbH (Chemicals). H. W. S. is indebted to the German Merit Foundation (Studienstiftung des deutschen Volkes) for a graduate student fellowship. The authors are grateful to Stefan Beußhausen (Göttingen) for his technical support.

[1] See: a) E. J. Corey, Angew. Chem. 2002, 114, 1724-1741; Angew. Chem. Int. Ed. 2002, 41, 1650-1667 and references cited therein; b) D. A. Evans, J. S. Johnson in Comprehensive Asymmetric Catalysis I-III, vol. 3 (Eds.: E. N. Jacobson, A. Pfaltz, H. Yamamoto), Springer, Berlin 1999, pp. 1177-1235 and references cited therein.

[2] a) J. E. Baldwin in Pericyclic Reactions (Eds.: A. P. Marchand, R. E. Lehr), Academic Press, New York, 1977, vol. II, pp. 273302; b) E. N. Marvell in Thermal Electrocyclic Reactions (Ed.: H. H. Wasserman), Academic Press, New York, 1980, vol. 43, pp. 260-375; c) T. L. Gilchrist, R. J. Summersell, J. Chem. Soc. Perkin Trans. 1 1988, 2595-2601.

[3] For reviews, see: a) S. A. Tucker, J. M. Griffin, W. E. Acree Jr, J. C. Fetzer, M. Zander, O. Reiser, A. de Meijere, I. Murata, Polycyclic Aromat. Compd. 1994, 4, 141-160; b) A. de Meijere, P. von Zezschwitz, H. Nüske, B. Stulgies, J. Organomet. Chem. 2002, 653, 129-140; c) A. de Meijere, P. von Zezschwitz, S. Bräse, Acc. Chem. Res. 2005, 38, 413-422; d) P. von Zezschwitz, A. de Meijere, Top. Organomet. Chem. 2006, 19, 49-89.

[4] a) Metal-Catalyzed Cross-Coupling Reactions, 2nd completely revised ed. (Eds.: A. de Meijere, F. Diederich), Wiley-VCH, Weinheim, 2004; b) Handbook of Palladium Chemistry for Organic Synthesis (Eds.: E. Negishi, A. de Meijere), Wiley, New York, 2002.

[5] a) A. Lansky, O. Reiser, A. de Meijere, Synlett 1990, 405-407; b) O. Reiser, B. König, K. Meerholz, J. Heinze, T. Wellauer, F. Gerson, R. Frim, M. Rabinovitz, A. de Meijere, J. Am. Chem. Soc. 1993, 115, 3511-3518; c) R. von Essen, P. von Zezschwitz, D. Vidović, A. de Meijere, Chem. Eur. J. 2004, 10, 4341-4352.

[6] a) K. Voigt, P. von Zezschwitz, K. Rosauer, A. Lansky, A. Adams, O. Reiser, A. de Meijere, Eur. J. Org. Chem. 1998, 15211534; b) P. von Zezschwitz, F. Petry, A. de Meijere, Chem. Eur. J. 2001, 7, 4035-4046; c) H. W. Sünnemann, A. Hofmeister, J. Magull, A. de Meijere, Chem. Eur. J. 2007, 13, 3739-3756.
[7] a) H. W. Sünnemann, A. de Meijere, Angew. Chem. 2004, 116, 913-915; Angew. Chem. Int. Ed. 2004, 43, 895-897; b) H. W. Sünnemann, A. Hofmeister, J. Magull, A. de Meijere, Chem. Eur. J. 2006, 12, 8336-8344.

[8] a) K. Kikukawa, H. Umekawa, F. Wada, T. Matsuda, Chem. Lett. 1988, 881-884; b) H. X. Zhang, F. Guibe, G. Balavoine, J. Org. Chem. 1990, 55, 1857-1867; c) J. P. Marino, H. N. Nguyen, J. Org. Chem. 2002, 67, 6841-6844.

[9] (tert-Butyldimethylsilyl)acetylene was prepared conveniently by adding $n \mathrm{BuLi}$ to a saturated solution of acetylene in THF and trapping the resulting carbanion with $t \mathrm{BuMeSiCl}$.

[10] a) A. G. Martínez, A. G. Fraile, J. M. S. García, Chem. Ber. 1983, 116, 815-818; b) C. J. Collins, A. G. Martínez, R. M. Alvarez, J. A. Aguirre, Chem. Ber. 1984, 117, 2815-2824; c) M. A. Tius, G. S. K. Kannangara, J. Org. Chem. 1990, 55, 5711-5714; d) M. Mori, N. Kaneta, M. Shibasaki, J. Org. Chem. 1991, 56, 3486-3493.

[11] S. R. Gilbertson, C. A. Challener, M. E. Bos, W. D. Wulff, Tetrahedron Lett. 1988, 29, 4795-4798.

[12] J. E. Mc Murry, W. J. Scott, Tetrahedron Lett. 1983, 24, 979982.

[13] R. K. Summerbell, L. N. Bauer, J. Am. Chem. Soc. 1935, 57, 2364-2368.

[14] For reviews on the Stille reaction, see: a) T. N. Mitchell in Metal-Catalyzed Cross-Coupling Reactions (Eds.: A. de Meijere, F. Diederich), Wiley-VCH, Weinheim 2004, pp. 125-161; b) V. Farina, V. Krishnamurthy, W. J. Scott, The Stille Reaction in Organic Reactions, vol. 50 (Ed.: L. A. Paquette), Wiley, New York, 1997; c) T. N. Mitchell, Synthesis 1992, 803-815; d) M. Kosugi, K. Fugami in Handbook of Organopalladium Chemistry for Organic Synthesis (Eds.: E. Negishi, A. de Meijere), Wiley, New York 2002, pp. 263-284.

[15] For the investigated Stille reactions with enol triflates, $\mathrm{LiCl}$ was an essential additive. Without addition of $\mathrm{LiCl}$, a rapid decomposition of the catalyst was observed. See: W. J. Scott, J. K. Stille, J. Am. Chem. Soc. 1986, 108, 3033-3040.

[16] We thank Dr. A. Edwards for carrying out the single-crystal $\mathrm{X}$-ray analysis of compound 19. Details will be reported elsewhere.

[17] The palladacycle was prepared from $\mathrm{Pd}(\mathrm{OAc})_{2}$ and $(o \mathrm{Tol})_{3} \mathrm{P}$ : a) W. A. Herrmann, C. Brossmer, K. Öfele, C.-P. Reisinger, T. Priermeier, M. Beller, H. Fischer, Angew. Chem. 1995, 107, 1989-1992; Angew. Chem. Int. Ed. Engl. 1995, 34, 1844-1848; b) W. A. Herrmann, C. Brossmer, C.-P. Reisinger, T. Riermeier, K. Öfele, M. Beller, Chem. Eur. J. 1997, 3, 1357-1364; for reviews on the application of palladacycles in Heck couplings, see: c) J. Dupont, C. S. Consorti, J. Spencer, Chem. Rev. 2005, 105, 2527-2571; d) W. A. Herrmann, V. P. W. Böhm, C.-P. Reisinger, J. Organomet. Chem. 1999, 576, 23-41; e) V. Farina, $A d v$. Synth. Catal. 2004, 346, 1553-1582.

[18] Recent results have shown that by adding $\mathrm{P}(o \mathrm{Tol})_{3}$ or dppb as co-ligand to the palladacycle, the catalyst load can be significantly reduced, even providing the hexatrienes in slightly increased yields. See refs. ${ }^{[6 c, 7 b]}$

[19] V. Farina, S. Kapadia, B. Krishnan, C. Wang, L. S. Liebeskind, J. Org. Chem. 1994, 59, 5905-5911.

[20] Unsubstituted ethene and electron-rich alkenes such as enol ethers and vinylsilanes gave only low yields $(<20 \%)$ or complex mixtures in Heck reactions with bromobutadiene $\mathbf{1 7}$ catalyzed by the palladacycle.

[21] Z. Hong, X. Chen, X. Xu, Tetrahedron Lett. 2003, 44, 485488.

[22] a) B. M. Trost, W. Tang, Angew. Chem. 2002, 114, 2919-2921; Angew. Chem. Int. Ed. 2002, 41, 2795-2797; b) B. M. Trost, F. D. Toste, J. Am. Chem. Soc. 2000, 122, 11262-11263; c) A. G. Myers, D. Tanaka, M. R. Mannion, J. Am. Chem. Soc. 2002, 124, 11250-11251; d) H. Bienayme, C. Yezeguelian, Tetrahedron 1994, 50, 3389-3396.

[23] J. D. Connolly, R. A. Hill, Dictionary of Terpenoids, Chapman \& Hall, London, 1991. 
[24] a) B. M. Trost, P. G. McDougal, J. Org. Chem. 1984, 49, 458468; this rotational selectivity has also been termed torquoselectivity; see: b) E. A. Kallel, Y. Wang, D. C. Spellmeyer, K. N. Houk, J. Am. Chem. Soc. 1990, 112, 6759-6763; c) J. D. Evanseck, B. E. Thomas IV, D. C. Spellmeyer, K. N. Houk, J. Org. Chem. 1995, 60, 7134-7141.

[25] a) J. Gutzwiller, P. Buchschacher, A. Fürst, Synthesis 1977, 167-168; b) R. A. Micheli, Z. G. Hajos, N. Cohen, D. R. Parrish, L. A. Portland, W. Sciamanna, M. A. Scott, P. A. Wehrli, J. Org. Chem. 1975, 40, 675-681; c) L. F. Tietze, J. Utecht, Synthesis 1993, 957-958.

[26] Diels-Alder reactions exploiting similar bicyclo[4.4.0]decadienes yielded interesting complex carbon skeletons: R. von Essen, D. Frank, H. W. Sünnemann, D. Vidović, J. Magull, A. de Meijere, Chem. Eur. J. 2005, 11, 6583-6592.

[27] G. Stork, A. Brizzolara, H. Landesman, J. Szmuskovicz, R. Terrell, J. Am. Chem. Soc. 1963, 85, 207-222.

[28] J. B. Hendrickson, R. Bergeron, Tetrahedron Lett. 1973, 14, 4607-4610.

[29] T. Schubert, W. Hummel, M.-R. Kula, M. Müller, Eur. J. Org. Chem. 2001, 4181-4187.

Received: March 6, 2007

Published Online: June 18, 2007 